\title{
DISCONTINUOUS GALERKIN METHODS FOR THE MULTI-DIMENSIONAL VLASOV-POISSON PROBLEM
}

\author{
BLANCA AYUSO DE DIOS, JOSÉ A. CARRILLO, AND CHI-WANG SHU
}

\begin{abstract}
We introduce and analyze two new semi-discrete numerical methods for the multi-dimensional Vlasov-Poisson system. The schemes are constructed by combing a discontinuous Galerkin approximation to the Vlasov equation together with a mixed finite element method for the Poisson problem. We show optimal error estimates in the case of smooth compactly supported initial data. We propose a scheme that preserves the total energy of the system.
\end{abstract}

\section{INTRODUCTION}

The Vlasov-Poisson (VP) system is a classical model in collisionless kinetic theory. It is a mean-field limit description of a large ensemble of interacting particles by electrostatic or gravitational forces. While most of the results in this work are equally valid in both cases under smoothness assumptions of the solutions, we focus our presentation on the plasma physics case.

In kinetic theory, the evolution of the particle number density or mass density $f(\mathbf{x}, \mathbf{v}, t)$ in phase space, i.e. position and velocity $(\mathbf{x}, \mathbf{v})$ at time $t>0$ is given by the Vlasov equation

$$
\frac{\partial f}{\partial t}+\mathbf{v} \cdot \nabla_{\mathbf{x}} f-\nabla_{\mathbf{x}} \Phi \cdot \nabla_{\mathbf{v}} f=0, \quad(\mathbf{x}, \mathbf{v}, t) \in \Omega_{x} \times \mathbb{R}^{d} \times[0, T],
$$

considered with periodic boundary conditions in the $d$-dimensional torus $\Omega_{x}=$ $[0,1]^{d}$ with $d=2,3$. In order to describe charged particles motion in plasmas, we need to compute the force field from the macroscopic density of particles

$$
\rho(\mathbf{x}, t)=\int_{\mathbb{R}^{d}} f(\mathbf{x}, \mathbf{v}, t) d \mathbf{v} .
$$

While in a more accurate model, magnetic effects and Maxwell's equation for the force fields should be considered, we assume that they are negligible and compute the force field from the Poisson equation,

$$
-\Delta \Phi=\rho(\mathbf{x}, t)-1, \quad(\mathbf{x}, t) \in \Omega_{x} \times[0, T],
$$

where $\mathbf{E}(\mathbf{x}, t)=\nabla_{\mathbf{x}} \Phi$ is the electrostatic field per unit mass, up to a sign, acting on particles. Here, we set all physical constants appearing in the equations to one for simplicity. Its solution allows to compute the electric potential $\Phi(\mathbf{x}, t)$ due to both the self-consistent part coming from the macroscopic density $\rho(\mathbf{x}, t)$ and a

2000 Mathematics Subject Classification. 65N30, 65M60, 65M12, 65M15, 82D10. 
uniform background ion density normalized to one. In plasma applications the system has to be globally neutral, meaning that the total charge of the system is zero,

$$
\int_{\Omega_{x}} \rho(\mathbf{x}, t) d \mathbf{x}=\int_{\Omega_{x}} \int_{\mathbb{R}^{d}} f(\mathbf{x}, \mathbf{v}, t) d \mathbf{v} d \mathbf{x}=1
$$

This is a compatibility condition imposed by the periodicity of the boundary conditions. We refer to the surveys $[30,10,26]$ for good account on the state of the art in the mathematical analysis and properties of the solutions of the Cauchy problem for the Vlasov-Poisson system. Global classical solutions were constructed in [9] for the system (1)-(3) with periodic in space boundary conditions and with compactly supported in velocity $C^{2}\left(\Omega_{x} \times \mathbb{R}^{d}\right)$-initial data. Since the solutions are shown to remain compactly supported in velocity if initially so, we will assume without loss of generality that there exists $L>0$ such that $v \in \Omega_{v}=[-L, L]^{d}$ and that

$$
\operatorname{supp}_{(\mathbf{x}, \mathbf{v})}(f(\cdot, t)) \subset \Omega_{x} \times(-L, L)^{d}
$$

for all $0 \leq t \leq T$ for a given fixed $T>0$. The VP system is a infinite dimensional hamiltonian which has infinitely many conserved quantities, in particular all $L^{p}$-norms, $1 \leq p \leq \infty$ of the distribution function and the total (kinetic + potential) energy are preserved in time.

Due to the large number of physical applications and technological implications of the behavior of plasmas, the numerical simulation of the VP system has attracted lots of attention in the last decades since kinetic descriptions are more accurate. Due to the high dimensionality of the system, most of the first attempts were based on particle-like or stochastic methods. Nowadays, there is a strong interest in the design and understanding of accurate deterministic solvers. We will not further discuss about the previous work in numerical methods for the Vlasov-Poisson system and refer the interested author to the introduction of our companion paper [6] or the technical report [7] in the one dimensional case.

This work is the natural extension of [6] where we have proposed and analyzed a wide family of Discontinuous Galerkin schemes for the one-dimensional Vlasov-Poisson system. In this paper we pursue our study with the construction and analysis of numerical schemes to the multi-dimensional case, $d=2,3$.

Following [6], we construct two eulerian schemes, based on the coupling of a DG approximation for the Vlasov equation (1) and mixed finite element approximation for the Poisson problem. In particular we consider the Raviart-Thomas mixed approximation and the Local Discontinuous Galerkin (LDG) method. As we noted in [6] most works in the literature, consider schemes either in primal formulation to approximate the Poisson problem, or direct discretization of the closed form solution of the electrostatic field $E$. This last approach cannot be carried in two or more dimensions. Also since it is the electrostatic field $\boldsymbol{E}$ 
(and not $\Phi$ ) defines the transport in $\boldsymbol{v}$ in (1), we think that a mixed method is more appropriate.

We present the $L^{2}$-error analysis of the proposed methods, in the case of smooth compactly supported solutions. We show optimal error estimates for both the distribution function and the Electrostatic field in the $L^{2}$-norm. To avoid the loss of half order, typical of classical error analyses of hyperbolic problems, we introduce some special projections, inspired mainly in [33], that exploit the structure of the mesh and extend to higher dimension the ones introduced in [6].

It is worth noticing that, although it is a non-linear problem, our error analysis does not require any a-priori assumption on the approximation to the distribution function or the electrostatic field, as it usually happens in the analysis of numerical methods for non-linear problems. As a consequence the error bounds proved are not asymptotic; i.e., hold for any $h<1$. We deal with the nonlinearity, by proving $L^{\infty}$ bounds on the approximate electrostatic field. We wish to mention that the proof of this result (for both the LDG and Raviart-Thomas mixed methods) it is of independent interest. Although there is a large amount of work in the literature, devoted to the $L^{\infty}$ and pointwise error analysis for the approximation of a "linear" Poisson problem (see [28] and [19]), the case where the forcing term in the Poisson problem depends itself of the solution, has not been treated before to the best of our knowledge, for mixed and DG approximations. Our analysis follow the ideas of $[38,37]$, where the authors deal with the conforming approximation of a "general" Poisson problem taking into account the outside influence of the forcing term. However since [38, 37] deals with standard conforming approximation, many of the results and arguments used by the authors in these works cannot be straightforwardly adapted, specially for the LDG method. For the case of the Raviart-Thomas approximation, the seminal work [28] can be more easily adapted to cover the present situation.

One of the motivations for using DG approximation for the Vlasov equation, is that it allows by construction the conservation of mass. In this paper, we also introduce a DG-LDG method for Vlasov-Poisson that preserves the total energy of the system, extending to higher dimensions, the scheme proposed in [6].

We would like to emphasize that to the best of our knowledge, this is the first work providing the error analysis for an eulerian scheme to approximate the Vlasov-Poisson system in dimension $d=2,3$.

The outline of the paper is as follows. In $\S 2$ we present the basic notations we need for the description and analysis of the numerical methods. We also revise some well known results that will be used in the paper. In $\S 3$ we introduce our numerical methods for approximating the Vlasov-Poisson system and show Stability of the proposed schemes. The error analysis is carried out in $\S 4$. The issue of energy conservation is discussed in $\S 5$. The paper is completed with two Appendixes: Appendix A contains the proofs of the error estimates for the electrostatic field; and in Appendix B are included the proofs of some auxiliary results required by our convergence analysis. 


\section{Preliminaries and Basic Notation}

In this section we review the basic notation for the discrete setting and the definition of the finite element spaces. We close the section by reviewing some standard tools of FE methods that will be used in the paper.

Throughout the paper, we use the standard notation for Sobolev spaces [2]. For a bounded domain $B \subset \mathbb{R}^{2}$, we denote by $H^{m}(B)$ the $L^{2}$-Sobolev space of order $m \geq 0$ and by $\|\cdot\|_{m, B}$ and $|\cdot|_{m, B}$ the usual Sobolev norm and seminorm, respectively. For $m=0$, we write $L^{2}(B)$ instead of $H^{0}(B)$. We shall denote by $H^{m}(B) / \mathbb{R}$ the quotient space consisting of equivalence classes of elements of $H^{m}(B)$ differing by constants; for $m=0$ it is denoted by $L^{2}(B) / \mathbb{R}$. We shall indicate by $L_{0}^{2}(B)$ the space of $L^{2}(B)$ functions having zero average over $B$. This notation will also be used for periodic Sobolev spaces without any other explicit reference to periodicity to avoid cumbersome notations.

2.1. Domain Partitioning and Finite Element Spaces. Let $\mathcal{T}_{h_{x}}^{x}$ and $\mathcal{T}_{h_{v}}^{v}$ be two families of cartesian partitions of $\Omega_{\mathbf{x}}$ and $\Omega_{\mathbf{v}}$, respectively, formed by rectangles for $d=2$ and cubes for $d=3$. Let $\left\{\mathcal{T}_{h}\right\}$ be defined as the cartesian product of these two partitions: $\mathcal{T}_{h}:=\mathcal{T}_{h_{x}}^{x} \times \mathcal{T}_{h_{v}}^{v}$; i.e.,

$$
\mathcal{T}_{h}:=\left\{\mathcal{R}=T^{x} \times T^{v}: \quad T^{x} \in \mathcal{T}_{h_{x}}^{x} \quad T^{v} \in \mathcal{T}_{h_{v}}^{v}\right\} .
$$

The mesh sizes $h, h_{x}$ and $h_{v}$ relative to the partitions are defined as usual

$$
0<h_{x}=\max _{T^{x} \in \mathcal{T}_{h_{x}}^{x}} \operatorname{diam}\left(T^{x}\right), \quad 0<h_{v}=\max _{T^{v} \in \mathcal{T}_{h_{v}}^{v}} \operatorname{diam}\left(T^{v}\right), \quad h=\max \left(h_{x}, h_{v}\right) .
$$

We denote by $\mathcal{E}_{\mathbf{x}}$ and $\mathcal{E}_{\mathbf{v}}$ the set of all edges of the partitions $\mathcal{T}_{h_{x}}^{x}$ and $\mathcal{T}_{h_{v}}^{v}$, respectively and we set $\mathcal{E}=\mathcal{E}_{\mathbf{x}} \times \mathcal{E}_{\mathbf{v}}$. The set of interior and boundary edges of the partition $\mathcal{T}_{h_{x}}^{x}$ (resp. $\left.\mathcal{T}_{h_{v}}^{v}\right)$ are denoted by $\mathcal{E}_{\mathbf{x}}^{0}\left(\operatorname{resp} . \mathcal{E}_{\mathbf{v}}^{0}\right)$ and $\mathcal{E}_{\mathbf{x}}^{\partial}\left(\right.$ resp. $\left.\mathcal{E}_{\mathbf{v}}^{\partial}\right)$, so that $\mathcal{E}_{\mathrm{x}}=\mathcal{E}_{\mathbf{x}}^{0} \cup \mathcal{E}_{\mathbf{x}}^{\partial}\left(\operatorname{resp} . \mathcal{E}_{\mathbf{v}}=\mathcal{E}_{\mathbf{v}}^{0} \cup \mathcal{E}_{\mathbf{v}}^{\partial}\right)$.

Trace operators: Observe that due to the structure of the transport equation (1), for each $\mathcal{R}=T^{x} \times T^{v} \in \mathcal{T}_{h}$ with $T^{x} \in \mathcal{T}_{h_{x}}^{x}$ and $T^{v} \in \mathcal{T}_{h_{v}}^{v}$ and for each $\varphi \in H^{1}\left(T^{x} \times T^{v}\right)$ we only need to define the traces of $\phi$ at $\partial T^{x} \times T^{v}$ and $T^{x} \times \partial T^{v}$. Hence, for setting the notation, it is enough to consider a general element $T$ in either $\mathcal{T}_{h_{x}}^{x}$ or $\mathcal{T}_{h_{v}}^{v}$. By $\mathbf{n}_{\mid \partial T}^{-}$we designate the outward normal to the element $T$ and we denote by $\varphi^{-}$the interior trace of $\varphi_{\left.\right|_{T}}$ on $\partial T$ and $\varphi^{+}$refers to the outer trace on $\partial T$ of $\varphi_{\left.\right|_{T}}$. That is,

$$
\varphi_{T}^{ \pm}(\mathbf{x}, \cdot)=\lim _{\epsilon \rightarrow 0} \varphi_{T}\left(\mathbf{x} \pm \epsilon \mathbf{n}^{-}, \cdot\right) \quad \forall \mathbf{x} \in \partial T .
$$

We next define the trace operators, but to avoid complications with fixing some privileged direction we follow [5]. Let $T_{-}$and $T_{+}$be two neighboring elements in either $\mathcal{T}_{h_{x}}^{x}$ or $\mathcal{T}_{h_{v}}^{v}$, and let $\mathbf{n}^{-}$and $\mathbf{n}^{+}$be their outward normal unit vectors, and let $\varphi^{ \pm}$and $\boldsymbol{\tau}^{ \pm}$be the restriction of $\varphi$ and $\boldsymbol{\tau}$ to $T_{ \pm}$. Following [5] we set:

$$
\{\varphi\}=\frac{1}{2}\left(\varphi^{-}+\varphi^{+}\right), \quad \llbracket \varphi \rrbracket=\varphi^{-} \mathbf{n}^{-}+\varphi^{+} \mathbf{n}^{+} \quad \text { on } e \in \mathcal{E}_{r}^{0}, \quad r=\mathbf{x} \text { or } \mathbf{v},
$$




$$
\{\boldsymbol{\tau}\}=\frac{1}{2}\left(\boldsymbol{\tau}^{-}+\boldsymbol{\tau}^{+}\right), \quad \llbracket \boldsymbol{\tau} \rrbracket=\boldsymbol{\tau}^{-} \cdot \mathbf{n}^{-}+\boldsymbol{\tau}^{+} \cdot \mathbf{n}^{+} \quad \text { on } e \in \mathcal{E}_{r}^{0}, \quad r=\mathbf{x} \text { or } \mathbf{v} .
$$

We also introduce a weighted average, for both scalar- and vector-valued functions, as follows. With each internal edge e, shared by elements $T^{+}$and $T^{-}$, we associate two real nonnegative numbers $\delta$ and $1-\delta$, and we define

$$
\{\boldsymbol{\tau}\}_{\delta}:=\delta \boldsymbol{\tau}^{+}+(1-\delta) \boldsymbol{\tau}^{-} \quad \text { on internal edges. }
$$

For $e \in \mathcal{E}_{r}^{\partial}$ (with $r=\mathbf{x}$ or $\mathbf{v}$ ), we set

$$
\llbracket \varphi \rrbracket=\varphi \mathbf{n}, \quad\{\varphi\}=\varphi, \quad\{\boldsymbol{\tau}\}=\boldsymbol{\tau} .
$$

Notice that when referring to elements rather than edges, according to $(5), \varphi^{-}$ can be seen as the inner trace relative to $T^{-}$(i.e., $\varphi_{T^{-}}^{-}$) and also as the outer trace relative to $T^{+}$(i.e., $\varphi_{T^{+}}^{+}$). Similarly, $\mathbf{n}^{-}$denotes the outward normal to $T^{-}$ and also the inner normal to $T^{+}$. Both notations will be used interchangeable.

Denoting by $\int_{\mathcal{E}_{r}}=\sum_{e \in \mathcal{E}_{r}} \int_{e}$, we shall make extensive use of the following identity (see [4])

$$
\sum_{T \in \mathcal{T}^{r}} \int_{\partial T^{r}} \boldsymbol{\tau} \cdot \mathbf{n} \varphi d s_{r}=\int_{\mathcal{E}_{r}}\{\boldsymbol{\tau}\} \cdot \llbracket \varphi \rrbracket d s_{r}+\int_{\mathcal{E}_{r}^{0}} \llbracket \boldsymbol{\tau} \rrbracket\{\varphi\} d s_{r} \quad r=\mathbf{x}, \mathbf{v} .
$$

Next, for $k \geq 0$, we define the discontinuous finite element spaces $V_{h}^{k}, \mathcal{Z}_{h}^{k}$ and $\Sigma_{h}^{k}$

$$
\begin{aligned}
& \mathcal{Z}_{h}^{k}:=\left\{\varphi \in L^{2}(\Omega): \quad \varphi_{\left.\right|_{\mathcal{R}}} \in \mathbb{Q}^{k}\left(T^{x}\right) \times \mathbb{Q}^{k}\left(T^{v}\right), \quad \forall \mathcal{R}=T^{x} \times T^{v} \in \mathcal{T}_{h}\right\}, \\
& X_{h}^{k}=\left\{\psi \in L^{2}\left(\Omega_{\mathbf{x}}\right): \quad \psi_{\left.\right|_{T^{x}}} \in \mathbb{Q}^{k}\left(T^{x}\right), \quad \forall T^{x} \in \mathcal{T}_{h_{x}}^{x}\right\}, \\
& V_{h}^{k}=\left\{\psi \in L^{2}\left(\Omega_{\mathbf{v}}\right): \quad \psi_{\left.\right|_{T^{v}}} \in \mathbb{Q}^{k}\left(T^{v}\right), \quad \forall T^{x} \in \mathcal{T}_{h_{x}}^{x}\right\}, \\
& \boldsymbol{\Xi}_{h}^{k}=\left\{\boldsymbol{\tau} \in\left(L^{2}\left(\Omega_{\mathbf{x}}\right)\right)^{d}: \quad \boldsymbol{\tau}_{\left.\right|_{T^{x}}} \in\left(\mathbb{Q}^{k}\left(T^{x}\right)\right)^{d} \quad \forall T^{x} \in \mathcal{T}_{h_{x}}^{x}\right\},
\end{aligned}
$$

where $\mathbb{Q}^{k}(T)$ (resp. $\left(\mathbb{Q}^{k}(T)^{d}\right)$ is the space of scalar (resp. vectorial) polynomials of degree at most $k$ in each variable.

We also set $Q_{h}^{k}=X_{h}^{k} \cap L_{0}^{2}\left(\Omega_{\mathbf{x}}\right)$. We finally introduce the Raviart-Thomas finite element space:

$$
\boldsymbol{\Sigma}_{h}^{k}=\left\{\boldsymbol{\tau} \in H\left(\operatorname{div} ; \Omega_{\mathbf{x}}\right): \quad \boldsymbol{\tau}_{\left.\right|_{T^{x}}} \in R T^{k}\left(T^{x}\right) \quad \forall T^{x} \in \mathcal{T}_{h_{x}}^{x}\right\}
$$

where

$H\left(\operatorname{div} ; \Omega_{\mathbf{x}}\right)=\left\{\boldsymbol{\tau} \in\left(L^{2}\left(\Omega_{\mathbf{x}}\right)\right)^{d}\right.$ with $\operatorname{div}(\boldsymbol{\tau}) \in L^{2}\left(\Omega_{\mathbf{x}}\right)$ and $\boldsymbol{\tau} \cdot \mathbf{n}_{\partial \Omega}$ periodic on $\left.\partial \Omega\right\}$ and $R T^{k}\left(T^{x}\right):=\mathbb{Q}^{k}\left(T^{x}\right)^{d}+\mathbf{x} \cdot \mathbb{Q}^{k}\left(T^{x}\right)$ (see [15] for further details). We shall denote by $\|\cdot\|_{H\left(\operatorname{div} ; \Omega_{\mathbf{x}}\right)}$ the $H\left(\operatorname{div} ; \Omega_{\mathbf{x}}\right)$-norm defined by

$$
\|\boldsymbol{\tau}\|_{H\left(\operatorname{div} ; \Omega_{\mathbf{x}}\right)}^{2}:=\|\boldsymbol{\tau}\|_{0}^{2}+\|\operatorname{div}(\boldsymbol{\tau})\|_{0}^{2} \quad \forall \boldsymbol{\tau} \in H\left(\operatorname{div} ; \Omega_{\mathbf{x}}\right) .
$$


2.2. Technical Tools. We start by defining the following seminorm and norms that will be used in our analysis:

$$
\begin{array}{lll}
|\varphi|_{1, h}^{2}=\sum_{\mathcal{R} \in \mathcal{T}_{h}}|\varphi|_{1, \mathcal{R}}^{2} & \|\varphi\|_{m, \mathcal{T}_{h}}^{2}:=\sum_{\mathcal{R} \in \mathcal{T}_{h}}\|\varphi\|_{m, \mathcal{R}}^{2} \quad \forall \varphi \in H^{m}\left(\mathcal{T}_{h}\right), m \geq 0 \\
\|\varphi\|_{0, \infty, \mathcal{T}_{h}}=\sup _{\mathcal{R} \in \mathcal{T}_{h}}\|\varphi\|_{0, \infty, \mathcal{R}} & \|\varphi\|_{L^{p}\left(\mathcal{T}_{h}\right)}^{p}:=\sum_{\mathcal{R} \in \mathcal{T}_{h}}\|\varphi\|_{L^{p}(\mathcal{R})}^{p} \quad \forall \varphi \in L^{p}\left(\mathcal{T}_{h}\right),
\end{array}
$$

for all $1 \leq p<\infty$. We also introduce the following norms over the skeleton of the finite element partition,

$$
\|\varphi\|_{0, \mathcal{E}_{x}}^{2}:=\sum_{e \in \mathcal{E}_{x}} \int_{e}|\varphi|^{2} d s_{x} d \mathbf{v}, \quad\|\varphi\|_{0, \mathcal{E}_{v}}^{2}=\sum_{e \in \mathcal{E}_{v}} \int_{e}|\varphi|^{2} d s_{v} d \mathbf{x} .
$$

Then, we define $\|\varphi\|_{0, \mathcal{E}_{h}}^{2}=\|\varphi\|_{0, \mathcal{E}_{x}}^{2}+\|\varphi\|_{0, \mathcal{E}_{v}}^{2}$.

Projection operators: Let $k \geq 0$ and let $\mathcal{P}_{h}: L^{2}(\Omega) \longrightarrow \mathcal{Z}_{h}^{k}$ be the standard $2 d-L^{2}$-projection. We denote by $\mathcal{P}_{\mathbf{x}}: L^{2}(\Omega) \longrightarrow X_{h}^{k}$ and $\mathcal{P}_{\mathbf{v}}: L^{2}(\Omega) \longrightarrow V_{h}^{k}$ the standard $d$-dimensional $L^{2}$-projections onto the spaces $X_{h}^{k}$ and $V_{h}^{k}$, respectively, and we note that $\mathcal{P}_{h}$ can be written as

$$
\mathcal{P}_{h}=\mathcal{P}_{\mathbf{x}} \otimes \mathcal{P}_{\mathbf{v}}
$$

The projection $\mathcal{P}_{h}$ satisfies (see [20] and [3])

(10) $\left\|w-\mathcal{P}_{h}(w)\right\|_{0, \mathcal{T}_{h}}+h^{1 / 2}\left\|w-\mathcal{P}_{h}(w)\right\|_{0, \mathcal{E}_{h}} \leq C h^{k+1}\|w\|_{k+1, \Omega} \quad \forall w \in H^{k+1}(\Omega)$,

with $C$ depending only on the shape regularity of the triangulation and the polynomial degree. By definition, $\mathcal{P}_{h}$ is stable in $L^{2}$ and it can be further shown to be stable in all $L^{p}$-norms (see [22] for details);

$$
\left\|\mathcal{P}_{h}(w)\right\|_{L^{p}\left(\mathcal{T}_{h}\right)} \leq C\|w\|_{L^{p}(\Omega)} \quad \forall w \in L^{p}(\Omega) \quad 1 \leq p \leq \infty .
$$

We will also need approximation properties in the supremum-norm (see [20]);

$$
\left\|w-\mathcal{P}_{h}(w)\right\|_{0, \infty, \mathcal{T}_{h}} \leq C h^{k+1}\|w\|_{k+1, \infty, \Omega} \quad \forall w \in W^{k+1, \infty}(\Omega) .
$$

We wish to stress that the projections $\mathcal{P}_{\mathbf{x}}$ and $\mathcal{P}_{\mathbf{v}}$ also satisfy properties (11) and (12). Furthermore, we will also use

$$
\left\|w-\mathcal{P}_{\mathbf{r}}(w)\right\|_{0, \mathcal{T}_{h}} \leq C h^{k+1}\|w\|_{k+1, \Omega} \quad \forall w \in H^{k+1}(\Omega), \quad \mathbf{r}=\mathbf{x} \text { or } \mathbf{v} .
$$

Raviart Thomas projection: For $k \geq 0$ we denote by $\mathcal{R}_{h}^{k}$ the local interpolation operator which satisfies the following commuting diagram:

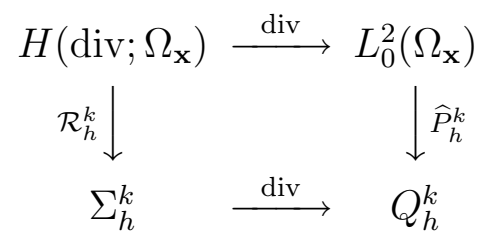


where $\widehat{P}_{h}^{k}$ refers to the standard $L^{2}$-projection operator onto $Q_{h}^{k}$. The above commuting diagram express that $\operatorname{div}\left(\sum_{h}^{k}\right)=Q_{h}^{k}$ and

$$
\operatorname{div} \mathcal{R}_{h}^{k}(\boldsymbol{\tau})=\widehat{P}_{h}^{k}(\operatorname{div} \boldsymbol{\tau}) \quad \forall \boldsymbol{\tau} \in H\left(\operatorname{div} ; \Omega_{\mathbf{x}}\right) .
$$

In particular (14) holds for all $\boldsymbol{\tau} \in H^{1}\left(\Omega_{\mathbf{x}}\right)^{d}$. Optimal $L^{p}$-approximation properties, with $2 \leq p \leq \infty$ can be shown for this operator (see [15, Section III.3], $[15,27]$ for details):

$$
\begin{aligned}
\left\|\boldsymbol{\tau}-\mathcal{R}_{h}^{k}(\boldsymbol{\tau})\right\|_{L^{p}\left(\Omega_{\mathbf{x}}\right)}+\left\|\operatorname{div}\left(\boldsymbol{\tau}-\mathcal{R}_{h}^{k}(\boldsymbol{\tau})\right)\right\|_{L^{p}\left(\Omega_{\mathbf{x}}\right)} \leq C h^{k+1}\|\boldsymbol{\tau}\|_{W^{k+1, p}\left(\Omega_{\mathbf{x}}\right)} \\
\forall \boldsymbol{\tau} \in W^{k+1, p}\left(\Omega_{\mathbf{x}}\right)^{d} .
\end{aligned}
$$

We notice here that all the approximation and stability results stated here for the standard $L^{2}$-projection, hold true also for the $L^{2}$-projection onto $Q_{h}^{k}$; i.e., $\widehat{P}_{h}^{k}$.

\section{Numerical Methods and Stability}

In this section we describe the numerical methods we propose for approximating the Vlasov-Poisson system (1)-(3) and prove Stability for the proposed schemes. Following the work initiated in [6], the proposed numerical schemes are based on the coupling of a simple DG discretization of the Vlasov equation and some suitable finite element approximation, possibly discontinuous, to the Poisson problem.

Thanks to the special hamiltonian structure of the Vlasov equation (1): $\mathbf{v}$ is independent of $\mathbf{x}$ and $\mathbf{E}$ is independent of $\mathbf{v}$; for all methods the DG approximation for the electron distribution function is done exactly in the same way. Therefore we first present the DG method for the transport equation (1), postponing the description of the approximation to the Poisson problem (3) to the last part of the section.

While describing the numerical schemes, we will also state a number of approximation results. The proofs of most of them, except for the stability and particle conservation, are postponed till Appendix A.

\subsection{Discontinuous Galerkin approximation for the Vlasov equation.}

Throughout this section, we denote by $\mathbf{E}_{h} \in \widetilde{\Sigma}$ the FE approximation to the electrostatic field to be specified later on. We consider DG approximation for the Vlasov equation coupled with a finite element approximation to the Poisson problem. The DG approximation to (1) reads: Find $\left(\mathbf{E}_{h}, f_{h}\right) \in \mathcal{C}^{1}\left([0, T] ; \widetilde{\Sigma} \times \mathcal{Z}_{h}^{k}\right)$ such that

$$
\sum_{R \in \mathcal{T}_{h}} \mathcal{B}_{h, R}\left(\mathbf{E}_{h} ; f_{h}, \varphi_{h}\right)=0 \quad \forall \phi_{h} \in \mathcal{Z}_{h}^{k}
$$

where $\forall R=T^{x} \times T^{v} \in \mathcal{T}_{h}$,

$$
\mathcal{B}_{h, R}\left(\mathbf{E}_{h} ; f_{h}, \varphi_{h}\right)=\int_{R} \frac{\partial f_{h}}{\partial t} \varphi_{h} d \mathbf{v} d \mathbf{x}-\int_{R} f_{h} \mathbf{v} \cdot \nabla_{x} \varphi_{h} d \mathbf{v} d \mathbf{x}+\int_{R} f_{h} \mathbf{E}_{h} \cdot \nabla_{\mathbf{v}} \varphi_{h} d \mathbf{v} d \mathbf{x}
$$




$$
+\int_{T^{v}} \int_{\partial T^{x}}\left(\widehat{\mathbf{v} \cdot \mathbf{n} f_{h}}\right) \varphi_{h} d s_{x} d \mathbf{v}-\int_{T^{x}} \int_{\partial T^{v}}\left(\widehat{\mathbf{E}_{h} \cdot \mathbf{n} f_{h}}\right) \varphi_{h} d s_{v} d \mathbf{x} \quad \forall \varphi_{h} \in \mathcal{Z}_{h}^{k} .
$$

Above, we have used $\mathbf{n}$ to denote both $\mathbf{n}_{\left.\right|_{\partial T^{x}} ^{-}}^{-}$and $\mathbf{n}_{\left.\right|_{\partial T^{v}}}^{-}$in the first and second boundary integrals respectively. To ease the presentation, this slight abuse in the notation will be used throughout the paper, since it will be usually clear to which normal we are referring. The numerical fluxes $\left(\widehat{\mathbf{v} \cdot \mathbf{n}^{-}} f_{h}\right)$ and $\left(\widehat{\mathbf{E}_{h} \cdot \mathbf{n}^{-}} f_{h}\right)$ are defined as:

$$
\begin{gathered}
\widehat{\mathbf{v} \cdot \mathbf{n}^{-} f_{\left.h\right|_{\partial T^{x}}}}= \begin{cases}\mathbf{v} \cdot \mathbf{n}^{-}\left(f_{h}\right)_{\left.\right|_{T^{x}}}^{-} & \text {if } \mathbf{v} \cdot \mathbf{n}_{\left.\right|_{\partial T^{x}} ^{-}}^{-}>0, \\
\mathbf{v} \cdot \mathbf{n}^{-}\left(f_{h}\right)_{\left.\right|_{T^{x}}}^{+} & \text {if } \mathbf{v} \cdot \mathbf{n}_{\left.\right|_{\partial T^{x}}}^{-}<0, \\
\left\{\mathbf{v} \cdot \mathbf{n}^{-} f_{h}\right\} & \text { if } \mathbf{v} \cdot \mathbf{n}_{\left.\right|_{\partial T^{x}}}^{-}=0,\end{cases} \\
\widehat{\mathbf{E}_{h} \cdot \mathbf{n}^{-}} f_{\left.h\right|_{\partial T^{v}}}= \begin{cases}\mathbf{E}_{h} \cdot \mathbf{n}^{-}\left(f_{h}\right)_{\left.\right|_{T^{v}}}^{+} & \text {if } \mathbf{E}_{h} \cdot \mathbf{n}_{\left.\right|_{\partial T^{v}}}^{-}>0, \\
\mathbf{E}_{h} \cdot \mathbf{n}^{-}\left(f_{h}\right)_{\left.\right|_{T^{v}}}^{-} & \text {if } \mathbf{E}_{h} \cdot \mathbf{n}_{\left.\right|_{\partial T^{v}}}^{-}<0, \\
\left\{\mathbf{E}_{h} \cdot \mathbf{n}^{-} f_{h}\right\} & \text { if } \mathbf{E}_{h} \cdot \mathbf{n}_{\left.\right|_{\partial T^{v}}}^{-}=0,\end{cases}
\end{gathered}
$$

on interior edges, i.e., for all $\partial T^{x} \cap \partial \Omega_{\mathbf{x}}=\emptyset$ and $\partial T^{v} \cap \partial \Omega_{\mathbf{v}}=\emptyset$. On boundary edges we impose the periodicity for $\widehat{\mathbf{v} \cdot \mathbf{n} f}$ and compactness for $\widehat{\mathbf{E}_{h} \cdot \mathbf{n} f}$. Notice that the (upwind) fluxes defined in (17) are consistent and conservative. Now, taking into account the definition of the weighted average (8) and that of the standard trace operators (6) and (7) and the fact that for each fixed $e, \mathbf{n}^{-}=-\mathbf{n}^{+}$, the upwind numerical fluxes (17) can be re-written in terms of the weighted average (see $[16,8]$ for details). More precisely, we have

$$
\begin{cases}\widehat{\mathbf{v} \cdot \mathbf{n} f_{h}}=\left\{\mathbf{v} f_{h}\right\}_{\alpha} \cdot \mathbf{n}:=\left(\left\{\mathbf{v} f_{h}\right\}+\frac{|\mathbf{v} \cdot \mathbf{n}|}{2} \llbracket f_{h} \rrbracket\right) \cdot \mathbf{n} & \text { on } \mathcal{E}_{x}^{0}, \\ \widehat{\mathbf{E}_{h} \cdot \mathbf{n} f_{h}}=\left\{\mathbf{E}_{h} f_{h}\right\}_{\beta} \cdot \mathbf{n}:=\left(\left\{\mathbf{E}_{h} f_{h}\right\}-\frac{\left|\mathbf{E}_{h} \cdot \mathbf{n}\right|}{2} \llbracket f_{h} \rrbracket\right) \mathbf{n} & \text { on } \mathcal{E}_{v}^{0},\end{cases}
$$

with $\alpha=\frac{1}{2}\left(1 \pm \operatorname{sign}\left(\mathbf{v} \cdot \mathbf{n}^{ \pm}\right)\right)$and $\beta=\frac{1}{2}\left(1 \mp \operatorname{sign}\left(\mathbf{E}_{h} \cdot \mathbf{n}^{ \pm}\right)\right)$. Using then, formula (9) together with the conservativity property of the numerical fluxes, the DG scheme reads

$$
\begin{aligned}
0= & \sum_{\mathcal{R} \in \mathcal{T}_{h}} \mathcal{B}_{h, R}\left(\mathbf{E}_{h} ; f_{h}, \varphi_{h}\right) \\
= & \sum_{\mathcal{R} \in \mathcal{T}_{h}} \int_{\mathcal{R} \mathcal{R}} \frac{\partial f_{h}}{\partial t} \varphi_{h} d \mathbf{v} d \mathbf{x}-\int_{\Omega} f_{h} \mathbf{v} \cdot \nabla_{\mathbf{x}}^{h} \varphi_{h} d \mathbf{v} d \mathbf{x}+\int_{\Omega} f_{h} \mathbf{E}_{h} \cdot \nabla_{\mathbf{v}}^{h} \varphi_{h} d \mathbf{v} d \mathbf{x} \\
& +\int_{T^{v}} \int_{\mathcal{E}_{x}}\left\{\mathbf{v} f_{h}\right\}_{\alpha} \cdot \llbracket \varphi_{h} \rrbracket d s_{x} d \mathbf{v}-\int_{T^{x}} \int_{\mathcal{E}_{v}}\left\{\mathbf{E}_{h} f_{h}\right\}_{\beta} \cdot \llbracket \varphi_{h} \rrbracket d s_{v} d \mathbf{x} \quad \forall \varphi_{h} \in \mathcal{Z}_{h}^{k},
\end{aligned}
$$

where $\nabla_{\mathbf{x}}^{h} \varphi_{h}$ and $\nabla_{\mathbf{v}}^{h} \varphi_{h}$ are the functions whose restriction to each element $\mathcal{R} \in \mathcal{T}_{h}$ are equal to $\nabla_{\mathbf{x}} \varphi_{h}$ and $\nabla_{\mathbf{v}} \varphi_{h}$, respectively. 
The discrete density, $\rho_{h}$ is defined by

$$
\rho_{h}=\sum_{T^{v} \in \mathcal{T}_{h_{v}^{v}}^{v}} \int_{T^{v}} f_{h} d \mathbf{v} \in X_{h}^{k} .
$$

The following lemma guarantees the particle conservation for the above scheme.

Lemma 1 (Particle or Mass Conservation). Let $k \geq 0$ and let $f_{h} \in \mathcal{C}^{1}\left([0, T] ; \mathcal{Z}_{h}^{k}\right)$ be the DG aproximation to $f$, satisfying (16). Then,

$$
\sum_{\mathcal{R} \in \mathcal{T}_{h}} \int_{\mathcal{R}} f_{h}(t) d \mathbf{x} d \mathbf{v}=\sum_{\mathcal{R} \in \mathcal{T}_{h}} \int_{\mathcal{R}} f_{h}(0) d \mathbf{x} d \mathbf{v}=\sum_{\mathcal{R} \in \mathcal{T}_{h}} \int_{\mathcal{R}} f_{0}=1 \quad \forall t .
$$

Proof. The proof follows essentially the same lines as the proof of [6, Lemma 3.1], by fixing some arbitrary $\mathcal{R}=\mathcal{R}_{1}$ and taking in (16) a test function $\varphi$, such that $\varphi_{h}=1$ in $\mathcal{R}_{1}$ and $\varphi_{h}=0$ elsewhere.

We next show $L^{2}$-stability for the numerical method (16), which follows from the selection of the numerical fluxes:

Proposition 1 ( $L^{2}$-stability). Let $f_{h} \in \mathcal{Z}_{h}^{k}$ be the approximation of problem (1)(3), solution of (16) with the numerical fluxes defined as in (17). Then

$$
\left\|f_{h}(t)\right\|_{0, \mathcal{T}_{h}} \leq\left\|f_{h}(0)\right\|_{0, \mathcal{T}_{h}} \quad \forall t \in[0, T] .
$$

Proof. The proof follows essentially the same steps as for the case $d=1$. By setting $\varphi_{h}=f_{h}$ in (16), integrating the volume terms that result and using (9) one easily gets

$$
\begin{aligned}
0= & \sum_{\mathcal{R} \in \mathcal{T}_{h}} \mathcal{B}_{h, R}\left(\mathbf{E}_{h} ; f_{h}, f_{h}\right) \\
= & \frac{1}{2} \sum_{\mathcal{R} \in \mathcal{T}_{h}}\left(\frac{d}{d t} \int_{\mathcal{R}} f_{h}^{2} d \mathbf{v} d \mathbf{x}-\int_{T^{v}} \int_{\mathcal{E}_{x}} \mathbf{v} \cdot \llbracket f_{h}^{2} \rrbracket d s_{x} d \mathbf{v}+\int_{T^{x}} \int_{\mathcal{E}_{v}} \mathbf{E}_{h} \cdot \llbracket f_{h}^{2} \rrbracket d s_{v} d \mathbf{x}\right) \\
& +\sum_{T^{v} \in \mathcal{T}_{h_{v}}^{v}} \int_{T^{v}} \int_{\mathcal{E}_{x}}\left\{\mathbf{v} f_{h}\right\}_{\alpha} \cdot \llbracket f_{h} \rrbracket d s_{x} d \mathbf{v}-\sum_{T^{x} \in \mathcal{T}_{h_{x}}^{x}} \int_{T^{x}} \int_{\mathcal{E}_{v}}\left\{\mathbf{E}_{h} f_{h}\right\}_{\beta} \cdot \llbracket f_{h} \rrbracket d s_{v} d \mathbf{x} .
\end{aligned}
$$

Now, from the definition of the trace operators (6) it follows that $\llbracket f_{h}^{2} \rrbracket=2\left\{f_{h}\right\} \llbracket f_{h} \rrbracket$ on $e \in \mathcal{E}_{h}^{0}$. Substituting the above identity together with the definition of the numerical fluxes given in (18), and using the periodic boundary conditions in $\mathbf{x}$ and compact support in $\mathbf{v}$, we have that

$$
\begin{aligned}
0= & \frac{1}{2} \frac{d}{d t} \int_{\Omega} f_{h}^{2} d \mathbf{v} d \mathbf{x} \\
& +\sum_{T^{v} \in \mathcal{T}_{h_{v}}^{v}} \int_{T^{v}} \int_{\mathcal{E}_{x}^{0}} \frac{|\mathbf{v} \cdot \mathbf{n}|}{2} \llbracket f_{h} \rrbracket^{2} d s_{x} d \mathbf{v}+\sum_{T^{x} \in \mathcal{T}_{h_{x}}^{x}} \int_{T^{x}} \int_{\mathcal{E}_{v}^{0}} \frac{\left|\mathbf{E}_{h} \cdot \mathbf{n}\right|}{2} \llbracket f_{h} \rrbracket^{2} d s_{v} d \mathbf{x} .
\end{aligned}
$$

Integration in time of the above equation, from 0 to $t$ concludes the proof. 
We close this section stating an elementary approximation result that will be required in our analysis. Its proof is given in Appendix A.

Lemma 2. Let $k \geq 0$ and $f$ and $f_{h}$ be the continuous and approximate solutions to the Vlasov-Poisson problem. Let $\rho$ and $\rho_{h}$ be the continuous and discrete densities defined in (2) and (23). Then,

$$
\left\|\rho-\rho_{h}\right\|_{0, \mathcal{T}_{h x}^{x}} \leq C\left[\operatorname{meas}\left(\Omega_{\mathbf{v}}\right)\right]^{1 / 2}\left\|f-f_{h}\right\|_{0, \mathcal{T}_{h}} \leq C L^{d / 2}\left\|f-f_{h}\right\|_{0, \mathcal{T}_{h}} .
$$

Furthermore, if $\rho \in W^{3 / 2, d}\left(\Omega_{\mathbf{x}}\right)$ and $f \in \mathcal{C}^{1}\left([0, T] ; H^{k+1}(\Omega)\right)$ we have,

$$
\begin{aligned}
\left\|\rho-\rho_{h}\right\|_{-1, \infty, \mathcal{T}_{h_{x}}^{x}} \leq C h^{3 / 2}\|\rho\|_{W^{3 / 2, d}\left(\Omega_{\mathrm{x}}\right)} & \\
& \quad+C L^{d / 2} h^{1-d / 2}\left(C h^{k+1}\|f\|_{k+1, \Omega}+\left\|f_{h}-\mathcal{P}_{h}(f)\right\|_{0, \mathcal{T}_{h}}\right) .
\end{aligned}
$$

3.2. Mixed Finite Element Approximation to the Poisson problem. We next consider the approximation to the discrete Poisson problem, which can be rewritten as the following first order system:

$$
\mathbf{E}=\nabla_{\mathbf{x}} \Phi \quad \text { in } \Omega_{\mathbf{x}}, \quad-\operatorname{div}_{\mathbf{x}}(\mathbf{E})=\rho_{h}-1 \quad \text { in } \Omega_{\mathbf{x}}, \quad \rho_{h}=\sum_{T^{v} \in \mathcal{T}_{h_{v}}^{v}} \int_{T^{v}} f_{h} d \mathbf{v}
$$

with periodic boundary conditions for $\mathbf{E}$ and $\Phi$. Notice that in view of Lemma 1 and by taking $\Phi \in L_{0}^{2}\left(\Omega_{\mathbf{x}}\right)$, we guarantee that the above problem is well posed. The weak formulation of the above problem reads: Find $(\mathbf{E}, \Phi) \in H\left(\operatorname{div} ; \Omega_{\mathbf{x}}\right) \times L_{0}^{2}(\Omega)$ such that

$$
\begin{array}{cl}
\int_{\Omega_{\mathbf{x}}} \mathbf{E} \cdot \boldsymbol{\tau} d \mathbf{x}=\int_{\Omega_{\mathbf{x}}} \nabla_{x} \Phi \cdot \boldsymbol{\tau} d \mathbf{x}=0 & \forall \boldsymbol{\tau} \in H\left(\operatorname{div} ; \Omega_{\mathbf{x}}\right), \\
-\int_{\Omega_{\mathbf{x}}} \operatorname{div}_{\mathbf{x}}(\mathbf{E}) q d \mathbf{x}=\int_{\Omega_{\mathbf{x}}}\left(\rho_{h}-1\right) q d \mathbf{x} & \forall q \in L_{0}^{2}(\Omega) .
\end{array}
$$

Unlike for the $1 D$ case, where direct integration of the Poisson equation provides a conforming finite element approximation to the electrostatic potential (see [6]), for higher dimensions we only consider mixed finite element approximation to the discrete Poisson problem with either Raviart-Thomas or DG elements. Throughout this section, we focus on the detailed description of the methods we consider, stating also the approximation results that will be needed in our subsequent error analysis. However, the proofs of all these results are postponed to $\S \mathrm{A}$. We next describe each of these approaches in detail.

3.2.1. Raviart-Thomas mixed finite element approximation. The approximation reads: find $\left(\mathbf{E}_{h}, \Phi_{h}\right) \in \Sigma_{h}^{r} \times Q_{h}^{r}$ satisfying

$$
\begin{array}{rlrl}
\int_{\Omega_{\mathbf{x}}} \mathbf{E}_{h} \cdot \boldsymbol{\tau} d \mathbf{x} & +\int_{\Omega_{\mathbf{x}}} \Phi_{h} \operatorname{div}_{\mathbf{x}}(\boldsymbol{\tau}) d \mathbf{x}=0 & \forall \boldsymbol{\tau} \in \boldsymbol{\Sigma}_{h}^{k}, \\
-\int_{\Omega_{\mathbf{x}}} \operatorname{div}_{\mathbf{x}}\left(\mathbf{E}_{h}\right) q d \mathbf{x}=\int_{\Omega_{\mathbf{x}}}\left(\rho_{h}-1\right) q d \mathbf{x} & \forall q \in Q_{h}^{k} .
\end{array}
$$


The following lemma provides error estimates in the above norm for the approximate electrostatic field. Its proof is given in Appendix A.

Lemma 3. Let $k \geq 0$ and let $\left(\mathbf{E}_{h}, \Phi_{h}\right) \in \mathcal{C}^{0}\left([0, T] ; \Sigma_{h}^{k} \times X_{h}^{k}\right)$ be the $R T_{k}$ approximation to the Poisson problem (23). Assume $\Phi \in \mathcal{C}^{0}\left([0, T] ; H^{k+2}\left(\Omega_{\mathbf{x}}\right)\right)$. Then, the following estimates hold for all $t \in[0, T]$ :

$$
\left\|\mathbf{E}(t)-\mathbf{E}_{h}(t)\right\|_{H\left(\operatorname{div} ; \Omega_{\mathbf{x}}\right)} \leq C h^{k+1}\|\Phi(t)\|_{k+2, \Omega_{\mathbf{x}}}+C L^{d / 2}\left\|f(t)-f_{h}(t)\right\|_{0, \mathcal{T}_{h}} .
$$

For our error analysis, we also need an estimate for the $L^{\infty}$-error of the electrostatic field. This is given in next result, whose proof can be found in Appendix A.

Lemma 4. Let $k \geq 0$ and let $\left(\mathbf{E}_{h}, \Phi_{h}\right) \in \mathcal{C}^{0}\left([0, T] ; \Sigma_{h}^{k} \times X_{h}^{k}\right)$ be the $R T_{k}$ approximation to the Poisson problem (23). Then, the following estimate hold for all $t \in[0, T]:$

$$
\left\|\mathbf{E}(t)-\mathbf{E}_{h}(t)\right\|_{0, \infty, \Omega_{\mathbf{x}}} \leq C\left\|\mathbf{E}-\mathcal{R}_{h}^{k}(\mathbf{E})\right\|_{1, \infty, \Omega_{\mathbf{x}}}+C|\log (h)|\left\|\rho-\rho_{h}\right\|_{-1, \infty, \Omega_{\mathbf{x}}} .
$$

Remark 1. We could have considered also Brezzi-Douglas-Marini BDM [13, 14] or Brezzi-Douglas-Fortin-Marini BDFM [12] finite elements for the approximation of the Poisson problem. We wish to stress that all the results shown in this paper for the Raviart-Thomas -DG method for Vlasov-Poisson remain valid if the $\mathrm{RT}_{k}$ approximation for the Poisson problem is replaced by a $\mathrm{BDM}_{k+1}$ or $\mathrm{BDFM}_{k+1}$ approximation. See also [15] for further details on the approximation with these elements.

3.2.2. Discontinuous Galerkin approximation. For $r \geq 1$ the method reads: find $\left(\mathbf{E}_{h}, \Phi_{h}\right) \in \boldsymbol{\Xi}_{h}^{r} \times Q_{h}^{r}$ such that

$$
\begin{array}{cl}
\int_{T^{x}} \mathbf{E}_{h} \cdot \boldsymbol{\tau} d \mathbf{x}+\int_{T^{x}} \Phi_{h} \operatorname{div}_{\mathbf{x}}(\boldsymbol{\tau}) d \mathbf{x}-\int_{\partial T^{x}} \widehat{\Phi_{h}} \boldsymbol{\tau} \cdot \mathbf{n} d s_{x}=0 & \forall \boldsymbol{\tau} \in \mathbf{\Xi}_{h}^{r} \\
\int_{T^{x}} \mathbf{E}_{h} \cdot \nabla_{\mathbf{x}} q d \mathbf{x}-\int_{\partial T^{x}} q \widehat{\mathbf{E}_{h}} \cdot \mathbf{n} d s_{x}=\int_{T^{x}}\left(\rho_{h}-1\right) q d \mathbf{x} & \forall q \in Q_{h}^{r} .
\end{array}
$$

On interior edges, the numerical fluxes are defined as

$$
\left\{\begin{array}{l}
\widehat{\mathbf{E}_{h}}=\left\{\mathbf{E}_{h}\right\}-\mathbf{C}_{12} \llbracket \mathbf{E}_{h} \rrbracket-C_{11} \llbracket \Phi_{h} \rrbracket, \\
\widehat{\Phi_{h}}=\left\{\Phi_{h}\right\}+\mathbf{C}_{12} \cdot \llbracket \Phi_{h} \rrbracket-C_{22} \llbracket \mathbf{E}_{h} \rrbracket,
\end{array}\right.
$$

and on boundary edges we impose the periodicity for both $\widehat{\mathbf{E}_{h}}$ and $\widehat{\Phi_{h}}$. As for the case $d=1$, the parameters $C_{11}, \mathbf{C}_{12}$ and $C_{22}$ could be taken in several ways to try to achieve different levels of accuracy. However, all superconvergence results for the Hybridized DG (in $d \geq 2$ ) are for partitions made of simplices (and the proof of these results rely strongly on that). As for the minimal dissipation MD-DG method (see [21] for details) one can expect at most, an improvement of half an order in the error estimate for $\left\|\mathbf{E}-\mathbf{E}_{h}\right\|_{0, \mathcal{T}_{h_{x}}}$ for $d=2$ (for a Poisson problem with dirichlet boundary conditions). Therefore, throughout this section we will not further distinguish between the possible choices (since no improvement on the final rate of convergence could be achieved) and we set $r=k+1$. One might 
stick to the classical LDG method for which $C_{22}=0$ and $C_{11}=c h^{-1}$ with $c$ a strictly positive constant. See [5].

Substituting the definition of the numerical fluxes (29) into (27)-(28) and summing over all elements of $\mathcal{T}_{h_{x}}^{x}$ we arrive at the mixed problem:

$$
\begin{cases}a\left(\mathbf{E}_{h}, \boldsymbol{\tau}\right)+b\left(\boldsymbol{\tau}, \Phi_{h}\right)=0 & \forall \boldsymbol{\tau} \in \mathbf{\Xi}_{h}^{r}, \\ -b\left(\mathbf{E}_{h}, q\right)+c\left(\Phi_{h}, q\right)=\int_{\Omega_{\mathbf{x}}}\left(\rho_{h}-1\right) q d \mathbf{x} & \forall q \in Q_{h}^{r},\end{cases}
$$

where

$$
\begin{aligned}
a\left(\mathbf{E}_{h}, \boldsymbol{\tau}\right) & =\int_{\Omega_{\mathbf{x}}} \mathbf{E}_{h} \cdot \boldsymbol{\tau} d \mathbf{x}, \\
b\left(\boldsymbol{\tau}, \Phi_{h}\right) & =\int_{\Omega_{\mathbf{x}}} \Phi_{h} \nabla_{\mathbf{x}}^{h} \cdot \boldsymbol{\tau} d \mathbf{x}-\int_{\mathcal{E}_{x}^{0}}\left(\left\{\Phi_{h}\right\}+\mathbf{C}_{12} \cdot \llbracket \Phi_{h} \rrbracket\right) \llbracket \boldsymbol{\tau} \rrbracket d s_{x}-\int_{\mathcal{E}_{x}^{\partial}} \Phi_{h} \boldsymbol{\tau} \cdot \mathbf{n} d s_{x}, \\
c\left(\Phi_{h}, q\right) & =\int_{\mathcal{E}_{x}} C_{11} \llbracket \Phi_{h} \rrbracket \cdot \llbracket q \rrbracket d s_{x} .
\end{aligned}
$$

Note that integration by parts of the volume term in $b\left(\boldsymbol{\tau}, \Phi_{h}\right)$ together with (9) gives

$$
b\left(\boldsymbol{\tau}, \Phi_{h}\right)=-\int_{\Omega_{\mathbf{x}}} \nabla_{\mathbf{x}}^{h} \Phi_{h} \cdot \boldsymbol{\tau} d \mathbf{x}+\int_{\mathcal{E}_{x}^{0}} \llbracket \Phi_{h} \rrbracket \cdot\left(\{\boldsymbol{\tau}\}-\mathbf{C}_{12} \llbracket \boldsymbol{\tau} \rrbracket\right) d s_{x}+\int_{\mathcal{E}_{x}^{\partial}} \Phi_{h} \boldsymbol{\tau} \cdot \mathbf{n} d s_{x} .
$$

We define the semi-norm

$$
|(\boldsymbol{\tau}, q)|_{\mathcal{A}}^{2}:=\|\boldsymbol{\tau}\|_{0, \mathcal{I}_{h}}^{2}+\left\|C_{11}^{1 / 2} \llbracket q \rrbracket\right\|_{0, \mathcal{E}_{x}}^{2}
$$

Next result provides the error estimates in the above norm for the approximation $\left(\mathbf{E}_{h}, \Phi_{h}\right)$ :

Lemma 5. Let $r \geq 1$ and let $\left(\mathbf{E}_{h}, \Phi_{h}\right) \in \mathcal{C}^{0}\left([0, T] ; \mathbf{\Xi}_{h}^{r} \times Q_{h}^{r}\right)$ be the LDG approximation to the Poisson problem solution of $(27)-(28)$. Assume $(\mathbf{E}, \Phi) \in$ $\mathcal{C}^{0}\left([0, T] ; H^{r+1}\left(\Omega_{\mathbf{x}}\right) \times H^{r+2}\left(\Omega_{\mathbf{x}}\right)\right)$. Then, the following estimates hold for all $t \in$ $[0, T]$ :

$$
\left|\left(\mathbf{E}(t)-\mathbf{E}_{h}(t), \Phi(t)-\Phi_{h}(t)\right)\right|_{\mathcal{A}} \leq C h^{s}\|\Phi(t)\|_{r+2, \Omega_{\mathbf{x}}}+C L^{d / 2}\left\|f(t)-f_{h}(t)\right\|_{0, \mathcal{T}_{h}} .
$$

Finally we state a result that gives the $L^{\infty}$-error estimate for the LDG approximation to (3) that will be required by our analysis. The proof is given in Appendix A.

Lemma 6. Let $r \geq 1$ and let $\left(\mathbf{E}_{h}, \Phi_{h}\right) \in \mathcal{C}^{0}\left([0, T] ; \mathbf{\Xi}_{h}^{r} \times Q_{h}^{r}\right)$ be the LDG approximation to the Poisson problem solution of (27)-(28). Then, the following estimate hold for all $t \in[0, T]$ :

$$
\begin{array}{r}
\left\|\mathbf{E}-\mathbf{E}_{h}\right\|_{0, \infty} \leq C|\log (h)|^{\bar{r}}\left(\left\|\mathbf{E}-\mathcal{P}^{x}(\mathbf{E})\right\|_{0, \infty, \Omega_{\mathbf{x}}}+h^{-1}\left\|\Phi-\mathcal{P}^{x}(\Phi)\right\|_{0, \infty, \Omega_{\mathbf{x}}}\right) \\
+C|\log (h)|\left\|\rho-\rho_{h}\right\|_{-1, \infty, \mathcal{T}_{h_{x}}^{x}}
\end{array}
$$

where $\bar{r}=1$ if $r=1$ and $\bar{r}=0$ for $r>1$. 


\section{Main Results and Error Analysis}

In this section, we now carry out the error analysis for the proposed DG approximations for the Vlasov-Poisson system. We first state and discuss our main results, and, then, we display the main ideas of our error analysis.

4.1. Main Results. The main result of this section is the following theorem:

Theorem 1. Let $\Omega=\Omega_{\mathbf{x}} \times \Omega_{\mathbf{v}}=[0,1]^{d} \times[-L, L]^{d} \subset \mathbb{R}^{2 d}, d=2,3$. Let $k \geq 1$ and let $f \in \mathcal{C}^{1}\left([0, T] ; H^{k+2}(\Omega) \cap W^{1, \infty}(\Omega)\right)$ be the compactly supported solution at time $t \in[0, T]$ of the Vlasov-Poisson problem (1)-(3) and let $\mathbf{E} \in$ $\mathcal{C}^{0}\left([0, T] ; H^{k+1}\left(\Omega_{\mathbf{x}}\right)^{d} \cap W^{1, \infty}\left(\Omega_{\mathbf{x}}\right)^{d}\right)$ with $d=2$ or 3 be the associated electrostatic potential. Then,

(a). $\mathbf{R T}_{k}$-DG method: if $\left(\left(\mathbf{E}_{h}, \Phi_{h}\right), f_{h}\right) \in \mathcal{C}^{0}\left([0, T] ;\left(\Sigma_{h}^{k} \times Q_{h}^{k}\right)\right) \times \mathcal{C}^{1}([0, T]$; $\left.\mathcal{Z}_{h}^{k}\right)$ is the $R T_{k}-D G$ approximation solution of $(19)-(24)$, the following estimates hold

$$
\left\|f(t)-f_{h}(t)\right\|_{0, \Omega} \leq C_{a} h^{k+1} \quad \forall t \in[0, T],
$$

where $C_{a}$ depends on the final time $T$, the polynomial degree $k$, the shape regularity of the partition and depends also on $f$ through the norms

$C_{a}=C_{a}\left(\|f(t)\|_{k+2, \Omega},\left\|f_{t}(t)\right\|_{k+1, \Omega},\|f\|_{1, \infty, \Omega},\|\Phi\|_{k+2, \Omega_{\mathbf{x}}},\|\mathbf{E}\|_{1, \infty, \Omega_{\mathbf{x}}}\right)$.

(b). DG-DG method: let $r=k+1$ and let $\left(\left(\mathbf{E}_{h}, \Phi_{h}\right), f_{h}\right) \in \mathcal{C}^{0}([0, T]$; $\left.\boldsymbol{\Xi}_{h}^{r} \times Q_{h}^{r}\right) \times \mathcal{C}^{1}\left([0, T] ; \mathcal{Z}_{h}^{k}\right)$ be the $D G-D G$ approximation solution of $(19)-$ (27)-(28). If $\Phi \in \mathcal{C}^{0}\left([0, T] ; H^{k+3}\left(\Omega_{\mathbf{x}}\right)\right.$, then

$$
\left\|f(t)-f_{h}(t)\right\|_{0, \Omega} \leq C_{b} h^{k+1} \quad \forall t \in[0, T],
$$

where $C_{b}$ depends on the final time $T$, the polynomial degree $k$, the shape regularity of the partition and depends also on $f$ (and therefore on $f_{0}$ ) through the norms

$$
C_{b}=C_{b}\left(\|f(t)\|_{k+2, \Omega},\left\|f_{t}(t)\right\|_{k+1, \Omega},\|f\|_{1, \infty, \Omega},\|\Phi\|_{k+2, \Omega_{\mathbf{x}}},\right.
$$

$$
\left.\|\mathbf{E}\|_{1, \infty, \Omega_{\mathbf{x}}},\|\Phi\|_{2, \infty, \Omega_{\mathbf{x}}}\right) .
$$

We now briefly discuss the above result.

- Unlike what usually happens with the analysis of nonlinear problems, the error estimates given in Theorem 1 are not asymptotic; i.e., they can be guaranteed for any $h<1$. The above theorem is shown without using any a priori assumption made on the discrete solution $\left(\mathbf{E}_{h}, f_{h}\right.$ ) (as it usually happens in the error analysis of non-linear problems). We cope with the nonlinearity by proving an $L^{\infty}$-bound of the approximate electrostatic field and using the assumed regularity of $\mathbf{E}$.

- The optimal rate of convergence for the full DG approximation, requires to approximate the Poisson problem using polynomials one degree higher than the ones used for approximating the distribution function. We also 
note that DG-LDG requires further regularity for the continuous electrostatic field than $R T_{k}$-DG.

- The available existence results for the Vlasov-Poisson system with periodic boundary conditions [9] show the existence of classical solutions, i.e., solutions in $C^{m}(\Omega)$ spaces for all $t \geq 0$, for initial data in $C^{m}(\Omega)$. Note that $C^{m}$-regularity of solutions together with the compact support in velocity imply the regularity assumptions on $f$ and $\Phi$.

As a direct consequence of the previous theorem, we have the following result:

Corollary 1. In the same hypothesis of Theorem 1, let $k \geq 1$. Then:

(a). $\mathbf{R T}_{k}$-DG method: if $\left(\left(\mathbf{E}_{h}, \Phi_{h}\right), f_{h}\right) \in \mathcal{C}^{0}\left([0, T] ;\left(\Sigma_{h}^{k} \times Q_{h}^{k}\right)\right) \times \mathcal{C}^{1}([0, T]$; $\left.\mathcal{Z}_{h}^{k}\right)$ is the $R T_{k}-D G$ approximation solution of (19)-(24), the following estimates hold

$\left\|\mathbf{E}(t)-\mathbf{E}_{h}(t)\right\|_{H\left(\operatorname{div} ; \Omega_{\mathbf{x}}\right)} \leq C h^{k+1}\|\Phi(t)\|_{k+2, \Omega_{\mathbf{x}}}+C_{a} h^{k+1} \quad \forall t \in[0, T]$,

where $C_{a}$ is the constant in Theorem 1.

(b). DG-DG method: let $r=k+1$ and let $\left(\left(\mathbf{E}_{h}, \Phi_{h}\right), f_{h}\right) \in \mathcal{C}^{0}([0, T]$; $\left.\boldsymbol{\Xi}_{h}^{r} \times Q_{h}^{r}\right) \times \mathcal{C}^{1}\left([0, T] ; \mathcal{Z}_{h}^{k}\right)$ be the $D G-D G$ approximation solution of $(19)$ (27)-(28). If $\Phi \in \mathcal{C}^{0}\left([0, T] ; H^{k+3}\left(\Omega_{\mathbf{x}}\right)\right.$, then

$$
\left|\left(\mathbf{E}(t)-\mathbf{E}_{h}(t), \Phi(t)-\Phi_{h}(t)\right)\right|_{\mathcal{A}} \leq C h^{k+1}\|\Phi(t)\|_{k+2, \Omega_{\mathbf{x}}}+C_{b} h^{k+1} \quad \forall t \in[0, T],
$$

where $C_{b}$ is the same constant as in Theorem 1.

The proof of the above corollary follows straightforwardly by substituting the error estimates for the distribution function given in Theorem 1 into the approximation results of Lemmas 3 and 5, stated in $\S 3$.

The rest of the section is devoted to prove Theorem 1 . We start by deriving the error equation and introducing some special projection operators that will be used in our analysis. We then show some auxiliary lemmas and finally, at the very end of the section, we give the proof of the theorem.

4.2. Error Equation and Special Projection Operators. Notice that the solution $(\mathbf{E}, f)$ to $(1)-(3)$ satisfies the variational formulation:

$$
\begin{aligned}
& 0=\sum_{R \in \mathcal{T}_{h}} \int_{R} \frac{\partial f}{\partial t} \varphi_{h} d \mathbf{v} d \mathbf{x}-\int_{R} f \mathbf{v} \cdot \nabla_{x} \varphi_{h} d \mathbf{v} d \mathbf{x}+\int_{R} f \mathbf{E} \cdot \nabla_{\mathbf{v}} \varphi_{h} d \mathbf{v} d \mathbf{x} \\
& +\sum_{T^{v} \in \mathcal{T}_{h_{v}}^{v}} \int_{T^{v}} \int_{\mathcal{E}_{x}}\{\mathbf{v} f\} \cdot \llbracket \varphi_{h} \rrbracket d s_{x} d \mathbf{v}-\sum_{T^{x} \in \mathcal{T}_{h_{x}}^{x}} \int_{T^{x}} \int_{\mathcal{E}_{v}}\{\mathbf{E} f\} \cdot \llbracket \varphi_{h} \rrbracket d s_{v} d \mathbf{x} \quad \forall \varphi_{h} \in \mathcal{Z}_{h}^{k}
\end{aligned}
$$

where we have allowed for a discontinuous test function. Then substracting (19) from above equation we have,

$$
a\left(f-f_{h}, \varphi_{h}\right)+\mathcal{N}\left(\mathbf{E} ; f, \varphi_{h}\right)-\mathcal{N}^{h}\left(\mathbf{E}_{h} ; f_{h}, \varphi_{h}\right)=0 \quad \forall \varphi_{h} \in \mathcal{Z}_{h},
$$


where $a(\cdot, \cdot)$ gathers the linear terms

$a\left(f_{h}, \varphi_{h}\right)=\int_{\Omega}\left(f_{h}\right)_{t} \varphi_{h} d \mathbf{x} d \mathbf{v}-\int_{\Omega} f_{h} \mathbf{v} \cdot \nabla_{\mathbf{x}}^{h} \varphi_{h} d \mathbf{x} d \mathbf{v}+\sum_{T^{v} \in \mathcal{T}_{h_{v}}^{v}} \int_{T^{v}} \int_{\mathcal{E}_{x}}\left\{\mathbf{v} f_{h}\right\}_{\alpha} \llbracket \varphi \rrbracket d s_{\mathbf{x}} d \mathbf{v}$ and $\mathcal{N}^{h}\left(\mathbf{E}_{h} ; \cdot, \cdot\right)(\operatorname{resp} . \mathcal{N}(\mathbf{E} ; \cdot, \cdot))$ carries the nonlinear part;

$$
\begin{aligned}
\mathcal{N}^{h}\left(\mathbf{E}_{h} ; f_{h}, \varphi_{h}\right) & =\int_{\Omega} f_{h} \mathbf{E}_{h} \cdot \nabla_{\mathbf{v}}^{h} \varphi_{h} d \mathbf{v} d \mathbf{x}-\sum_{T^{x} \in \mathcal{T}_{h_{x}}^{x}} \int_{T^{x}} \int_{\mathcal{E}_{v}}\left\{\mathbf{E}_{h} f_{h}\right\}_{\beta} \llbracket \varphi_{h} \rrbracket d s_{\mathbf{v}} d \mathbf{x} \\
\mathcal{N}\left(\mathbf{E} ; f, \varphi_{h}\right) & =\int_{\Omega} f \mathbf{E} \cdot \nabla_{\mathbf{v}}^{h} \varphi_{h} d \mathbf{v} d \mathbf{x}-\sum_{T^{x} \in \mathcal{T}_{h_{x}}^{x}} \int_{T^{x}} \int_{\mathcal{E}_{v}}\{\mathbf{E} f\} \llbracket \varphi_{h} \rrbracket d s_{\mathbf{v}} d \mathbf{x} .
\end{aligned}
$$

We next introduce some special projection operators that will play a crucial role in our error analysis. These projections extend those considered in [6] to the multidimensional case. (See Remark 2 for further comments on the motivation and origin of the projections.) Their definition is based on the use of the one-dimensional projection operators used in [39], that we recall next. Assume $I_{h}=\left\{I_{i}\right\}_{i}$ is FE partition of the unit interval and let denote by $S_{h}^{k}$ the discontinuous finite element space of degree $k$ associated to that partition. Let $\pi^{ \pm}: H^{1 / 2+\epsilon}(I) \longrightarrow S_{h}^{k}$ be the projection operators defined by:

$$
\int_{I_{i}}\left(\pi^{ \pm}(w)-w\right) q_{h} d x=0, \quad \forall q_{h} \in \mathbb{P}_{h}^{k-1}\left(I_{i}\right), \quad \forall i
$$

together with the matching conditions;

$$
\pi^{+}\left(w\left(x_{i-1 / 2}^{+}\right)\right)=w\left(x_{i-1 / 2}^{+}\right) ; \quad \pi^{-}\left(w\left(x_{i+1 / 2}^{-}\right)\right)=w\left(x_{i+1 / 2}^{-}\right) .
$$

Notice that more regularity than $L^{2}(I)$ is required for defining these projections. The following error estimates can be easily shown for all these projections:

$$
\left\|w-\pi^{ \pm}(w)\right\|_{0, I_{i}} \leq C h^{k+1}|w|_{k+1, I_{i}} \quad \forall w \in H^{k+1}\left(I_{i}\right)
$$

where $C$ is a constant depending only on the shape-regularity of the mesh and the polynomial degree [20,39].

We denote by $\Pi_{h}: \mathcal{C}^{0}(\Omega) \longrightarrow \mathcal{Z}_{h}^{k}$ the projection operator defined as follows: Let $R=T^{x} \times T^{v}$ be an arbitrary element of $\mathcal{T}_{h}$ and let $w \in \mathcal{C}^{0}(\bar{R})$. The restriction of $\Pi_{h}(w)$ to $R$ is defined by:

$$
\Pi_{h}(w)= \begin{cases}\left(\widetilde{\Pi}_{x} \otimes \widetilde{\Pi}_{v}\right)(w) & \text { if } \operatorname{sign}(\mathbf{E} \cdot \mathbf{n})=\text { constant } \\ \left(\widetilde{\Pi}_{x} \otimes \widetilde{\mathcal{P}}_{v}\right)(w) & \text { if } \operatorname{sign}(\mathbf{E} \cdot \mathbf{n}) \neq \text { constant }\end{cases}
$$

where $\widetilde{\Pi}_{x}: \mathcal{C}^{0}\left(\Omega_{\mathbf{x}}\right) \longrightarrow X_{h}^{k}$ and $\widetilde{\Pi}_{v}: \mathcal{C}^{0}\left(\Omega_{\mathbf{v}}\right) \longrightarrow V_{h}^{k}$ are the projection operators:

$$
\widetilde{\Pi}_{\mathbf{x}}(w)=\left\{\begin{array}{ll}
\Pi_{\mathbf{x}}^{-}(w) & \text { if } \mathbf{v} \cdot \mathbf{n}_{\left.\right|_{\partial T^{x}} ^{-}}^{-}>0, \\
\Pi_{\mathbf{x}}^{+}(w) & \text { if } \mathbf{v} \cdot \mathbf{n}_{\left.\right|_{\partial T^{x}}}^{-}<0,
\end{array} \quad \widetilde{\Pi}_{\mathbf{v}}(w)= \begin{cases}\Pi_{\mathbf{v}}^{+}(w) & \text { if } \mathbf{E} \cdot \mathbf{n}_{\left.\right|_{\partial T^{v}} ^{-}}^{-}>0 \\
\Pi_{\mathbf{v}}^{-}(w) & \text { if } \mathbf{E} \cdot \mathbf{n}_{\left.\right|_{\partial T^{v}}}^{-}<0\end{cases}\right.
$$


and $\Pi_{s}^{ \pm}$with $s=\mathbf{x}$ or $\mathbf{v}$ are defined as the tensor product of the one-dimensional projections $\pi^{ \pm}$given in (35)-(36):

(39)

$\Pi_{s}^{ \pm}=\pi_{s, 1}^{ \pm} \times \pi_{s, 2}^{ \pm} \quad$ for $d=2, \quad \Pi_{s}^{ \pm}=\pi_{s, 1}^{ \pm} \times \pi_{s, 2}^{ \pm} \otimes \pi_{s, 3}^{ \pm} \quad$ for $d=3, \quad s=\mathbf{x}$ or $\mathbf{v}$.

In the above definition, the subscript $i$ in $\pi_{x, i}^{ \pm}$and $\pi_{v, i}^{ \pm}$refers to the fact that projection is along the $i$-th direction (component) in the $\mathbf{x}$ and $\mathbf{v}$ "spaces", respectively.

To complete the definition of the projection $\Pi_{h}$ we need to provide the definition of $\widetilde{\mathcal{P}}_{v}: L^{2}\left(\Omega_{\mathbf{v}}\right) \longrightarrow V_{h}^{k}$, which accounts for the cases where $\mathbf{E} \cdot \mathbf{n}_{\left.\right|_{\partial T^{v}}}^{-}$changes sign across any single $(2 d-1)$-element $e \subset T^{x} \times \partial T^{v}$. From the structure of the partition such condition amounts to have at least one of the components of $\mathbf{E}$ vanishing within the element $R$ (and so in $T^{x}$ ). For simplicity, we give the detailed definition in the case $d=2$ (the case $d=3$ is similar but taking into account more cases). Let $\mathbf{E}=\left[E_{1}, E_{2}\right]^{t}$, then

(40)

$$
\widetilde{\mathcal{P}}_{v}(w)= \begin{cases}{\left[\mathcal{P}_{v, 1} \otimes \tilde{\pi}_{v, 2}\right](w)} & \text { if } \operatorname{sign}\left(E_{1}\right) \neq \text { constant \& } \operatorname{sign}\left(E_{2}\right)=\text { constant } \\ {\left[\tilde{\pi}_{v, 1} \otimes \mathcal{P}_{v, 2}\right](w)} & \text { if } \operatorname{sign}\left(E_{1}\right)=\text { constant \& } \operatorname{sign}\left(E_{2}\right) \neq \text { constant } \\ {\left[\mathcal{P}_{v, 1} \otimes \mathcal{P}_{v, 2}\right](w)} & \text { if } \operatorname{sign}\left(E_{1}\right) \neq \text { constant \& } \operatorname{sign}\left(E_{2}\right) \neq \text { constant }\end{cases}
$$

Here, $\mathcal{P}_{v, i}, i=1,2$ stands for the standard one-dimensional projection along the $v_{i}$ direction. With a small abuse in the notation we have denoted by $\tilde{\pi}_{v, j}=\pi_{v, j}^{ \pm}$, $j=1,2$ where the + and - signs refer to whether $\mathbf{E} \cdot \mathbf{n}= \pm E_{j}$ is positive or negative. Note that this is consistent with the definition of $\widetilde{\Pi}_{v}$ given in (38).

Observe that conditions (37)-(38)-(39)-(40) together with (35)-(36), define the projection $\Pi_{h}(w)$ uniquely for any given $w \in \mathcal{C}^{0}(\Omega)$. This projection, $\Pi_{h}$, is nothing but the extension to higher dimension $2 d, d=2,3$ of the projection used in [6]. See Remarks 3 and 2 for further comments.

Remark 2. The definition of $\Pi_{h}$ is inspired in those introduced in the two dimensional case, for a linear transport equation in [33] and for a Poisson problem in [21]. In fact, in [33], the authors display the error analysis by using an "(interpolation) operator" that in each element (a rectangle or square), reproduces the value of the interpolated function at the Gauss-Radau nodes. To the best of our knowledge, this idea was first coded in terms of projection operators in [21]. Notice that the property of collocation at one boundary end of $\pi^{ \pm}$given in (36) is just reflecting the fact that of using Gauss-Radau nodes for the interpolation operator. This is indeed the essential feature required in the proof of Lemma 12 (given in Appendix B), which allows for proving optimal approximation properties for $\mathcal{K}^{1}$ and $\mathcal{K}^{2}$ (defined in (45)-(46)-(47)), and in turn will allow for achieving optimal rate of convergence.

Next lemma, although elementary, provides the basic approximation properties we need in our analysis. 
Lemma 7. Let $w \in H^{s+2}(R), s \geq 0$ and let $\Pi_{h}$ be the projection operator defined through (37)-(38). Then,

(41)

$$
\begin{aligned}
\left\|w-\Pi_{h}(w)\right\|_{0, R} & \leq C h^{\min (s+2, k+1)}\|w\|_{s+1, R}, \\
\left\|w-\Pi_{h}(w)\right\|_{0, e} & \leq C h^{\min \left(s+\frac{3}{2}, k+\frac{1}{2}\right)}\|w\|_{s+1, R}, \quad \forall e \subset \partial T^{x} \times T^{v}, \quad e \subset T^{x} \times \partial T^{v} .
\end{aligned}
$$

Proof. From the definition (37) we distinguish two cases. If $R=T^{x} \times T^{v}$ is an element where $\mathbf{E}(\mathbf{x}) \cdot \mathbf{n}$ does not change sign inside $e \subset T^{x} \times \partial T^{v}$, the proof is the same as [18, Lemma 3.2]. If on the contrary, $T^{x}$ is such that $\exists \mathbf{x}^{*} \in T^{x}$ for which $\mathbf{E}\left(\mathbf{x}^{*}\right) \cdot \mathbf{n}=0$ at $T^{x} \times \partial T^{v}$, we have $\Pi_{h}(w)=\widetilde{\Pi}_{x} \otimes \widetilde{\mathcal{P}}_{v}(w)$. But still, since $\Pi_{h}$ is a polynomial preserving and linear operator, estimates (41) follow also in this case from Bramble-Hilbert lemma, trace Theorem and standard scaling arguments. Details are omitted for the sake of conciseness.

Summing estimates (41) from Lemma 7 , over elements of the partition $\mathcal{T}_{h}$, we have

$$
\begin{array}{r}
\left\|w-\Pi_{h}(w)\right\|_{0, \mathcal{T}_{h}}+h^{-1 / 2}\left\|w-\Pi_{h}(w)\right\|_{0, \mathcal{E}_{\mathbf{x}} \times \mathcal{T}_{h_{v}}^{v}}+h^{-1 / 2}\left\|w-\Pi_{h}(w)\right\|_{0, \mathcal{T}_{h_{x}}^{x} \times \mathcal{E}_{\mathbf{v}}} \\
\leq C h^{k+1}\|w\|_{k+1, \Omega}
\end{array}
$$

Next, we write

$$
f-f_{h}=\left[\Pi_{h}(f)-f_{h}\right]-\left[\Pi_{h}(f)-f\right]=\omega^{h}-\omega^{e} .
$$

Taking now as test function $\varphi_{h}=\omega^{h} \in \mathcal{Z}_{h}^{k}$, the error equation (34) becomes

$$
a\left(\omega^{h}-\omega^{e}, \omega^{h}\right)+\mathcal{N}\left(\mathbf{E} ; f, \omega^{h}\right)-\mathcal{N}^{h}\left(\mathbf{E}_{h} ; f_{h}, \omega^{h}\right)=0 .
$$

Finally, we define

$$
\mathcal{K}^{1}\left(\mathbf{v}, f, \omega^{h}\right)=\sum_{\mathcal{R} \in \mathcal{T}_{h}} \mathcal{K}_{\mathcal{R}}^{1}\left(\mathbf{v}, f, \omega^{h}\right), \quad \mathcal{K}^{2}\left(\mathbf{E}_{h}, f, \omega^{h}\right)=\sum_{\mathcal{R} \in \mathcal{T}_{h}} \mathcal{K}_{\mathcal{R}}^{2}\left(\mathbf{E}_{h}, f, \omega^{h}\right)
$$

where

$$
\begin{aligned}
\mathcal{K}_{\mathcal{R}}^{1}\left(\mathbf{v}, f, \omega^{h}\right) & =\int_{\mathcal{R}} \omega^{e} \mathbf{v} \cdot \nabla_{\mathbf{x}} \omega^{h} d \mathbf{x} d \mathbf{v}-\int_{T^{v}} \int_{\partial T^{x}}\left(\widehat{\mathbf{v} \cdot \mathbf{n} \omega^{e}}\right) \omega^{h} d \mathbf{v} d s_{\mathbf{x}} \\
\mathcal{K}_{\mathcal{R}}^{2}\left(\mathbf{E}_{h}, f, \omega^{h}\right) & =\int_{\mathcal{R}} \omega^{e} \mathbf{E}_{h} \cdot \nabla_{\mathbf{v}} \omega^{h} d \mathbf{x} d \mathbf{v}-\int_{T^{x}} \int_{\partial T^{v}}\left(\widehat{\mathbf{E}_{h} \cdot \mathbf{n} \omega^{e}}\right) \omega^{h} d \mathbf{x} d s_{\mathbf{v}} .
\end{aligned}
$$

The next two lemmas provide some estimates for the two expressions defined in (45). The proof of these two results are the extension to the higher dimensional case of [6, Lemma 4.5] and [6, Lemma 4.6], respectively. Their proofs are given in Appendix B.

Lemma 8. Let $\mathcal{T}_{h}=\mathcal{T}_{h_{x}}^{x} \times \mathcal{T}_{h_{v}}^{v}$ be the tensor product of two cartesian meshes $\mathcal{T}_{h_{x}}^{x}$ and $\mathcal{T}_{h_{v}}^{v}$ of $\Omega_{\mathbf{x}}$ and $\Omega_{\mathbf{v}}$, respectively. Let $k \geq 1$ and let $f \in \mathcal{C}^{0}([0, T]$; $\left.W^{1, \infty}(\Omega) \times H^{k+2}(\Omega)\right)$ be the distribution function solution of (1)-(3). Let $f_{h} \in \mathcal{Z}_{h}^{k}$ 
be its approximation satisfying (16) and let $\mathcal{K}^{1}$ be defined as in (45)-(46). Assume that the partition $\mathcal{T}_{h}$ is constructed so that none of the components of $\mathbf{v}$ vanish inside any element. Then, the following estimate hold

$$
\left|\mathcal{K}^{1}\left(\mathbf{v}, f, \omega^{h}\right)\right| \leq C h^{k+1}\left(\|f\|_{k+1, \Omega}+C L\|f\|_{k+2, \Omega}\right)\left\|\omega^{h}\right\|_{0, \mathcal{T}_{h}} .
$$

Lemma 9. Let $\mathcal{T}_{h}$ be a cartesian mesh of $\Omega, k \geq 1$ and let $\left(\mathbf{E}_{h}, f_{h}\right) \in \widetilde{\boldsymbol{\Sigma}}_{h} \times \mathcal{Z}_{h}^{k}$ be the solution to (19) with either $\widetilde{\boldsymbol{\Sigma}}_{h}=\boldsymbol{\Sigma}_{h}^{r}$ or $\widetilde{\boldsymbol{\Sigma}}_{h}=\boldsymbol{\Xi}_{h}^{r}, r \geq 1$. Let $(\mathbf{E}, f) \in$ $\mathcal{C}^{0}\left([0, T] ; W^{1, \infty}(\Omega) \times H^{k+2}(\Omega)\right)$ and let $\mathcal{K}^{2}$ be defined as in (45)-(47). Then, the following estimate holds

$$
\begin{aligned}
\left|\mathcal{K}^{2}\left(\mathbf{E}_{h}, f, \omega^{h}\right)\right| \leq & C h^{k}\left\|\mathbf{E}-\mathbf{E}_{h}\right\|_{0, \infty, \mathcal{T}_{h x}^{x}}\|f\|_{k+1, \Omega}\left\|\omega^{h}\right\|_{0, \mathcal{T}_{h}} \\
& +C h^{k+1}\left(\|f\|_{k+2, \Omega}\|\mathbf{E}\|_{0, \infty, \Omega_{\mathbf{x}}}+\|f\|_{k+1, \Omega}|\mathbf{E}|_{1, \infty, \Omega_{\mathbf{x}}}\right)\left\|\omega^{h}\right\|_{0, \mathcal{T}_{h}} .
\end{aligned}
$$

Remark 3. We wish to note that, as it happens for $d=1[6]$, the definition (37) of $\Pi_{h}$ is done in terms of $\mathbf{E}$ (and $\mathbf{v}$ ), while the definition of the numerical fluxes is done in terms of $\mathbf{E}_{h}$ (and $\mathbf{v}$ ). This is due to the non-linearity of the problem and it is inspired in the ideas used in [40]. By defining $\Pi_{h}$ in terms of $\mathbf{E}$ rather than $\mathbf{E}_{h}$ and using the regularity of the solution, one can estimate optimally the expression $\mathcal{K}^{2}$ without any further assumption on the mesh partition $\mathcal{T}_{h}$.

4.3. Auxiliary Results. We next give two auxiliary results that will be required for our subsequent analysis.

Lemma 10. Let $f \in \mathcal{C}^{0}(\Omega)$ and let $f_{h} \in \mathcal{Z}_{h}^{k}$ with $k \geq 0$. Then, the following equality holds true,

$$
\begin{aligned}
a\left(f-f_{h}, \omega^{h}\right)= & \sum_{\mathcal{R} \in \mathcal{T}_{h}} \int_{\mathcal{R}}\left(\omega_{t}^{h}-\omega_{t}^{e}\right) \omega^{h} d \mathbf{x} d \mathbf{v} \\
& +\sum_{T^{v} \in \mathcal{T}_{h_{v}}^{v}} \int_{T^{v}} \int_{\mathcal{E}_{x}} \frac{|\mathbf{v} \cdot \mathbf{n}|}{2} \llbracket \omega^{h} \rrbracket^{2} d s_{x} d \mathbf{v}+\mathcal{K}^{1}\left(\mathbf{v}, f, \omega^{h}\right) .
\end{aligned}
$$

Proof. Noting that

$$
a\left(f-f_{h}, \omega^{h}\right)=a\left(\omega^{h}, \omega^{h}\right)-a\left(\omega^{e}, \omega^{h}\right) .
$$

The first term is readily estimated arguing as in the proof of Proposition 1

$$
a\left(\omega^{h}, \omega^{h}\right)=\sum_{\mathcal{R} \in \mathcal{T}_{h}} \int_{\mathcal{R}} \omega_{t}^{h} \omega^{h} d \mathbf{x} d \mathbf{v}+\sum_{T^{v} \in \mathcal{T}_{h_{v}}^{v}} \int_{T^{v}} \int_{\mathcal{E}_{x}^{0}} \frac{|\mathbf{v} \cdot \mathbf{n}|}{2} \llbracket \omega^{h} \rrbracket^{2} d s_{x} d \mathbf{v}
$$


For the second term, using the continuity of $f$ and the consistency of the numerical fluxes (17) and recalling the definition (45), we easily get

$$
\begin{aligned}
a\left(\omega^{e}, \omega^{h}\right)= & \sum_{\mathcal{R} \in \mathcal{T}_{h}} \int_{\mathcal{R}} \omega_{t}^{e} \omega^{h} d \mathbf{x} d \mathbf{v}-\int_{\Omega} \omega^{e} \mathbf{v} \cdot \nabla_{\mathbf{x}}^{h} \omega^{h} d \mathbf{x} d \mathbf{v} \\
& +\sum_{T^{v} \in \mathcal{T}_{h_{v}}^{v}} \int_{T^{v}} \int_{\mathcal{E}_{x}}\left\{\mathbf{v} \omega^{e}\right\}_{\alpha} \cdot \llbracket \omega^{h} \rrbracket d s_{x} d \mathbf{v} \\
= & \sum_{\mathcal{R} \in \mathcal{T}_{h}} \int_{\mathcal{R}} \omega_{t}^{e} \omega^{h} d \mathbf{x} d \mathbf{v}-\mathcal{K}^{1}\left(\mathbf{v}, f, \omega^{h}\right),
\end{aligned}
$$

which concludes the proof.

The other auxiliary lemma deals with the error coming from the nonlinear term:

Lemma 11. Let $\mathbf{E} \in \mathcal{C}^{0}\left(\Omega_{\mathbf{x}}\right), f \in \mathcal{C}^{0}(\Omega)$ and $f_{h} \in \mathcal{Z}_{h}^{k}$ with $k \geq 0$. Then, the following identity holds true,

$$
\begin{aligned}
\mathcal{N}\left(\mathbf{E} ; f ; \omega^{h}\right)-\mathcal{N}^{h}\left(\mathbf{E}_{h} ; f_{h}, \omega^{h}\right)= & \sum_{T^{x} \in \mathcal{T}_{h x}^{x}} \int_{T^{x}} \int_{\mathcal{E}_{v}^{0}} \frac{\left|\mathbf{E}_{h} \cdot \mathbf{n}\right|}{2} \llbracket \omega^{h} \rrbracket^{2} d s_{v} d \mathbf{x} \\
& -\int_{\Omega}\left[\mathbf{E}-\mathbf{E}_{h}\right] \cdot \nabla_{\mathbf{v}}^{h} f \omega^{h} d \mathbf{v} d \mathbf{x}-\mathcal{K}^{2}\left(\mathbf{E}_{h}, f, \omega^{h}\right) .
\end{aligned}
$$

Proof. Subtracting the discrete and continuous nonlinear terms, using the continuity of $\mathbf{E}$ and $f$, the consistency of the numerical flux $\widehat{\mathbf{E}_{h} f_{h}}$ together with (9), we find

$$
\begin{aligned}
\mathcal{N}\left(\mathbf{E} ; f ; \omega^{h}\right) & -\mathcal{N}^{h}\left(\mathbf{E}_{h} ; f_{h}, \omega^{h}\right)=\int_{\Omega}\left[f \mathbf{E}-f_{h} \mathbf{E}_{h}\right] \cdot \nabla_{\mathbf{v}}^{h} \omega^{h} d \mathbf{v} d \mathbf{x} \\
& -\sum_{T^{x} \in \mathcal{T}_{h_{x}}^{x}} \int_{T^{x}} \int_{\mathcal{E}_{v}}\left\{\mathbf{E} f-\mathbf{E}_{h} f_{h}\right\}_{\beta} \cdot \llbracket \omega^{h} \rrbracket d s_{v} d \mathbf{x}=T_{1}+T_{2}+T_{3},
\end{aligned}
$$

where in the last step we have decomposed the integrand of the volume part as

$$
\mathbf{E} f-\mathbf{E}_{h} f_{h}=\left(\mathbf{E}-\mathbf{E}_{h}\right) f+\mathbf{E}_{h}\left(f-f_{h}\right),
$$

so that,

$$
\begin{aligned}
& T_{1}=\int_{\Omega} f\left[\mathbf{E}-\mathbf{E}_{h}\right] \cdot \nabla_{v}^{h} \omega^{h} d \mathbf{v} d \mathbf{x}, \\
& T_{2}=\int_{\Omega}\left[f-f_{h}\right] \mathbf{E}_{h} \cdot \nabla_{v}^{h} \omega^{h} d \mathbf{v} d \mathbf{x}, \\
& T_{3}=\sum_{T^{x} \in \mathcal{T}_{h_{x}}^{x}} \int_{T^{x}} \int_{\mathcal{E}_{v}^{0}}\left\{\mathbf{E}_{h} f_{h}-\mathbf{E} f\right\}_{\beta} \cdot \llbracket \omega^{h} \rrbracket d s_{v} d \mathbf{x} .
\end{aligned}
$$


Integrating by parts $T_{1}$ and using the continuity of $f$ together with (9) and the fact that neither $\mathbf{E}$ nor $\mathbf{E}_{h}$ depend on $v$, we have

$$
T_{1}=-\int_{\Omega}\left[\mathbf{E}-\mathbf{E}_{h}\right] \cdot \nabla_{v}^{h} f \omega^{h} d \mathbf{v} d \mathbf{x}+\sum_{T^{x} \in \mathcal{T}_{h_{x}}^{x}} \int_{T^{x}} \int_{\mathcal{E}_{v}}\left\{\mathbf{E}-\mathbf{E}_{h}\right\} \cdot \llbracket \omega^{h} \rrbracket f d s_{v} d \mathbf{x}=T_{1 a}+T_{1 b} .
$$

We next deal with $T_{2}$. From the splitting (43), direct integration and (9), we get

$$
\begin{aligned}
T_{2} & =\frac{1}{2} \int_{\Omega} \mathbf{E}_{h} \cdot \nabla_{v}^{h}\left(\omega^{h}\right)^{2} d \mathbf{v} d \mathbf{x}-\int_{\Omega} \omega^{e} \mathbf{E}_{h} \cdot \nabla_{v}^{h} \omega^{h} d \mathbf{v} d \mathbf{x} \\
& =\frac{1}{2} \sum_{T^{x} \in \mathcal{T}_{h_{x}}^{x}} \int_{T^{x}} \int_{\mathcal{E}_{v}}\left\{\mathbf{E}_{h}\right\} \cdot \llbracket\left(\omega^{h}\right)^{2} \rrbracket d s_{v} d \mathbf{x}-\int_{\Omega} \omega^{e} \mathbf{E}_{h} \cdot \nabla_{v}^{h} \omega^{h} d \mathbf{v} d \mathbf{x}=T_{2 a}+T_{2 b} .
\end{aligned}
$$

We finally deal with the boundary terms collected in $T_{3}$. Reasoning as in (51) and using the continuity of $f$ together with the consistency of the numerical flux $\widehat{\mathbf{E}_{h} f_{h}}$, we find

$$
\begin{aligned}
T_{3} & =\sum_{T^{x} \in \mathcal{T}_{h_{x}}^{x}} \int_{T^{x}} \int_{\mathcal{E}_{v}}\left[\left\{\left(\mathbf{E}_{h}-\mathbf{E}\right) f\right\} \cdot \llbracket \omega^{h} \rrbracket-\left\{\mathbf{E}_{h} \omega^{h}\right\}_{\beta} \cdot \llbracket \omega^{h} \rrbracket+\left\{\mathbf{E}_{h} \omega^{e}\right\}_{\beta} \cdot \llbracket \omega^{h} \rrbracket\right] d s_{v} d \mathbf{x} \\
& =T_{3 a}+T_{3 b}+T_{3 c} .
\end{aligned}
$$

The first term above, $T_{3 a}$, cancels with $T_{1 b}$ in (52). Arguing as in Proposition 1, the sum of the second term above $T_{3 b}$ and $T_{2 a}$ (53) gives

$$
T_{3 b}+T_{2 a}=\sum_{T^{x} \in \mathcal{T}_{h x}^{x}} \int_{T^{x}} \int_{\mathcal{E}_{v}} \frac{\left|\mathbf{E}_{h} \cdot \mathbf{n}\right|}{2} \llbracket \omega^{h} \rrbracket^{2} d s_{v} d \mathbf{x}
$$

Finally, recalling the definition (46) we have

$$
T_{2 b}+T_{3 c}=-\mathcal{K}^{2}\left(\mathbf{E}_{h}, f, \omega^{h}\right),
$$

and so substituting in (50) the above results together with $T_{1 a}$ the proof is completed.

We have now all ingredients to carry out the proof of Theorem 1 . 


\subsection{Proof of Theorem 1.}

Proof. Substituting in the error equation (44) the expressions from Lemmas 10 and 11 and using standard triangle inequality, we find

$$
\begin{aligned}
& \frac{d}{d t}\left\|\omega^{h}\right\|_{0, \mathcal{T}_{h}}^{2}+\frac{1}{2}\left\||\mathbf{v} \cdot \mathbf{n}|^{1 / 2} \llbracket \omega^{h} \rrbracket\right\|_{\mathcal{E}_{x} \times \mathcal{T}_{h v}^{v}}^{2}+\frac{1}{2}\left\|\left|\mathbf{E}_{h} \cdot \mathbf{n}\right|^{1 / 2} \llbracket \omega^{h} \rrbracket\right\|_{\mathcal{T}_{h_{x}}^{x} \times \mathcal{E}_{v}}^{2} \\
& =\sum_{\mathcal{R} \in \mathcal{T}_{h}} \int_{\mathcal{R}} \omega_{t}^{e} \omega^{h} d \mathbf{x} d \mathbf{v}+\int_{\Omega}\left[\mathbf{E}-\mathbf{E}_{h}\right] \cdot \nabla_{\mathbf{v}} f \omega^{h} d \mathbf{v} d \mathbf{x}-\mathcal{K}^{1}\left(\mathbf{v}, f, \omega^{h}\right)+\mathcal{K}^{2}\left(\mathbf{E}_{h}, f, \omega^{h}\right) \\
& =I_{1}+I_{2}-\mathcal{K}^{1}+\mathcal{K}^{2} \leq\left|I_{1}\right|+\left|I_{2}\right|+\left|\mathcal{K}^{1}\right|+\left|\mathcal{K}^{2}\right| .
\end{aligned}
$$

The first and third term are independent of the approximation to the electrostatic field $\mathbf{E}_{h}$ and therefore are estimated in the same way for both cases (a) and (b).

For the first term, Cauchy-Schwarz and the arithmetic-geometric inequality together with the approximation estimate (42) give

$$
\left|I_{1}\right| \leq C h^{2 k+2}\left\|f_{t}\right\|_{k+1, \Omega}^{2}+C\left\|\omega^{h}\right\|_{0, \mathcal{T}_{h}}^{2} .
$$

Third term is estimated by means of estimate (48) from Lemma 8 and the arithmetic-geometric inequality,

$$
\left|\mathcal{K}^{1}\right| \leq C h^{2 k+2}\left(\|f\|_{k+1, \Omega}+C L\|f\|_{k+2, \Omega}\right)^{2}+C\left\|\omega^{h}\right\|_{0, \mathcal{T}_{h}}^{2} .
$$

Next we estimate the second and fourth terms in (54), which depend on the approximation to the electrostatic field. We first deal with the $R T_{k}$-DG method (case (a)). Hölder inequality, the arithmetic-geometric inequality and estimate (25) from Lemma 3 together with the approximation estimate (42), give for the second term

$$
\begin{aligned}
\left|I_{2}\right| \leq & C\left\|\mathbf{E}-\mathbf{E}_{h}\right\|_{0, \Omega_{x}}^{2}\left\|\nabla_{v} f\right\|_{0, \infty, \Omega}+C\left\|\nabla_{v} f\right\|_{0, \infty, \Omega}\left\|\omega^{h}\right\|_{0, \mathcal{T}_{h}}^{2} \\
\leq & C h^{2 k+2}\|f\|_{1, \infty, \Omega}\left[\left(\|\mathbf{E}(t)\|_{k+1, \Omega_{\mathbf{x}}}+\|\Phi\|_{k+2, \Omega_{\mathbf{x}}}\right)^{2}+C\|f\|_{k+1, \Omega}^{2}\right] \\
& +2 C\|f\|_{1, \infty, \Omega}\left\|\omega^{h}\right\|_{0, \mathcal{T}_{h}}^{2} .
\end{aligned}
$$

To deal with the last term, we observe that the bound (49) in Lemma 9

$\left|\mathcal{K}^{2}\right| \leq C h^{k}\left\|\mathbf{E}-\mathbf{E}_{h}\right\|_{0, \infty, \mathcal{T}_{h x}^{x}}\|f\|_{k+1, \Omega}\left\|\omega^{h}\right\|_{0, \mathcal{T}_{h}}+C h^{k+1}\|f\|_{k+2, \Omega}\|\mathbf{E}\|_{1, \infty, \Omega_{\mathbf{x}}}\left\|\omega^{h}\right\|_{0, \mathcal{T}_{h}}$,

requires an $L^{\infty}$-bound on the error $\mathbf{E}-\mathbf{E}_{h}$. This is obtained by combining estimate (26) from Lemma 4 with the bound (22) from Lemma 2 and the approximation property (15) for $p=\infty$,

$$
\begin{aligned}
\left\|\mathbf{E}-\mathbf{E}_{h}\right\|_{0, \infty, \Omega_{\mathbf{x}}} \leq & C h\|\mathbf{E}\|_{1, \infty, \Omega_{\mathbf{x}}}+C h^{3 / 2} \mid \log (h)\|\rho\|_{W^{3 / 2, d}\left(\Omega_{\mathbf{x}}\right)} \\
& +C L^{d / 2} h^{1-d / 2}|\log (h)|\left(C h^{k+1}\|f\|_{k+1, \Omega}+\left\|f_{h}-\mathcal{P}_{h}(f)\right\|_{0, \mathcal{T}_{h}}\right) .
\end{aligned}
$$


Notice now that since $\mathcal{P}_{h}$ is polynomial preserving, $\mathcal{P}_{h}[\widetilde{\Pi}(f)]=\widetilde{\Pi}(f)$ and so using also that it is stable in $L^{2}$, we have

$$
\begin{aligned}
\left\|f_{h}-\mathcal{P}_{h}(f)\right\|_{0, \mathcal{T}_{h}} & \leq\left\|f_{h}-\widetilde{\Pi}(f)\right\|_{0, \mathcal{T}_{h}}+\left\|\widetilde{\Pi}(f)-\mathcal{P}_{h}(f)\right\|_{0, \mathcal{T}_{h}} \\
& \leq\left\|f_{h}-\widetilde{\Pi}(f)\right\|_{0, \mathcal{T}_{h}}+C\|\widetilde{\Pi}(f)-f\|_{0, \mathcal{T}_{h}} .
\end{aligned}
$$

Substituting the above estimate into (59) and using the approximation property (42), we find

$$
\begin{aligned}
\left\|\mathbf{E}-\mathbf{E}_{h}\right\|_{0, \infty, \Omega_{\mathbf{x}}} \leq & C h|\mathbf{E}|_{1, \infty, \Omega_{\mathbf{x}}}+C h^{3 / 2}|\log (h)|\|\rho\|_{W^{3 / 2, d}\left(\Omega_{\mathbf{x}}\right)} \\
& \left.+C h^{k+2-d / 2}|\log (h)|\|f\|_{k+1, \Omega}+C h^{1-d / 2}|\log (h)|\left\|\omega^{h}\right\|_{0, \mathcal{T}_{h}}\right) .
\end{aligned}
$$

Plugging now the above result in estimate (58) and using the arithmetic-geometric inequality we finally get for the last term in (54),

$$
\begin{aligned}
\left|K^{2}\right| \leq & C h^{k+1}\left(\|f\|_{k+2, \Omega}\|\mathbf{E}\|_{1, \infty, \Omega_{\mathbf{x}}}\right. \\
& \left.\quad+\|f\|_{k+1, \Omega}\left[|\mathbf{E}|_{1, \infty, \Omega_{\mathbf{x}}}+C h^{1 / 2}|\log (h)|\|\rho\|_{W^{3 / 2, d}\left(\Omega_{\mathbf{x}}\right)}\right]\right)\left\|\omega^{h}\right\|_{0, \mathcal{T}_{h}} \\
& +C h^{2 k+2-d / 2}|\log (h)|\|f\|_{k+1, \Omega}^{2}\left\|\omega^{h}\right\|_{0, \mathcal{T}_{h}}+C h^{k+1-d / 2}|\log (h)|\|f\|_{k+1, \Omega}\left\|\omega^{h}\right\|_{0, \mathcal{T}_{h}}^{2} \\
\leq & C h^{2 k+2}\|f\|_{k+2, \Omega}^{2}\|\mathbf{E}\|_{1, \infty, \Omega_{\mathbf{x}}}^{2}+C h^{4 k+4-d}|\log (h)|^{2}\|f\|_{k+1, \Omega}^{4} \\
& +C\left\|\omega^{h}\right\|_{0, \mathcal{T}_{h}}^{2}+\left(C h|\log (h)|^{2}\|\rho\|_{W^{3 / 2, d}\left(\Omega_{\mathbf{x}}\right)}+C h^{k+1-d / 2}|\log (h)|\|f\|_{k+1, \Omega}\right)\left\|\omega^{h}\right\|_{0, \mathcal{T}_{h}}^{2} .
\end{aligned}
$$

Observe that since $k \geq 1$ the coefficient of the term $\left\|\omega^{h}\right\|_{0, \mathcal{T}_{h}}^{2}$ is uniformly bounded for all $h<1$; i.e., $\exists$ a constant $c_{1}>0$ independent of $h$ such that

$$
\begin{aligned}
C\left\|\omega^{h}\right\|_{0, \mathcal{T}_{h}}^{2} & +\left(C h|\log (h)|^{2}\|\rho\|_{W^{3 / 2, d}\left(\Omega_{\mathbf{x}}\right)}\right. \\
& \left.+C h^{k+1-d / 2}|\log (h)|\|f\|_{k+1, \Omega}\right)\left\|\omega^{h}\right\|_{0, \mathcal{T}_{h}}^{2} \leq c_{1}\left\|\omega^{h}\right\|_{0, \mathcal{T}_{h}}^{2} .
\end{aligned}
$$

Hence,

$$
\left|\mathcal{K}^{2}\right| \leq C h^{2 k+2}\left(\|f\|_{k+2, \Omega}^{2}\|\mathbf{E}\|_{1, \infty, \Omega_{\mathbf{x}}}^{2}+L^{d / 2}\|f\|_{k+1, \Omega}^{2}\right)+c_{1}\left\|\omega^{h}\right\|_{0, \mathcal{T}_{h}}^{2},
$$

where we have already discarded the higher order terms. Now, substituting into (54) the above estimate together with (55), (57) and (56), we obtain

$$
\begin{gathered}
\frac{d}{d t}\left\|\omega^{h}\right\|_{0, \mathcal{T}_{h}}^{2}+\frac{1}{2}\left\|\left|\mathbf{v} \cdot \mathbf{n}^{-}\right|{ }^{1 / 2} \llbracket \omega^{h} \rrbracket\right\|_{\mathcal{E}_{x}^{0} \times \mathcal{T}_{h v}^{v}}^{2}+\frac{1}{2}\left\|\left|\mathbf{E}_{h} \cdot \mathbf{n}^{-}\right|^{1 / 2} \llbracket \omega^{h} \rrbracket\right\|_{\mathcal{T}_{h x}^{x} \times \mathcal{E}_{v}^{0}}^{2} \\
\leq C h^{2 k+2}\left[\|f\|_{k+2, \Omega}^{2}\left(\|\mathbf{E}\|_{1, \infty}^{2}+C L^{d / 2}\right)+\left\|f_{t}\right\|_{k+1, \Omega}^{2}\right. \\
\left.+\|f\|_{1, \infty, \Omega}\left(\|\mathbf{E}(t)\|_{k+1, \Omega_{\mathbf{x}}}+\|\Phi\|_{k+2, \Omega_{\mathbf{x}}}\right)^{2}\right] \\
+\left(c_{1}+2 C\|f\|_{1, \infty, \Omega}+C\right)\left\|\omega^{h}\right\|_{0, \mathcal{T}_{h}}^{2} .
\end{gathered}
$$

Integrating in time the above inequality together with a standard application of Gronwall's inequality ([32]) gives the error estimate,

$$
\left\|\omega^{h}(t)\right\|_{0, \mathcal{T}_{h}}^{2} \leq C_{a}^{2} h^{2 k+2}
$$

where $C_{a}$ is now independent of $h$ and $f_{h}$ and depends on $t$ and on the solution $(E, f)$ through its norm. This proves part (a) of the theorem. 
To prove part (b) of the theorem, we only need to modify slightly the estimates for $I_{2}$ and $\mathcal{K}^{2}$ which involve the approximation of the electrostatic field. The term $I_{2}$ is estimated similarly but using (32) from Lemma 5 (with $r=k+1$ ) to estimate the error $\left\|\mathbf{E}-\mathbf{E}_{h}\right\|_{0, \mathcal{T}_{h_{x}}^{x}}$. Thus,

$$
\left|I_{2}\right| \leq C h^{2 k+2}\|f\|_{1, \infty, \Omega}\left[\left\|\left|(\mathbf{E}(t), \Phi)\left\|\left.\right|_{k+2, \Omega_{\mathbf{x}}} ^{2}+C\right\| f \|_{k+1, \Omega}^{2}\right]+2 C\right\| f\left\|_{1, \infty, \Omega}\right\| \omega^{h} \|_{0, \mathcal{T}_{h}}^{2} .\right.
$$

To estimate $\mathcal{K}^{2}$, we only need to modify the estimate for $\left\|\mathbf{E}-\mathbf{E}_{h}\right\|_{0, \infty, \Omega_{\mathbf{x}}}$ used to bound $\mathcal{K}^{2}$ given in (58). Using now (33) from Lemma 6 (with $r=k+1$ ) together with estimate (22) from Lemma 2 and the approximation properties (12), we get

$$
\begin{aligned}
\left\|\mathbf{E}-\mathbf{E}_{h}\right\|_{0, \infty} \leq & C\left(\left\|\mathbf{E}-\mathcal{P}^{x}(\mathbf{E})\right\|_{0, \infty, \Omega_{\mathbf{x}}}+h^{-1}\left\|\Phi-\mathcal{P}^{x}(\Phi)\right\|_{0, \infty, \Omega_{\mathbf{x}}}\right) \\
& +C h^{3 / 2} \mid \log (h)\|\rho\|_{W^{3 / 2, d}\left(\Omega_{\mathbf{x}}\right)} \\
& +C h^{1-d / 2}|\log (h)|\left(C\left\|f-\mathcal{P}_{h}(f)\right\|_{0, \mathcal{T}_{h}}+\left\|f_{h}-\mathcal{P}_{h}(f)\right\|_{0, \mathcal{T}_{h}}\right),
\end{aligned}
$$

and so making use of (60) and the approximation properties (12), we get

$$
\begin{aligned}
\left\|\mathbf{E}-\mathbf{E}_{h}\right\|_{0, \infty, \mathcal{T}_{h_{x}}^{x}} \leq & C h\left(\|\mathbf{E}\|_{1, \infty, \Omega_{\mathbf{x}}}+\|\Phi\|_{2, \infty, \Omega_{\mathbf{x}}}\right)+C h^{3 / 2}|\log (h)|\|\rho\|_{W^{3 / 2, d}\left(\Omega_{\mathbf{x}}\right)} \\
& +C h^{1-d / 2}|\log (h)|\left(C h^{k+1}\|f\|_{k+1, \Omega}+\left\|\omega^{h}\right\|_{0, \mathcal{T}_{h}}\right),
\end{aligned}
$$

which except for the norm in the first term is the same bound we had for the $\mathrm{RT}_{k}$ approximation in case (a). Hence, the proof of part (b) can be completed proceeding exactly as before and therefore the details are omitted.

\section{EnERgy Conservation}

We now discuss how well the proposed schemes for approximating the VlasovPoisson system preserve the total energy. We show, following [6] that by appropriately tuning the coefficients of the LDG approximation of the Poisson problem, the total discrete energy is indeed conserved for the resulting LDG-DG method for the Vlasov-Poisson system. As a matter of fact, we can show such result under a technical restriction on the polynomial degree, namely $k \geq 2$.

We wish to observe that the resulting method requires the solution of $2 d$ (instead of one) $d$-dimensional Poisson problems. Although this might be considered as a drawback of the method, it should be noted that the solution of the Poisson problem is the low dimensional part (and so less computational expensive) of the whole computation.

This is given in next result.

Proposition 2 (Energy conservation). Let $r=k \geq 2$ and let $\left(\left(\mathbf{E}_{h}, \Phi\right), f_{h}\right) \in$ $\mathcal{C}^{1}\left([0, T] ;\left(\boldsymbol{\Xi}_{h}^{k} \times X_{h}^{k}\right) \times \mathcal{Z}_{h}^{k}\right)$ be the LDG(v)-DG approximation of the Vlasov-Poisson problem (1)-(3), solution of (19)-(30), with the numerical fluxes (17) for the 
approximate density. Let the numerical fluxes for the LDG approximation to (30) be given by:

$$
\left\{\begin{array}{l}
\left(\widehat{\mathbf{E}_{h}}\right)=\left\{\mathbf{E}_{h}\right\}+\frac{\operatorname{sign}(\mathbf{v} \cdot \mathbf{n})}{2} \llbracket \mathbf{E}_{h} \rrbracket \mathbf{n}-C_{11} \llbracket \Phi_{h} \rrbracket \\
\left(\widehat{\Phi_{h}}\right)=\left\{\Phi_{h}\right\}-\frac{\operatorname{sign}(\mathbf{v} \cdot \mathbf{n})}{2} \llbracket \Phi_{h} \rrbracket \cdot \mathbf{n},
\end{array}\right.
$$

where $C_{11}>0$ at all edges/faces. Then, the following identity holds true

$$
\frac{1}{2} \frac{d}{d t}\left(\sum_{R \in \mathcal{T}_{h}} \int_{R} f_{h}(t)|\mathbf{v}|^{2} d \mathbf{x} d \mathbf{v}+\left\|\mathbf{E}_{h}(t)\right\|_{0, \mathcal{T}_{h_{x}}^{x}}^{2}+\left\|C_{11}^{1 / 2} \llbracket \Phi_{h}(t) \rrbracket\right\|_{0, \mathcal{E}_{x}}^{2}\right)=0 .
$$

Proof. The proof is very similar to that given in [6, Section 5] for the case $d=1$. We however give it here for the sake of completeness.

\section{First step:}

In this first step, since $f \in \mathcal{Z}_{h}^{k}$ is a scalar function, we set $\boldsymbol{\tau}=\mathbf{v} f \in \boldsymbol{\Xi}_{h}^{k}$ in (27) and we integrate over all the elements of the partition $\mathcal{T}_{h_{v}}^{v}$

$$
\int_{\Omega_{\mathbf{v}}} \int_{T^{x}} \mathbf{E} \cdot \mathbf{v} f d \mathbf{x} d \mathbf{v}+\int_{\Omega_{\mathbf{v}}} \int_{T^{x}} \Phi \operatorname{div}_{\mathbf{x}}(\mathbf{v} f) d \mathbf{x} d \mathbf{v}-\int_{\Omega_{\mathbf{v}}} \int_{\partial T^{x}} \widehat{\Phi} f \mathbf{v} \cdot \mathbf{n} d s_{x} d \mathbf{v}=0
$$

and integrating by parts again and summing over all elements in $\mathcal{T}_{h_{x}}^{x}$, we get

$$
\begin{aligned}
\int_{\Omega} \mathbf{v} \cdot \nabla_{\mathbf{x}}^{h}(\Phi) f d \mathbf{x} d \mathbf{v}= & \sum_{R \in \mathcal{T}_{h}} \int_{R} \mathbf{E} \cdot \mathbf{v} f d \mathbf{x} d \mathbf{v} \\
& +\sum_{T^{x} \in \mathcal{T}_{h_{x}}^{x}} \int_{\Omega_{\mathbf{v}}} \int_{\partial T^{x}} \Phi f \mathbf{v} \cdot \mathbf{n} d s_{x} d \mathbf{v} \\
& -\sum_{T^{x} \in \mathcal{T}_{h_{x}}^{x}} \int_{\Omega_{\mathbf{v}}} \int_{\partial T^{x}} \widehat{\Phi} f \mathbf{v} \cdot \mathbf{n} d s_{x} d \mathbf{v} .
\end{aligned}
$$

Next, we set $\varphi_{h}=\Phi \in X_{h}^{k} \subset \mathcal{Z}_{h}^{k}$ in (19) ( $\Phi$ as a polynomial in $\mathcal{Z}_{h}^{k}$ is constant in $\mathbf{v}$ )

$$
\begin{aligned}
0= & \sum_{\mathcal{R} \in \mathcal{T}_{h}} \int_{\mathcal{R}} \frac{\partial f}{\partial t} \Phi d \mathbf{v} d \mathbf{x}-\int_{\Omega} f \mathbf{v} \cdot \nabla_{\mathbf{x}}^{h} \Phi d \mathbf{v} d \mathbf{x}+\int_{\Omega} f \mathbf{E} \cdot \nabla_{\mathbf{v}}^{h} \Phi d \mathbf{v} d \mathbf{x} \\
& +\sum_{T^{v} \in \mathcal{T}_{h_{v}^{v}}^{v}} \int_{T^{v}} \int_{\mathcal{E}_{x}}\{\mathbf{v} f\}_{\alpha} \cdot \llbracket \Phi \rrbracket d s_{x} d \mathbf{v}-\sum_{T^{x} \in \mathcal{T}_{h_{x}}^{x}} \int_{T^{x}} \int_{\mathcal{E}_{v}}\{\mathbf{E} f\}_{\beta} \cdot \llbracket \Phi \rrbracket d s_{v} d \mathbf{x} .
\end{aligned}
$$

Observe that the third and the last terms vanish; since $(\Phi)$ does not depend on $\mathbf{v}$, not only $\nabla_{\mathbf{v}}^{h} \Phi=0$ but also $\llbracket \rrbracket=0$ ( $\Phi$ is constant on $\left.\mathbf{v}\right)$, and the terms from the boundary of $\Omega_{\mathbf{v}}$ cancel due to the compact boundary conditions. Hence,

$$
0=\sum_{\mathcal{R} \in \mathcal{T}_{h}} \int_{\mathcal{R}} \frac{\partial f}{\partial t} \Phi d \mathbf{v} d \mathbf{x}-\int_{\Omega} f \mathbf{v} \cdot \nabla_{\mathbf{x}}^{h} \Phi d \mathbf{v} d \mathbf{x}+\sum_{T^{v} \in \mathcal{T}_{h_{v}^{v}}^{v}} \int_{T^{v}} \int_{\mathcal{E}_{x}}\{\mathbf{v} f\}_{\alpha} \cdot \llbracket \Phi \rrbracket d s_{x} d \mathbf{v} .
$$


Then, combing the result with (63) and using the periodic boundary conditions in $x$, we have

$$
\begin{aligned}
\sum_{\mathcal{R} \in \mathcal{T}_{h}} \int_{\mathcal{R}} \frac{\partial f}{\partial t} \Phi d \mathbf{v} d \mathbf{x}= & \sum_{R \in \mathcal{T}_{h}} \int_{R} \mathbf{E} \cdot \mathbf{v} f d \mathbf{x} d \mathbf{v}+\int_{T^{v}} \int_{\mathcal{E}_{x}^{0}}\{\Phi\} \llbracket \mathbf{v} f \rrbracket d s_{x} d \mathbf{v} \\
& +\sum_{T^{v} \in \mathcal{T}_{h_{v}}^{v}} \int_{T^{v}} \int_{\mathcal{E}_{x}}\left[\llbracket \Phi \rrbracket\{\mathbf{v} f\}-\{\mathbf{v} f\}_{\alpha} \cdot \llbracket \Phi \rrbracket-\widehat{\Phi} \llbracket \mathbf{v} f \rrbracket\right] d s_{x} d \mathbf{v}
\end{aligned}
$$

\section{Second step:}

Now, we differentiate with respect to time the first order system (23) and consider its DG approximation. The second equation (28) reads,

$$
\int_{T^{x}} \mathbf{E}_{t} \nabla_{\mathbf{x}} q d \mathbf{x}-\int_{\partial T^{x}} \widehat{\mathbf{E}_{t}} q \cdot \mathbf{n} d s_{x}=\int_{T^{x}} \rho_{t} q d \mathbf{x} \quad \forall q \in V_{h}^{r}
$$

where the definition for $\widehat{\mathbf{E}_{t}}$ corresponds to that chosen for $\widehat{\mathbf{E}}$ but with $(E, \Phi)$ replaced by $\left(\mathbf{E}_{t}, \Phi_{t}\right)$. By setting $p=\Phi$ in the above equation and replacing $\rho_{t}$ by its definition, we have

$$
\int_{T^{x}} \mathbf{E}_{t} \nabla_{\mathbf{x}} \Phi d \mathbf{x}-\int_{\partial T^{x}} \widehat{\mathbf{E}_{t}} \Phi \cdot \mathbf{n} d s_{x}=\sum_{T^{v} \in \mathcal{T}_{h_{v}}^{v}} \int_{T^{x}} \int_{T^{v}} f_{t} \Phi d \mathbf{v} d \mathbf{x}
$$

Now, taking $z=\mathbf{E}_{t}$ in (27) and integrating by parts the volume term on the right hand side of that equation, we find

$$
\int_{T^{x}} \mathbf{E} \cdot \mathbf{E}_{t} d \mathbf{x}-\int_{T^{x}} \nabla_{\mathbf{x}}(\Phi) \mathbf{E}_{t} d \mathbf{x}+\int_{\partial T^{x}} \Phi \mathbf{E}_{t} \cdot \mathbf{n} d s_{\mathbf{x}}-\int_{\partial T^{x}} \widehat{\Phi} \mathbf{E}_{t} \cdot \mathbf{n} d s_{x}=0 .
$$

Then, combining (65) with the above equation and summing over all elements of $\mathcal{T}_{h_{x}}^{x}$ and using (9) together with the periodicity of the boundary conditions for the Poisson problem, we get

$$
\begin{aligned}
\int_{\Omega_{\mathbf{x}}} \mathbf{E} \cdot \mathbf{E}_{t} d \mathbf{x}= & \int_{\Omega} f_{t} \Phi d \mathbf{v} d \mathbf{x} \\
& +\int_{\mathcal{E}_{x}}\left(\widehat{\mathbf{E}_{t}} \llbracket \Phi \rrbracket+\widehat{\Phi} \llbracket \mathbf{E}_{t} \rrbracket-\llbracket \Phi \rrbracket\left\{\mathbf{E}_{t}\right\}\right) d s_{x}-\int_{\mathcal{E}_{x}^{o}}\{\Phi\} \llbracket \mathbf{E}_{t} \rrbracket d s_{x}
\end{aligned}
$$




\section{Third step:}

We now proceed as in the proof for the continuous case and we take $\varphi=\frac{|\mathbf{v}|^{2}}{2}$ in $(19)$,

$$
\begin{aligned}
0 & =\sum_{\mathcal{R} \in \mathcal{T}_{h}} \int_{\mathcal{R}} \frac{\partial f}{\partial t} \frac{|\mathbf{v}|^{2}}{2} d \mathbf{v} d \mathbf{x}-\int_{\Omega} f \mathbf{v} \cdot \nabla_{\mathbf{x}}^{h}\left(\frac{|\mathbf{v}|^{2}}{2}\right) d \mathbf{v} d \mathbf{x}+\int_{\Omega} f \mathbf{E} \cdot \nabla_{\mathbf{v}}^{h}\left(\frac{|\mathbf{v}|^{2}}{2}\right) d \mathbf{v} d \mathbf{x} \\
& +\frac{1}{2} \sum_{T^{v} \in \mathcal{T}_{h_{v}}^{v}} \int_{T^{v}} \int_{\mathcal{E}_{x}}\{\mathbf{v} f\}_{\alpha} \cdot \llbracket|\mathbf{v}|^{2} \rrbracket d s_{x} d \mathbf{v}-\frac{1}{2} \sum_{T^{v} \in \mathcal{T}_{h_{v}}^{v}} \int_{T^{x}} \int_{\mathcal{E}_{v}}\left\{\mathbf{E}_{h} f\right\}_{\beta} \cdot \llbracket \frac{|\mathbf{v}|^{2}}{2} \rrbracket d s_{v} d \mathbf{x} .
\end{aligned}
$$

The second and fourth terms vanish since $\mathbf{v}$ is independent of $\mathbf{x}$ and last term. Then, using the consistency of the numerical fluxes $(\widehat{\mathbf{v} \cdot \mathbf{n} f})$ and $\widehat{\mathbf{E} \cdot \mathbf{n} f}$ (see (18)), the boundary terms telescope and no boundary term is left due to the periodic and compact boundary conditions. Hence, we simply get

$$
0=\sum_{\mathcal{R} \in \mathcal{T}_{h}} \int_{\mathcal{R}} \frac{\partial f}{\partial t} \frac{|\mathbf{v}|^{2}}{2} d \mathbf{v} d \mathbf{x}+\int_{\Omega} \mathbf{E} \cdot \mathbf{v} f d \mathbf{v} d \mathbf{x} .
$$

Next, we use equation (64) to substitute the last term in (67),

$$
\begin{aligned}
0= & \sum_{\mathcal{R} \in \mathcal{T}_{h}} \int_{\mathcal{R}} \frac{\partial f}{\partial t} \frac{|\mathbf{v}|^{2}}{2} d \mathbf{v} d \mathbf{x}+\sum_{\mathcal{R} \in \mathcal{T}_{h}} \int_{\mathcal{R}} \frac{\partial f}{\partial t} \Phi d \mathbf{v} d \mathbf{x}-\sum_{T^{v} \in \mathcal{T}_{h_{v}}^{v}} \int_{T^{v}} \int_{\mathcal{E}_{x}^{o}}\{\Phi\} \llbracket \mathbf{v} f \rrbracket d s_{x} d \mathbf{v} \\
& +\sum_{T^{v} \in \mathcal{T}_{h_{v}}^{v}} \int_{T^{v}} \int_{\mathcal{E}_{x}}\left[\{\mathbf{v} f\}_{\alpha} \cdot \llbracket \Phi \rrbracket+\widehat{\Phi} \llbracket \mathbf{v} f \rrbracket-\llbracket \Phi \rrbracket\{\mathbf{v} f\}\right] d s_{x} d \mathbf{v} .
\end{aligned}
$$

Finally, we substitute the second volume term above by means of (66),

$$
\begin{aligned}
& 0=\sum_{\mathcal{R} \in \mathcal{T}_{h}} \int_{\mathcal{R}} \frac{\partial f}{\partial t} \frac{|\mathbf{v}|^{2}}{2} d \mathbf{v} d \mathbf{x}+\int_{\Omega_{\mathbf{x}}} \mathbf{E} \cdot \mathbf{E}_{t} d \mathbf{x}-\sum_{T^{v} \in \mathcal{T}_{h_{v}}^{v}} \int_{T^{v}} \int_{\mathcal{E}_{x}^{o}} \llbracket \Phi \rrbracket\{\mathbf{v} f\} d s_{x} d \mathbf{v} \\
& +\sum_{T^{v} \in \mathcal{T}_{h_{v}}^{v}} \int_{T^{v}} \int_{\mathcal{E}_{x}}\left[\{\mathbf{v} f\}_{\alpha} \cdot \llbracket \Phi \rrbracket+\widehat{\Phi} \llbracket \mathbf{v} f \rrbracket-\{\Phi\} \llbracket \mathbf{v} f \rrbracket\right] d s_{x} d \mathbf{v} \\
& +\int_{\mathcal{E}_{x}}\left(\llbracket \Phi \rrbracket\left\{\mathbf{E}_{t}\right\}-\widehat{\mathbf{E}_{t}} \llbracket \Phi \rrbracket-\widehat{\Phi} \llbracket \mathbf{E}_{t} \rrbracket\right) d s_{x}+\int_{\mathcal{E}_{x}^{o}}\{\Phi\} \llbracket \mathbf{E}_{t} \rrbracket d s_{x} .
\end{aligned}
$$

Then, for each $e \in \mathcal{E}_{x}$, we define

$$
\begin{aligned}
& \Theta_{e}^{H}= \begin{cases}\llbracket \Phi \rrbracket\left\{\mathbf{E}_{t}\right\}-\widehat{\mathbf{E}_{t}} \llbracket \Phi \rrbracket-\widehat{\Phi} \llbracket \mathbf{E}_{t} \rrbracket+\{\Phi\} \llbracket \mathbf{E}_{t} \rrbracket & \text { on } e \in \mathcal{E}_{x}^{o} \\
\llbracket \Phi \rrbracket\left\{\mathbf{E}_{t}\right\}-\widehat{\mathbf{E}_{t}} \llbracket \Phi \rrbracket-\widehat{\Phi} \llbracket \mathbf{E}_{t} \rrbracket & \text { on } e \in \mathcal{E}_{x}^{\partial}\end{cases} \\
& \Theta_{e}^{F}= \begin{cases}\{\mathbf{v} f\}_{\alpha} \cdot \llbracket \Phi \rrbracket+\widehat{\Phi} \llbracket \mathbf{v} f \rrbracket-\{\Phi\} \llbracket \mathbf{v} f \rrbracket-\llbracket \Phi \rrbracket\{\mathbf{v} f\} & \text { on } e \in \mathcal{E}_{x}^{o} \\
\{\mathbf{v} f\}_{\alpha} \cdot \llbracket \Phi \rrbracket+\widehat{\Phi} \llbracket \mathbf{v} f \rrbracket-\llbracket \Phi \rrbracket\{\mathbf{v} f\} & \text { on } e \in \mathcal{E}_{x}^{\partial}\end{cases}
\end{aligned}
$$


so that (68) can be rewritten as

$$
\begin{aligned}
\frac{1}{2} \frac{\partial}{\partial t}\left(\sum_{\mathcal{R} \in \mathcal{T}_{h}} \int_{\mathcal{R}} f|\mathbf{v}|^{2} d \mathbf{v} d \mathbf{x}+\int_{\Omega_{\mathbf{x}}}|\mathbf{E}|^{2} d \mathbf{x}\right) & +\sum_{T^{v} \in \mathcal{T}_{h_{v}}^{v}} \sum_{e \in \mathcal{E}_{x}} \int_{T^{v}} \int_{e} \Theta_{e}^{F} d s_{\mathbf{x}} d \mathbf{v} \\
& +\sum_{e \in \mathcal{E}_{x}} \int_{e} \Theta_{e}^{H} d s_{\mathbf{x}}=0
\end{aligned}
$$

Thus, we only need to show that $\Theta_{e}^{H}$ and $\Theta_{e}^{F}$ are either zero or the time derivative of a non-negative function for all $e \in \mathcal{E}_{x}$.

Next, for $e \in \mathcal{E}_{x}^{0}$, using the definition of the numerical fluxes (61) for the LDG approximation together with the fact that $(\mathbf{E}, \Phi)$ is $\mathcal{C}^{1}$ in time, we find

$$
\begin{aligned}
& \Theta_{e}^{H}=\llbracket \Phi \rrbracket\left\{\mathbf{E}_{t}\right\}-\widehat{\mathbf{E}_{t}} \llbracket \Phi \rrbracket-\widehat{\Phi} \llbracket \mathbf{E}_{t} \rrbracket+\{\Phi\} \llbracket \mathbf{E}_{t} \rrbracket=c_{11} \llbracket \Phi_{t} \rrbracket \cdot \llbracket \Phi \rrbracket=\frac{1}{2} \frac{\partial}{\partial t}\left(c_{11}|\llbracket \Phi \rrbracket|^{2}\right) \\
& \forall e \in \mathcal{E}_{x}^{0} \text {. }
\end{aligned}
$$

Similarly, for $e \in \mathcal{E}_{x}^{\partial}$ taking into account the definition at boundary edges/faces, we have $\Theta_{e}^{H}=c_{11} \llbracket \Phi_{t} \rrbracket \cdot \llbracket \Phi \rrbracket$ on $e \in \mathcal{E}_{x}^{\partial}$. Hence, arguing as before and putting together the result with the above identity we arrive at

$$
\Theta_{e}^{H}=c_{11} \llbracket \Phi_{t} \rrbracket \cdot \llbracket \Phi \rrbracket=\frac{1}{2} \frac{\partial}{\partial t}\left(c_{11}|\llbracket \Phi \rrbracket|^{2}\right) \quad \forall e \in \mathcal{E}_{x} .
$$

We next deal with $\Theta_{e}^{F}$. Notice that for $e \in \mathcal{E}_{x}^{\partial}$ it is easy to see, using the definition of the numerical fluxes $\widehat{\mathbf{v f f}}$ and $\widehat{\Phi}$ at $\partial \Omega_{\mathbf{x}}$, that $\Theta_{e}^{F} \equiv 0$ for all $e \in \mathcal{E}_{x}^{\partial}$.

Now, for $e \in \mathcal{E}_{x}^{o}$, from the definition of the numerical fluxes $\widehat{\mathbf{v f f}}$ and $\widehat{\Phi}$ given in (18) and (61), respectively we find for $\Theta_{e}^{F}$,

$$
\begin{array}{rlrl}
\Theta_{e}^{F} & =\{\mathbf{v} f\}_{\alpha} \cdot \llbracket \Phi \rrbracket+\widehat{\Phi} \llbracket \mathbf{v} f \rrbracket-\{\Phi\} \llbracket \mathbf{v} f \rrbracket-\llbracket \Phi \rrbracket \cdot\{\mathbf{v} f\} & \\
& =\frac{|\mathbf{v} \cdot \mathbf{n}|}{2} \llbracket f \rrbracket \cdot \llbracket \Phi \rrbracket-\mathbf{C}_{\mathbf{1 2}} \cdot \llbracket \Phi \rrbracket \llbracket \mathbf{v} f \rrbracket \\
& =\frac{|\mathbf{v} \cdot \mathbf{n}|}{2} \llbracket f \rrbracket \cdot \llbracket \Phi \rrbracket-\frac{\operatorname{sign}(\mathbf{v} \cdot \mathbf{n})}{2} \mathbf{n} \cdot \llbracket \Phi \rrbracket \mathbf{v} \cdot \llbracket f \rrbracket & \\
& =\frac{1}{2} \llbracket f \rrbracket \cdot \llbracket \Phi \rrbracket(|\mathbf{v} \cdot \mathbf{n}|-|\mathbf{v} \cdot \mathbf{n}|)=0 & e \in \mathcal{E}_{x}^{0}
\end{array}
$$

and so substituting the above result together with (70) into (69) we reach (62).

For other DG-DG schemes, inequalities similar to those given in $[7,6]$ can be proved. 


\section{Appendix A. Error Analysis for the approximation of the ELECTROSTATIC FIELD}

This appendix is devoted to show the results stated in $\S 3$ related to the approximation of the Electrostatic field. We start by showing the auxiliary result, Lemma 2, which bounds the error in the density in terms of the error in the distribution function. Then we prove the energy norm estimates for the $\mathrm{RT}_{k}$ and LDG approximation, given in Lemmas 3 and 5 , respectively. The $L^{\infty}$-bounds for both methods are given at the end of the appendix.

A.1. Proof of Lemma 2. The proof of estimate (21) follows straightforwardly from the definitions (2) and (20) of $\rho$ and $\rho_{h}$, respectively, and Hölder inequality.

To show (22), we first prove that

$$
\left\|\rho-\rho_{h}\right\|_{-1, \infty, \mathcal{T}_{h_{x}}^{x}} \leq C\left\|\rho-\rho_{h}\right\|_{L^{d}\left(\mathcal{T}_{h_{x}}^{x}\right)} .
$$

Note that from the mass conservation given in (4) and Lemma 1 for $\rho$ and $\rho_{h}$, respectively, it follows that $\left[\rho-\rho_{h}\right]$ is orthogonal to the global constants. Hence, denoting by $<q>_{\Omega_{\mathbf{x}}}=\left(1 /\left|\Omega_{\mathbf{x}}\right|\right) \int_{\Omega_{\mathbf{x}}} q d \mathbf{x}$ the average of a function $q$, Hölder inequality together with Poincare-Friederich's inequality [17, Theorem 4.1] (which shows the Sobolev's imbedding $W^{1,1}\left(\Omega_{\mathbf{x}}\right) \subset L^{q^{*}}\left(\Omega_{\mathbf{x}}\right)$ with $q^{*}=d /(d-1)$ for DG functions, see also [2, Lemma 5.10] for the continuous counterpart) gives,

$$
\begin{aligned}
\left\|\rho-\rho_{h}\right\|_{-1, \infty, \mathcal{T}_{h x}^{x}} & =\sup _{q \in W_{h}^{1,1}\left(\mathcal{T}_{h_{x}}^{x}\right)} \frac{\left|\int_{\Omega_{\mathbf{x}}}\left(\rho-\rho_{h}\right) q d \mathbf{x}\right|}{\|q\|_{W_{h}^{1,1}\left(\mathcal{T}_{h_{x}}^{x}\right)}} \\
& =\sup _{q \in W_{h}^{1,1}\left(\mathcal{T}_{h_{x}}^{x}\right)} \frac{\left|\int_{\Omega_{\mathbf{x}}}\left(\rho-\rho_{h}\right)[q-\langle q\rangle] d \mathbf{x}\right|}{\|q\|_{W_{h}^{1,1}\left(\mathcal{T}_{h_{x}}^{x}\right)}} \\
& \leq \sup _{q \in W_{h}^{1,1}\left(\mathcal{T}_{h_{x}}^{x}\right)} \frac{\left\|\rho-\rho_{h}\right\|_{L^{d}\left(\mathcal{T}_{h_{x}}^{x}\right)}\|q-\langle q\rangle\|_{L^{d / d-1}\left(\mathcal{T}_{h_{x}}^{x}\right)}}{\|q\|_{W_{h}^{1,1}\left(\mathcal{T}_{h_{x}}^{x}\right)}} \leq C\left\|\rho-\rho_{h}\right\|_{L^{d}\left(\mathcal{T}_{h_{x}}^{x}\right)} .
\end{aligned}
$$

To conclude we only need to bound the error in the $L^{d}$-norm. Triangle inequality together with the $L^{d}$-stability of the $L^{2}$-projection (11) and inverse inequality, gives

$$
\begin{aligned}
\left\|\rho-\rho_{h}\right\|_{L^{d}\left(\mathcal{T}_{h x}^{x}\right)} & \leq\left\|\rho-\mathcal{P}_{\mathbf{x}}(\rho)\right\|_{L^{d}\left(\mathcal{T}_{h_{x}}^{x}\right)}+\left\|\mathcal{P}_{\mathbf{x}}(\rho)-\rho_{h}\right\|_{L^{d}\left(\mathcal{T}_{h_{x}}^{x}\right)} \\
& \leq C h^{3 / 2}|\rho|_{W^{3 / 2, d}\left(\Omega_{\mathbf{x}}\right)}+C h_{x}^{-d\left(\frac{1}{2}-\frac{1}{d}\right)}\left\|\mathcal{P}_{\mathbf{x}}(\rho)-\rho_{h}\right\|_{0, \mathcal{T}_{h_{x}}^{x}} .
\end{aligned}
$$


Next, taking into account the definition of the continuous and discrete density, using that the projection $\mathcal{P}^{\mathbf{x}}$ is independent of $\mathbf{v}$ and Hölder inequality, we find

$$
\begin{aligned}
\left\|\mathcal{P}^{\mathbf{x}}(\rho)-\rho_{h}\right\|_{0, \mathcal{T}_{h x}^{x}} & =\sum_{T^{x} \in \mathcal{T}_{h_{x}}^{x}} \int_{T^{x}}\left|\sum_{T^{v} \in \mathcal{T}_{h_{v}^{v}}^{v}} \int_{T^{v}}\left[\mathcal{P}_{x}(f)-f_{h}\right] d \mathbf{v}\right| d \mathbf{x} \\
& \leq C\left[\operatorname{meas}\left(\Omega_{\mathbf{v}}\right)\right]^{1 / 2}\left\|\mathcal{P}_{x}(f)-f_{h}\right\|_{0, \mathcal{T}_{h}} \\
& \leq C L^{d / 2}\left(\left\|\mathcal{P}_{x}(f)-\mathcal{P}_{h}(f)\right\|_{0, \mathcal{T}_{h}}+\left\|\mathcal{P}_{h}(f)-f_{h}\right\|_{0, \mathcal{T}_{h}}\right),
\end{aligned}
$$

where in the last step we have added and susbtracted $\mathcal{P}_{h}(f)$ and used triangle inequality. Now, using the $L^{2}$-stability of the $\mathcal{P}_{\mathbf{x}}$-projection together with the approximation property (13) we have for the first term above

$$
\begin{aligned}
\left\|\mathcal{P}_{x}(f)-\mathcal{P}_{h}(f)\right\|_{0, \mathcal{T}_{h}} & =\left\|\left[\mathcal{P}_{x} \otimes \mathbb{I}_{\mathbf{v}}\right](f)-\left[\mathcal{P}_{\mathbf{x}} \otimes \mathcal{P}_{\mathbf{v}}\right](f)\right\|_{0, \mathcal{T}_{h}}=\left\|\mathcal{P}_{x}\left[f-\mathcal{P}_{\mathbf{v}}(f)\right]\right\|_{0, \mathcal{T}_{h}} \\
& \leq C\left\|f-\mathcal{P}_{\mathbf{v}}(f)\right\|_{0, \mathcal{T}_{h}} \leq C h_{v}^{k+1}\|f\|_{k+1, \mathcal{T}_{h}} .
\end{aligned}
$$

Substituting this estimate in (73) and the result in (72) we get (71), which implies (22) and the proof is complete.

A.2. Proof of Lemma 3. To simplify the notation we drop the dependence on the $t$ variable. From [15, II. Proposition 2.16] it follows that

$$
\left\|\mathbf{E}-\mathbf{E}_{h}\right\|_{H\left(\operatorname{div} ; \Omega_{\mathbf{x}}\right)}+\left\|\Phi-\Phi_{h}\right\|_{0, \Omega_{\mathbf{x}}} \leq C\left(\inf _{\boldsymbol{\tau} \in \boldsymbol{\Sigma}_{h}^{k}}\|\mathbf{E}-\boldsymbol{\tau}\|_{H\left(\operatorname{div} ; \Omega_{\mathbf{x}}\right)}+\inf _{q \in Q_{h}^{k}}\|\Phi-q\|_{0, \mathcal{T}_{h_{x}}^{x}}+M_{3 h}\right),
$$

where $M_{3 h}$ is the consistency error:

$$
M_{3 h}:=\sup _{q \in Q_{h}^{k}} \frac{\left|\int_{\Omega_{\mathbf{x}}}\left(\rho-\rho_{h}\right) q d \mathbf{x}\right|}{\|q\|_{0, \mathcal{T}_{h_{x}}^{x}}} .
$$

The first two terms in (74) are readily estimated from the standard approximation properties of Raviart-Thomas elements; estimates (15) and the approximation of the $L_{0}^{2}$-projection (10),

$$
\inf _{\boldsymbol{\tau} \in \boldsymbol{\Sigma}_{h}^{k}}\|\mathbf{E}-\boldsymbol{\tau}\|_{H\left(\operatorname{div} ; \Omega_{\mathbf{x}}\right)}+\inf _{q \in Q_{h}^{k}}\|\Phi-q\|_{0, \mathcal{T}_{h_{x}}^{x}} \leq C h^{k+1}\left(\|\mathbf{E}(t)\|_{k+1, \Omega_{\mathbf{x}}}+\|\Phi\|_{k+2, \Omega_{\mathbf{x}}}\right) .
$$

Using Cauchy-Schwarz inequality, together with estimate (21) from Lemma 2 we find

$$
M_{3 h} \leq C\left\|\rho-\rho_{h}\right\|_{0, \mathcal{T}_{h_{x}}^{x}} \leq C L^{d / 2}\left\|f-f_{h}\right\|_{0, \mathcal{T}_{h}},
$$

and the proof of the estimate in the $H\left(\operatorname{div} ; \Omega_{\mathbf{x}}\right)$-norm is complete. 
A.3. Proof of Lemma 5. We start by noticing that if we denote by $(\widetilde{\mathbf{E}}, \widetilde{\Phi})$ the solution of the discrete Poisson problem (23), triangle inequality gives

$$
\left|\left(\mathbf{E}-\mathbf{E}_{h}, \Phi-\Phi_{h}\right)\right|_{\mathcal{A}} \leq|(\mathbf{E}-\widetilde{\mathbf{E}}, \Phi-\widetilde{\Phi})|_{\mathcal{A}}+\left|\left(\widetilde{\mathbf{E}}-\mathbf{E}_{h}, \widetilde{\Phi}-\Phi_{h}\right)\right|_{\mathcal{A}}
$$

The last term above is estimated proceeding exactly as in [18] (where the dirichlet problem is treated) and the same error estimate can be shown (for the case of interest, $C_{11}$ of order $O(1 / h)$ and $C_{22}$ either zero or of order $\left.O(1)\right)$ :

$$
\left|\left(\widetilde{\mathbf{E}}-\mathbf{E}_{h}, \widetilde{\Phi}-\Phi_{h}\right)\right|_{\mathcal{A}} \leq C h^{r}\left\||(\widetilde{\mathbf{E}}, \widetilde{\Phi}) \||_{r+1} .\right.
$$

We omit the details for the sake of conciseness. The first term in (75) is estimated by using standard regularity theorems for the Poisson problem [29] together with the Poincare-Friederichs inequality for discrete functions in $Q_{h}^{r}$ (see [11]) and estimate (21) from Lemma 2

$$
\begin{aligned}
|(\mathbf{E}-\widetilde{\mathbf{E}}, \Phi-\widetilde{\Phi})|_{\mathcal{A}} & =\|\mathbf{E}-\widetilde{\mathbf{E}}\|_{0, \mathcal{T}_{h_{x}}^{x}} \leq C\left\|\rho-\rho_{h}\right\|_{-1, \mathcal{T}_{h_{x}}^{x}}=\sup _{q_{h} \in Q_{h}^{r}} \frac{\int_{\mathcal{T}_{h_{x}}^{x}}\left(\rho-\rho_{h}\right) q_{h} d \mathbf{x}}{\left\|q_{h}\right\|_{1, \mathcal{T}_{h}}} \\
& \leq C C_{p}\left\|\rho-\rho_{h}\right\|_{0, \mathcal{T}_{h_{x}}^{x}} \leq C L^{d / 2}\left\|f-f_{h}\right\|_{0, \mathcal{T}_{h}} .
\end{aligned}
$$

Hence, substituting this estimate together with (76) into (75) concludes the proof.

A.4. $\boldsymbol{L}^{\infty}$-error estimates for the approximation to the electrostatic field. We next show the error estimates in the $L^{\infty}$-norm for the approximate electrostatic field with $R T_{k}$ and the LDG methods. For both methods, there are already available in the literature, $L^{\infty}$ and pointwise error analysis for the approximation of a linear Poisson problem (see [28] and [19]). Here, we will mainly modify the proof of those results in order to account for the nonlinearity of the Poisson problem (3). For that purpose, we argue similarly as in [38, 37], where the authors prove $L^{\infty}$ error estimates for the conforming approximation of a "general" Poisson problem taking into account the outside influence of the forcing term in the Poisson problem. However since [38, 37] deals with standard conforming approximation, some of the results and arguments used by the authors in these works cannot be straightforwardly adapted, in particular for the LDG approximation. We wish to stress that we are not concerned here with providing pointwise and localized error estimates. Our main goal is to show Lemmas 4 and 6 which in turn give the $L^{\infty}$-error estimates required by our analysis.

We next recall a result that will be used in the proof of both lemmas.

Let $\boldsymbol{\varphi} \in H\left(\operatorname{div}, \Omega_{\mathbf{x}}\right)$ be such that $\nabla \cdot \varphi \in L_{0}^{2}(\Omega)$. Let $g \in H^{1}\left(\Omega_{\mathbf{x}}\right) \cap L_{0}^{2}\left(\Omega_{\mathbf{x}}\right)$ be the solution of the problem

$$
-\Delta g=\nabla \cdot \varphi \quad \text { in } \Omega_{\mathbf{x}}
$$


with $g$ and $\nabla g$ subject to periodic boundary conditions on $\partial \Omega_{\mathbf{x}}$ We shall need the following a priori estimates in $L^{p}(\Omega)$-based norms for problem (77),

$$
\|g\|_{W^{1, p}\left(\Omega_{\mathbf{x}}\right)} \leq \frac{C}{p-1}\|\nabla \cdot \varphi\|_{W^{-1, p}\left(\Omega_{\mathbf{x}}\right)} \leq \frac{C}{p-1}\|\varphi\|_{L^{p}\left(\Omega_{\mathbf{x}}\right)}, \quad 1<p \leq 2 .
$$

The above estimate can be shown from the a-priori $L^{p}$-estimates for problem (77) (see for instance [29]) but tracing the constants through the proof of those results to get a precise dependence on $p$ of the leading constant in estimate (78). We also mention that for general polyhedral domains and dirichlet or neuman b.c., the range of $p$ is more restricted (see [25, 24] and also [23] for related work).

A.4.1. $\boldsymbol{L}^{\infty}$-error estimates for the $R T_{\boldsymbol{k}}$ approximation to the electrostatic field. We now show Lemma 4. We wish to stress, that the proof of this lemma follows essentially from [28, Lemma 4.1]. In [28], the authors give a general abstract framework which provides optimal $L^{\infty}\left(\Omega_{\mathbf{x}}\right)$ - error estimates for the mixed finite element approximation of a linear Poisson problem with Dirichlet boundary conditions. They use Nitsche's method of weighted Sobolev-norms [36] (see also [34] and [35]), in which the key idea is that by using weighted norms one can still work in $L^{2}$ rather than in $L^{\infty}$ and in particular, can still use duality arguments. In fact, the essential ingredient in their analysis is a duality argument combined with an a-priori estimate in certain weighted norm. Their result is rather general, since it covers Raviart-Thomas-Nedelec mixed methods and also Brezzi-DouglasMarini and Brezzi-Douglas-Fortin-Marini mixed approximations. Moreover, it is valid for any space dimension $d \geq 2$, and holds for partitions made of simplices or rectangulars.

In our case, in order to account for the nonlinearity on the right hand side of the Poisson problem, we only need to modify a single step in the proof of $[28$, Lemma 4.1] Hence, here we will only sketch the differences and the step that needs to be modified and we refer the reader to [28] for further details. We wish to stress that although the authors deal with the Dirichlet problem, all the error estimates proved in [28] carry over for the periodic Poisson problem.

We recall now some notation that will be required for using their results. The weight function $\sigma$ is defined by

$$
\sigma(x):=\left(\left|\mathbf{x}-\mathbf{x}_{0}\right|^{2}+\theta^{2}\right)^{1 / 2}, \quad \mathbf{x}, \mathbf{x}_{0} \in \Omega_{\mathbf{x}}
$$

where $|\cdot|$ denotes here the Euclidean distance in $\mathbb{R}^{d}$ and $\theta=C^{*} h$ with a constant $C^{*} \geq 1$ to be specified later on. The weight satisfies the non-oscillation property [36]:

$$
\max _{\mathbf{x} \in T^{x}} \sigma(\mathbf{x}) \leq C \min _{\mathbf{x} \in T^{x}} \sigma(\mathbf{x}), \quad \forall T^{x} \in \mathcal{T}_{h_{x}}^{x} .
$$

For $\alpha \in \mathbb{R}$, we defined the following weighted Sobolev norm:

$$
\|u\|_{\sigma^{\alpha}}^{2}:=\int_{\Omega_{\mathbf{x}}} \sigma^{\alpha}|u|^{2} d \mathbf{x}, \quad \forall u \in L^{2}\left(\Omega_{\mathbf{x}}\right), \quad \alpha \in \mathbb{R} .
$$


The following relations can be established between the weighted and $L^{\infty}$-norms

$$
\begin{gathered}
\|u\|_{\sigma^{-\alpha}} \leq C\|u\|_{0, \infty, \Omega_{\mathbf{x}}}\left\{\begin{array}{ll}
\theta^{(d-\alpha) / 2} & \alpha>d, \\
|\log \theta|^{1 / 2} & \alpha=d,
\end{array} \quad u \in L^{\infty}\left(\Omega_{\mathbf{x}}\right)\right. \\
\|\chi\|_{0, \infty, \Omega_{\mathbf{x}}} \leq C\left(\theta^{\alpha} / h^{d}\right)^{1 / 2}\|\chi\|_{\sigma^{-\alpha}} \quad \alpha \in \mathbb{R} \quad \chi \in \Sigma_{h}^{k} \text { or } \chi \in Q_{h}^{k} .
\end{gathered}
$$

We have now all ingredients to show Lemma 4:

Proof of Lemma 4 Let $\mathcal{R}_{h}^{k}: H\left(\operatorname{div} ; \Omega_{\mathbf{x}}\right) \longrightarrow \Sigma_{h}^{k}$ be the Raviart-Thomas projection as defined $\S 2$. Triangle inequality gives,

$$
\left\|\mathbf{E}-\mathbf{E}_{h}\right\|_{0, \infty, \Omega_{\mathbf{x}}} \leq\left\|\mathbf{E}-\mathcal{R}_{h}^{k}(\mathbf{E})\right\|_{0, \infty, \Omega_{\mathbf{x}}}+\left\|\mathcal{R}_{h}^{k}(\mathbf{E})-\mathbf{E}_{h}\right\|_{0, \infty, \Omega_{\mathbf{x}}} .
$$

Hence, we only need to estimate the last term above on right hand side. We shall show

$$
\left\|\mathcal{R}_{h}^{k}(\mathbf{E})-\mathbf{E}_{h}\right\|_{0, \infty, \Omega_{\mathbf{x}}} \leq C\left\|\mathbf{E}-\mathcal{R}_{h}^{k}(\mathbf{E})\right\|_{0, \infty, \Omega_{\mathbf{x}}}+C|\log (h)|\left\|\rho-\rho_{h}\right\|_{-1, \infty, \mathcal{T}_{h_{x}}^{x}},
$$

and so, substituting this estimate above and using standard approximation properties, the proof of the Lemma will be complete. Thus, it is enough to prove (83).

To show (83) arguing as in [28, Lemma 4.1], it turns out we only need to modify one step in the proof of [28, Lemma 4.1]; the bound for the $V$-term. In such step the authors were using the Galerkin orthogonality property of $\operatorname{div}\left(\mathbf{E}-\mathbf{E}_{h}\right)$ being orthogonal to $Q_{h}^{k}$, which due to the nonlinearity in Poisson is obviously not true in the present case. Since $\mathcal{R}_{h}^{k}(\mathbf{E})-\mathbf{E}_{h} \in \Sigma_{h}^{k}$, from (82) one has:

$$
\left\|\mathcal{R}_{h}^{k}(\mathbf{E})-\mathbf{E}_{h}\right\|_{0, \infty, \Omega_{\mathbf{x}}} \leq C\left(\theta^{(d+\alpha)} / h^{d}\right)^{1 / 2}\left\|\mathcal{R}_{h}^{k}(\mathbf{E})-\mathbf{E}_{h}\right\|_{\sigma^{-(d+\alpha)}} \quad 0<\alpha<2 .
$$

Following [28, Lemma 4.1] it can be shown that

$\left\|\mathcal{R}_{h}^{k}(\mathbf{E})-\mathbf{E}_{h}\right\|_{\sigma^{-(d+\alpha)}}^{2} \leq C(h / \theta)\left\|\mathcal{R}_{h}^{k}(\mathbf{E})-\mathbf{E}_{h}\right\|_{\sigma^{-(d+\alpha)}}^{2}+C\left\|\mathcal{R}_{h}^{k}(\mathbf{E})-\mathbf{E}\right\|_{\sigma^{-(d+\alpha)}}^{2}+|V|$, where the $V$-term reads (after integration by parts)

$$
V=-\int_{\Omega_{\mathbf{x}}}\left(\mathbf{E}-\mathbf{E}_{h}\right) \nabla u d \mathbf{x}=\int_{\Omega_{\mathbf{x}}} \operatorname{div}\left(\mathbf{E}-\mathbf{E}_{h}\right) u d \mathbf{x},
$$

where $u$ is the solution of the dual problem:

$$
\text { Find } u \in H^{1}\left(\Omega_{\mathbf{x}}\right) \cap L_{0}^{2}\left(\Omega_{\mathbf{x}}\right):-\Delta u=\operatorname{div} \mathcal{R}_{h}^{k}(\boldsymbol{\psi}),
$$

with periodic boundary conditions (for $u$ and for $\nabla u$ ). In the above dual problem, $\boldsymbol{\psi}$ is defined as

$$
\boldsymbol{\psi}=\sigma^{-(\alpha+n)}\left(\mathcal{R}_{h}^{0}(\mathbf{E})-\mathbf{E}_{h}\right) .
$$

Notice that in view of (14) the above problem is well posed. To estimate the term in (86), we first observe that $\operatorname{div} \mathbf{E}_{h} \in Q_{h}^{k}$ and $\operatorname{div}\left(\mathbf{E}-\mathbf{E}_{h}\right)=\left[1-\rho-\left(1-\rho_{h}\right)\right]=$ 
$\left[\rho_{h}-\rho\right]$. Hence, we can rewrite the term $V$ as

$$
V=\int_{\Omega_{\mathbf{x}}} \operatorname{div}\left(\mathbf{E}-\mathbf{E}_{h}\right) u d \mathbf{x}=\int_{\Omega_{\mathbf{x}}}\left[\rho_{h}-\rho\right] u d \mathbf{x} .
$$

Using now Hölder inequality together with Poincare-Firederichs inequality we find,

$$
|V| \leq\left\|\rho-\rho_{h}\right\|_{-1, \infty, \mathcal{T}_{h}}\|u\|_{W^{1,1}\left(\Omega_{\mathbf{x}}\right) / \mathbb{R}}
$$

We now estimate the term $\|u\|_{W^{1,1}\left(\Omega_{\mathbf{x}}\right)}$. Sobolev's imbeddings together with the a-priori estimate (78) for problem (87) give

$$
\begin{aligned}
\|u\|_{W^{1,1}\left(\Omega_{\mathbf{x}}\right)} & \leq C\|u\|_{W^{1, p}\left(\Omega_{\mathbf{x}}\right)} \leq \frac{C}{(p-1)}\left\|\operatorname{div} \Pi_{h}^{k} \boldsymbol{\psi}\right\|_{W^{-1, p}\left(\Omega_{\mathbf{x}}\right)} \leq \frac{C}{(p-1)}\left\|\Pi_{h}^{k} \boldsymbol{\psi}\right\|_{L^{p}\left(\Omega_{\mathbf{x}}\right)} \\
& \leq \frac{C}{(p-1)} h^{-d\left(\frac{1}{2}-\frac{1}{p}\right)}\left\|\Pi_{h}^{k} \boldsymbol{\psi}\right\|_{0, \Omega_{\mathbf{x}}} \leq \frac{C}{(p-1)} h^{d / 2} h^{-d\left(1-\frac{1}{p}\right)}\|\boldsymbol{\psi}\|_{0, \Omega_{\mathbf{x}}} \\
& \leq \frac{C}{(p-1)} h^{-d\left(1-\frac{1}{p}\right)} h^{d / 2}\left\|\Pi_{h}^{k}(\mathbf{E})-\mathbf{E}_{h}\right\|_{\sigma^{-2(\alpha+d)}},
\end{aligned}
$$

where we have also used inverse inequality, the $L^{2}$-stability of the Raviart-Thomas projection together with the definition of $\boldsymbol{\psi}$. Taking now $p=1+1 /(\log (1 / h))$ and using the fact that $h^{-d|\log (h)|^{-1}}=O(1)$, we finally have

$$
\|u\|_{W^{1,1}\left(\Omega_{\mathbf{x}}\right)} \leq C h^{d / 2}|\log (h)|\left\|\Pi_{h}^{k}(\mathbf{E})-\mathbf{E}_{h}\right\|_{\sigma^{-2(\alpha+d)}} .
$$

Now, from the relations between the weighted norms and the $L^{\infty}$-norms (82) and (81) it follows that

$$
\begin{aligned}
\|u\|_{W^{1,1}\left(\Omega_{\mathbf{x}}\right)} & \leq C h^{d / 2}|\log (h)|\left\|\Pi_{h}^{k}(\mathbf{E})-\mathbf{E}_{h}\right\|_{\sigma^{-2(\alpha+d)}} \\
& \leq C|\log (h)| h^{d / 2} \theta^{-d / 2-\alpha}\left\|\Pi_{h}^{k}(\mathbf{E})-\mathbf{E}_{h}\right\|_{0, \infty, \Omega_{\mathbf{x}}}
\end{aligned}
$$

Substituting the above estimate into (88) we have

$$
|V| \leq C|\log (h)|(h / \theta)^{d / 2} \theta^{-\alpha}\left\|\rho-\rho_{h}\right\|_{-1, \infty, \mathcal{T}_{h}}\left\|\Pi_{h}^{k}(\mathbf{E})-\mathbf{E}_{h}\right\|_{0, \infty, \Omega_{\mathbf{x}}} .
$$

Inserting this estimate into (85) and choosing $C^{*}=\theta / h$ large enough to absorb into the left hand side the terms $\left\|\mathcal{R}_{h}^{k}(\mathbf{E})-\mathbf{E}_{h}\right\|_{\sigma^{-(d+\alpha)}}^{2}$ we get,

$$
\begin{aligned}
\left\|\mathcal{R}_{h}^{k}(\mathbf{E})-\mathbf{E}_{h}\right\|_{\sigma^{-(d+\alpha)}}^{2} & \leq C\left\|\mathcal{R}_{h}^{k}(\mathbf{E})-\mathbf{E}\right\|_{\sigma^{-(d+\alpha)}}^{2} \\
& +C|\log (h)|(h / \theta)^{d / 2} \theta^{-\alpha}\left\|\rho-\rho_{h}\right\|_{-1, \infty, \mathcal{T}_{h}}\left\|\Pi_{h}^{k}(\mathbf{E})-\mathbf{E}_{h}\right\|_{0, \infty, \Omega_{\mathbf{x}}}
\end{aligned}
$$

Using now (81) and (84) to transform the above norms into $L^{\infty}$-norms together with the definition of $\theta$, we finally get

$$
\begin{aligned}
& \left\|\mathcal{R}_{h}^{k}(\mathbf{E})-\mathbf{E}_{h}\right\|_{0, \infty, \Omega_{\mathbf{x}}}^{2} \leq C(\theta / h)^{d}\left\|\mathcal{R}_{h}^{k}(\mathbf{E})-\mathbf{E}\right\|_{0, \infty, \Omega_{\mathbf{x}}}^{2} \\
& \quad+C\left(\theta^{(d+\alpha)} / h^{d}\right)|\log (h)|(h / \theta)^{d / 2} \theta^{-\alpha}\left\|\rho-\rho_{h}\right\|_{-1, \infty, \mathcal{T}_{h}}\left\|\Pi_{h}^{k}(\mathbf{E})-\mathbf{E}_{h}\right\|_{0, \infty, \Omega_{\mathbf{x}}} \\
& \quad \leq C\left\|\mathcal{R}_{h}^{k}(\mathbf{E})-\mathbf{E}\right\|_{0, \infty, \Omega_{\mathbf{x}}}^{2}+\left(C^{*}\right)^{d / 2}|\log (h)|\left\|\rho-\rho_{h}\right\|_{-1, \infty, \mathcal{T}_{h}}\left\|\Pi_{h}^{k}(\mathbf{E})-\mathbf{E}_{h}\right\|_{0, \infty, \Omega_{\mathbf{x}}} .
\end{aligned}
$$


Recalling that $C^{*} \geq 1$ is a constant, the above estimate readily implies the assertion of the lemma and the proof is concluded.

A.4.2. $\boldsymbol{L}^{\infty}$-error estimates for the $L D G$ approximation to the electrostatic field. In [19] the author carries out the pointwise error analysis for the LDG method, with a different approach to that used in [28]. He follows the technique introduced in $[38,37]$, in which instead of using global weighted $L^{2}$ error estimates, one has to use local $L^{2}$ error estimates along with dyadic decompositions of the domain $\Omega_{\mathbf{x}}$. This strategy relies on sharp pointwise bounds for high-order derivatives of the Green's function. These types of the Green's function estimates are well known for smooth domains, but do not hold for general convex polyhedral domains ${ }^{1}$ We wish to note that since we consider periodic boundary conditions, the issue of a-priori estimates reduces to the classical interior a-priori estimates (no special treatment of the boundary is required).

The proof of Lemma 6 follows by modifying one step in the proof of [19, Theorem 4.1] in order to account for the nonlinearity of the Poisson problem (3). But, unlike it happened with Lemma 4, the modification is much more involved and we have to prove some other results that were not provided in [19] by the author. As mentioned before, we argue similarly as in [38, 37], but the fact that we deal with mixed formulation and discontinuous finite elements precludes from a straightforward application of those results.

Prior to show Lemma 6, we introduce some notation that will allow us to use the results already proved in [19]. We wish to stress that although in that work the author deals with the Dirichlet problem, all the error estimates proved in [19] carry over for the periodic Poisson problem.

For each fixed point $\mathbf{z} \in \overline{\Omega_{\mathbf{x}}}$, real number $s$ and arbitrary $\mathbf{x} \in \mathbb{R}^{d}$ consider the weight function

$$
\sigma_{z, h}^{s}(x):=\left(\frac{h}{|z-x|+h}\right)^{s} \quad x, z \in \Omega_{\mathbf{x}}, \quad-\infty<s<\infty .
$$

We consider the following norm notation introduced in [19]

$$
\begin{aligned}
\|\boldsymbol{\tau}\|_{L^{p}(D), z, s} & =\left\|\sigma_{z, h}^{s} \boldsymbol{\tau}\right\|_{L^{p}(D)}, \\
\|\boldsymbol{\tau}\|_{a, 1, D, z, s} & =\|\boldsymbol{\tau}\|_{L^{1}(D), z, s}+\sum_{e \in \mathcal{E}_{h}^{0}} \int_{e \cap D} h \sigma_{z, h}^{s}|\llbracket \boldsymbol{\tau} \rrbracket| d s_{x}, \\
|q|_{c, 1, D, z, s} & =\sum_{e \in \mathcal{E}_{h}} \int_{e \cap D} \sigma_{z, h}^{s}|\llbracket q \rrbracket| d s_{x} .
\end{aligned}
$$

Following [37] we note that if $s>0$ and $|z-x|=O(h)$ then $\sigma_{z, h}^{s}(x)=O(1)$ while $\sigma_{z, h}^{s}(x)=O\left(h^{s}\right)$ when $|z-x|=O(1)$. Obviously for $s=0$ we recover

\footnotetext{
${ }^{1}$ Recently, in [31], the authors have shown Hölder type estimates for the first order derivatives and the second order mixed derivatives of the Green's function, which allows to provide pointwise and $L^{\infty}$-estimates in general polygonal domains.
} 
the norms without weights. Also we note that the denominator in (89) could be replaced by $\left(|z-x|^{2}+h^{2}\right)^{1 / 2}$ without affecting the results. Notice however, that positive powers of this weight correspond to negative powers of the weight function defined in (79).

We also define following [17]

$$
\left|q\left\|_{W_{h}^{1,1}(D)}=\right\| q\left\|_{L^{1}(D)}+\sum_{T^{x} \in \mathcal{T}_{h_{x}}^{x}}\right\| \nabla q \|_{L^{1}\left(T^{x} \cap D\right)}+\sum_{e \in \mathcal{E}_{h}} \int_{e \cap D}\right| \llbracket q \rrbracket \mid d s_{x} .
$$

We shall

Proof of Lemma 6. Observe that substracting (30) from the mixed formulation of the continuous Poisson problem 3, we have the error equations

$$
\begin{cases}a\left(\mathbf{E}-\mathbf{E}_{h}, \boldsymbol{\tau}\right)+b\left(\boldsymbol{\tau}, \Phi-\Phi_{h}\right)=0 & \forall \boldsymbol{\tau} \in \mathbf{\Xi}_{h}^{r}, \\ -b\left(\mathbf{E}-\mathbf{E}_{h}, q\right)+c\left(\Phi-\Phi_{h}, q\right)=F(q) & \forall q \in Q_{h}^{r},\end{cases}
$$

where $F(q)=\int_{\Omega_{\mathbf{x}}}\left(\rho-\rho_{h}\right) q d \mathbf{x} \forall q \in Q_{h}^{r}$.

Let now $T_{z} \in \mathcal{T}_{h_{x}}^{x}$ be such that $z \in \bar{T}_{z}$ and let $\boldsymbol{\delta}_{\mathbf{z}} \in \mathcal{C}^{\infty}\left(\Omega_{\mathbf{x}}\right)^{d}$ be a regularization of the Dirac mass satisfying the following properties:

$\operatorname{supp}\left(\boldsymbol{\delta}_{\boldsymbol{z}}\right) \subset \bar{T}_{z}, \quad \mathbf{E}_{h}(z)=\int_{\Omega_{\mathbf{x}}} \mathbf{E}_{h} \cdot \boldsymbol{\delta}_{\boldsymbol{z}} d \mathbf{x}, \quad\left\|\boldsymbol{\delta}_{\mathbf{z}}\right\|_{L^{p}\left(\Omega_{\mathbf{x}}\right)} \leq C h^{-d\left(1-\frac{1}{p}\right)} \quad 1 \leq p \leq \infty$.

Using triangle inequality and (92), we have

$$
\left|\left(\mathbf{E}-\mathbf{E}_{h}\right)(z)\right| \leq\left\|\mathbf{E}-\mathcal{P}_{h}(\mathbf{E})\right\|_{L^{\infty}\left(\Omega_{\mathbf{x}}\right), z, s}+\left|\int_{\Omega_{\mathbf{x}}} \boldsymbol{\delta}_{\mathbf{z}}\left(\mathbf{E}-\mathbf{E}_{h}\right) d \mathbf{x}\right|
$$

Next, we introduce the regularized Green's function. Let $\tilde{g}_{z} \in H_{\mathrm{per}}^{1}\left(\Omega_{\mathbf{x}}\right) \cap L_{0}^{2}\left(\Omega_{\mathbf{x}}\right)$ be the solution of

$$
-\Delta \tilde{g}_{z}=\nabla \cdot\left(\boldsymbol{\delta}_{\mathbf{z}}\right)-\mathfrak{c}_{0}, \quad \mathfrak{c}_{0}:=\int_{\Omega_{\mathbf{x}}} \nabla \cdot\left(\boldsymbol{\delta}_{\mathbf{z}}\right) d \mathbf{x},
$$

and let $\widetilde{\mathbf{G}}_{z}:=\nabla \tilde{g}_{z}+\boldsymbol{\delta}_{\mathbf{z}}$ so that $-\nabla \cdot \widetilde{\mathbf{G}}_{z}=-\mathfrak{c}_{0}$. The problem is completed by imposing periodic boundary conditions for both $\tilde{g}_{z}$ and $\widetilde{\mathbf{G}}_{z}$.

Let now $\left(\widetilde{\mathbf{G}}_{z, h}, \tilde{g}_{z, h}\right)$ be the DG approximation to $\left(\widetilde{\mathbf{G}}_{z}, \tilde{g}_{z}\right)$ that satisfies

$$
\begin{array}{rlrl}
a\left(\widetilde{\mathbf{G}}_{z}-\widetilde{\mathbf{G}}_{z, h}, \boldsymbol{\tau}\right)+b\left(\boldsymbol{\tau}, \tilde{g}_{z}-\tilde{g}_{z, h}\right) & =0 & & \forall \boldsymbol{\tau} \in \boldsymbol{\Xi}_{h}^{r}, \\
-b\left(\widetilde{\mathbf{G}}_{z}-\widetilde{\mathbf{G}}_{z, h}, q\right)+c\left(\tilde{g}_{z}-\tilde{g}_{z, h}, q\right) & =0 & \forall q \in Q_{h}^{r} .
\end{array}
$$

From [19, Lemma 4.1] and [19, Lemma 4.2], respectively, we have the estimates:

$$
\begin{array}{r}
\left\|\nabla\left(\tilde{g}_{z}-\mathcal{P}_{\mathbf{x}}\left(\tilde{g}_{z}\right)\right)\right\|_{L^{1}(\Omega), z,-s}+h\left\|\nabla \cdot\left(\widetilde{\mathbf{G}}_{z}-\mathcal{P}_{h}\left(\widetilde{\mathbf{G}}_{z}\right)\right)\right\|_{L^{1}\left(\Omega_{\mathbf{x}}\right), z,-s} \leq C|\log (h)|^{\bar{r}}, \\
\left\|\tilde{g}_{z}-\tilde{g}_{z, h}\right\|_{c, 1, \Omega, z,-s}+\left\|\widetilde{\mathbf{G}}_{z}-\widetilde{\mathbf{G}}_{z, h}\right\|_{L^{1}(\Omega), z,-s} \leq C|\log (h)|^{\bar{r}},
\end{array}
$$

where $\bar{r}=0$ for $0 \leq s<r-1$ and $\bar{r}=1$ for $s=r-1$. 
Next, observe that the solution $\left(\widetilde{\mathbf{G}}_{z}, \tilde{g}_{z}\right)$ satisfies

$$
\begin{aligned}
a\left(\widetilde{\mathbf{G}}_{z}, \boldsymbol{\tau}\right)+b\left(\boldsymbol{\tau}, \tilde{g}_{z}\right) & =\int_{\Omega_{\mathbf{x}}} \boldsymbol{\delta}_{\mathbf{z}} \boldsymbol{\tau} d \mathbf{x} & \forall \boldsymbol{\tau} \in H\left(\operatorname{div} ; \Omega_{\mathbf{x}}\right), \\
-b\left(\widetilde{\mathbf{G}}_{z}, q\right)+c\left(\tilde{g}_{z}, q\right) & =0 & \forall q \in L_{0}^{2}\left(\Omega_{\mathbf{x}}\right) .
\end{aligned}
$$

Observe that in the last equation above we have used that since $c_{0}$ is constant $\left(c_{0}, q\right)=0$ for all $q \in L_{0}^{2}(\Omega)$.

By setting now $(\boldsymbol{\tau}, q)=\left(\mathbf{E}-\mathbf{E}_{h}, \Phi-\Phi_{h}\right)$ in $(97)$ and $(\boldsymbol{\tau}, q)=\left(\widetilde{\mathbf{G}}_{z, h}, \tilde{g}_{z, h}\right)$ in (91) and combining both equations we get

$$
\begin{aligned}
\int_{\Omega_{\mathbf{x}}}\left(\mathbf{E}-\mathbf{E}_{h}\right) \boldsymbol{\delta}_{\mathbf{z}} d \mathbf{x}= & a\left(\widetilde{\mathbf{G}}_{z}, \mathbf{E}-\mathbf{E}_{h}\right)+b\left(\mathbf{E}-\mathbf{E}_{h}, \tilde{g}_{z}\right) \\
= & a\left(\widetilde{\mathbf{G}}_{z}-\widetilde{\mathbf{G}}_{z, h}, \mathbf{E}-\mathbf{E}_{h}\right)+b\left(\mathbf{E}-\mathbf{E}_{h}, \tilde{g}_{z}-\tilde{g}_{z, h}\right) \\
& -b\left(\widetilde{\mathbf{G}}_{z}-\widetilde{\mathbf{G}}_{z, h}, \Phi-\Phi_{h}\right)+c\left(\Phi-\Phi_{h}, \tilde{g}_{z}-\tilde{g}_{z, h}\right)+F\left(\tilde{g}_{z, h}\right) \\
= & a\left(\widetilde{\mathbf{G}}_{z}-\widetilde{\mathbf{G}}_{z, h}, \mathbf{E}-\mathcal{P}_{h}(E)\right)+b\left(\mathbf{E}-\mathcal{P}_{h}(E), \tilde{g}_{z}-\tilde{g}_{z, h}\right) \\
& +b\left(\widetilde{\mathbf{G}}_{z}-\widetilde{\mathbf{G}}_{z, h}, \mathcal{P}_{h}(\Phi)-\Phi\right)+c\left(\Phi-\mathcal{P}_{h}(\Phi), \tilde{g}_{z}-\tilde{g}_{z, h}\right)+F\left(\tilde{g}_{z, h}\right) \\
& =I_{1}+I_{2}+I_{3}+I_{4}+F\left(\tilde{g}_{z, h}\right)
\end{aligned}
$$

where in the last step we have used the Galerkin orthogonality given in (94). Then, the first four terms are estimated exactly as in [19, Theorem 4.1];

$$
\begin{aligned}
I_{1} \leq & \left\|\widetilde{\mathbf{G}}_{z}-\widetilde{\mathbf{G}}_{z, h}\right\|_{L^{1}\left(\Omega_{\mathbf{x}}\right), z,-s}\left\|\mathbf{E}-\mathcal{P}_{h}(E)\right\|_{L^{\infty}\left(\Omega_{\mathbf{x}}\right), z, s} \\
I_{2} \leq & h^{-1}\left\|\Phi-\mathcal{P}_{h}(\Phi)\right\|_{L^{\infty}\left(\Omega_{\mathbf{x}}\right), z, s}\left\|\tilde{g}_{z}-\tilde{g}_{z, h}\right\|_{c, 1, \Omega_{\mathbf{x}}, z,-s} \\
I_{3} \leq & \left\|\mathbf{E}-\mathcal{P}_{h}(E)\right\|_{L^{\infty}\left(\Omega_{\mathbf{x}}\right), z, s}\left(\left\|\tilde{g}_{z}-\tilde{g}_{z, h}\right\|_{c, 1, \Omega_{\mathbf{x}}, z,-s}+\| \nabla\left(\tilde{g}_{z}-\mathcal{P}_{h}\left(\tilde{g}_{z}\right) \|_{L^{1}\left(\Omega_{\mathbf{x}}\right), z,-s}\right),\right. \\
I_{4} \leq & C h^{-1}\left\|\Phi-\mathcal{P}_{h}(\Phi)\right\|_{L^{\infty}\left(\Omega_{\mathbf{x}}\right), z, s} \\
& \times\left(\left\|\widetilde{\mathbf{G}}_{z}-\widetilde{\mathbf{G}}_{z, h}\right\|_{a, 1, s, z}+h\left\|\nabla \cdot\left(\widetilde{\mathbf{G}}_{z}-\mathcal{P}_{h}\left(\widetilde{\mathbf{G}}_{z}\right)\right)\right\|_{L^{1}\left(\Omega_{\mathbf{x}}\right), z,-s}\right),
\end{aligned}
$$

which in view of (95) and (96) give

$I_{1}+I_{2}+I_{3}+I_{4} \leq C|\log (h)|^{\bar{r}}\left(\left\|\mathbf{E}-\mathcal{P}_{h}(\mathbf{E})\right\|_{L^{\infty}\left(\Omega_{\mathbf{x}}\right), z, s}+h^{-1}\left\|\Phi-\mathcal{P}_{h}(\Phi)\right\|_{L^{\infty}\left(\Omega_{\mathbf{x}}\right), z, s}\right)$.

The passage from the localized estimate to an $L^{\infty}$-estimate can then be achieved by choosing $z \in \Omega_{\mathbf{x}}$ such that $\left|\left(\mathbf{E}-\mathbf{E}_{h}\right)(z)\right|=\left\|\mathbf{E}-\mathbf{E}_{h}\right\|_{0, \infty, \Omega_{\mathbf{x}}}$ and setting $s=0$.

Therefore, we only need to estimate the last term in (98). Triangle inequality and Hölder inequality give

$$
\begin{aligned}
\left|F\left(\tilde{g}_{z, h}\right)\right| & \leq\left|F\left(\tilde{g}_{z}-\tilde{g}_{z, h}\right)\right|+\left|F\left(\tilde{g}_{z}\right)\right| \\
& \leq\|F\|_{W^{-1, \infty}\left(\mathcal{T}_{h_{x}}^{x}\right)}\left\|\tilde{g}_{z}-\tilde{g}_{z, h}\right\|_{W_{h}^{1,1}\left(\mathcal{T}_{h_{x}}^{x}\right)}+\|F\|_{W^{-1, \infty}\left(\Omega_{\mathbf{x}}\right)}\left\|\tilde{g}_{z}\right\|_{W^{1,1}\left(\Omega_{\mathbf{x}}\right)} .
\end{aligned}
$$

Hence, to conclude we need to bound the above terms involving the generalized green function $\tilde{g}_{z}$. Last term in (99) can be estimated arguing as in [38, Lemma 
2.2] (or [37, proof of Lemma 1.8]). Sobolev's imbeddings together with the apriori estimate (78) for problem (93) and the bound (92) give for $1<p \leq 2$ $(100)$

$$
\left\|\tilde{g}_{z}\right\|_{W^{1,1}\left(\Omega_{\mathbf{x}}\right)} \leq C\left\|\tilde{g}_{z}\right\|_{W^{1, p}\left(\Omega_{\mathbf{x}}\right)} \leq \frac{C}{p-1}\left\|\boldsymbol{\delta}_{\mathbf{z}}\right\|_{L^{p}\left(T_{z}\right)} \leq \frac{C}{p-1} h^{-d\left(1-\frac{1}{p}\right)} \leq C|\log (h)|,
$$

where in last step we have taken $p=1+1 /(\log (1 / h))$ and used the fact that $h^{-d|\log (h)|^{-1}}=O(1)$.

Now we estimate the first term in (99). Let $E_{g}=\tilde{g}_{z}-\tilde{g}_{z, h}$ and let $\mathbf{T}_{\mathbf{g}}=$ $\nabla_{\mathbf{x}}^{h}\left(\tilde{g}_{z}-\tilde{g}_{z, h}\right)$. From the definition in (90), we have

$$
\left\|\tilde{g}_{z}-\tilde{g}_{z, h}\right\|_{W_{h}^{1,1}\left(\mathcal{T}_{h_{x}}^{x}\right)}=\left\|E_{g}\right\|_{L^{1}\left(\Omega_{\mathbf{x}}\right)}+\sum_{T^{x} \in \mathcal{T}_{h_{x}}^{x}}\left\|\mathbf{T}_{\mathbf{g}}\right\|_{L^{1}\left(T^{x}\right)}+\sum_{e \in \mathcal{E}_{x}} \int_{e}\left|\llbracket E_{g} \rrbracket\right| d s_{x}
$$

Last term above is estimated by setting $s=0$ in the estimate (96). We next estimate the second term above. We first recall that for each $T^{x} \in \mathcal{T}_{h_{x}}^{x}$

$$
\left\|\mathbf{T}_{\tilde{g}}\right\|_{L^{1}\left(T^{x}\right)}=\sup _{\substack{\boldsymbol{\tau} \in \mathcal{C}_{0}^{\infty}\left(T^{x}\right) \\\|\boldsymbol{\tau}\|_{L^{\infty}\left(T^{x}\right)}=1}}\left(\int_{T^{x}} \mathbf{T}_{\tilde{g}} \cdot \boldsymbol{\tau} d \mathbf{x}\right)=\left(\int_{T^{x}} \mathbf{T}_{\tilde{g}} \cdot \boldsymbol{\tau}_{T}^{\epsilon} d \mathbf{x}\right)-\epsilon \quad \epsilon>0
$$

for some $\boldsymbol{\tau}_{T}^{\epsilon} \in \mathcal{C}_{0}^{\infty}\left(T^{x}\right)$ with $\left\|\tau^{\epsilon}\right\|_{0, \infty, T^{x}}=1$. Let $\boldsymbol{\tau}^{\epsilon}:=\sum_{T} \boldsymbol{\tau}_{T}^{\epsilon} \in \mathcal{C}_{0}^{\infty}(\Omega)$, be the function such that $\left.\boldsymbol{\tau}^{\epsilon}\right|_{T^{x}}=\boldsymbol{\tau}_{T}^{\epsilon}$. Hence, summation over all the elements in $\mathcal{T}_{h_{x}}^{x}$ gives,

$$
\begin{aligned}
\sum_{T^{x} \in \mathcal{T}_{h_{x}}^{x}}\left\|\mathbf{T}_{\tilde{g}}\right\|_{L^{1}\left(T^{x}\right)}+\sum_{T^{x} \in \mathcal{T}_{h_{x}}^{x}} \epsilon & =\sum_{T^{x} \in \mathcal{T}_{h_{x}}^{x}}\left(\int_{T^{x}} \nabla_{\mathbf{x}}\left(\tilde{g}_{z}-\tilde{g}_{z, h}\right) \cdot \boldsymbol{\tau}_{T^{x}}^{\epsilon} d \mathbf{x}\right) \\
& =\int_{\Omega^{\mathbf{x}}} \nabla_{\mathbf{x}}^{h}\left(\tilde{g}_{z}-\tilde{g}_{z, h}\right) \cdot \boldsymbol{\tau}^{\epsilon} d \mathbf{x} .
\end{aligned}
$$

Notice also that summing and substracting $\mathcal{P}_{\mathbf{x}}\left(\tau^{\epsilon}\right)$ (with $\mathcal{P}_{\mathbf{x}}$ denoting the standard local $L^{2}$-projection) we have

$\sum_{T^{x} \in \mathcal{T}_{h x}^{x}}\left\|\mathbf{T}_{\tilde{g}}\right\|_{L^{1}\left(T^{x}\right)}+\sum_{T^{x} \in \mathcal{T}_{h x}^{x}} \epsilon=\int_{\Omega^{\mathbf{x}}} \mathbf{T}_{\tilde{g}} \cdot \mathcal{P}_{\mathbf{x}}\left(\boldsymbol{\tau}^{\epsilon}\right) d \mathbf{x}+\int_{\Omega^{\mathbf{x}}} \mathbf{T}_{\tilde{g}} \cdot\left[\boldsymbol{\tau}^{\epsilon}-\mathcal{P}_{\mathbf{x}}\left(\boldsymbol{\tau}^{\epsilon}\right)\right] d \mathbf{x}=S_{1}+S_{2}$

We now estimate each of the above terms. For the first one, using the definition $(31)$ of the bilinear form $b(\cdot, \cdot)$ together with the first error equation in (94), 
we have

$$
\begin{aligned}
\int_{\Omega} \mathbf{T}_{\tilde{g}} \cdot \mathcal{P}_{\mathbf{x}}\left(\boldsymbol{\tau}^{\epsilon}\right) d \mathbf{x} & =b\left(\mathcal{P}_{\mathbf{x}}\left(\boldsymbol{\tau}^{\epsilon}\right), E_{g}\right) \\
& +\int_{\mathcal{E}_{x}^{0}} \llbracket E_{g} \rrbracket \cdot\left(\left\{\mathcal{P}_{\mathbf{x}}\left(\boldsymbol{\tau}^{\epsilon}\right)\right\}-\mathbf{C}_{12} \llbracket \mathcal{P}_{\mathbf{x}}\left(\boldsymbol{\tau}^{\epsilon}\right) \rrbracket\right) d s_{x}+\int_{\mathcal{E}_{x}^{\partial}} E_{g} \mathcal{P}_{\mathbf{x}}\left(\boldsymbol{\tau}^{\epsilon}\right) \cdot \mathbf{n} d s_{x} \\
& =-a\left(\widetilde{\mathbf{G}}_{z}-\widetilde{\mathbf{G}}_{z, h}, \mathcal{P}_{\mathbf{x}}\left(\boldsymbol{\tau}^{\epsilon}\right)\right) \\
& +\int_{\mathcal{E}_{x}^{0}} \llbracket E_{g} \rrbracket \cdot\left(\left\{\mathcal{P}_{\mathbf{x}}\left(\boldsymbol{\tau}^{\epsilon}\right)\right\}-\mathbf{C}_{12} \llbracket \mathcal{P}_{\mathbf{x}}\left(\boldsymbol{\tau}^{\epsilon}\right) \rrbracket\right) d s_{x}+\int_{\mathcal{E}_{x}^{\partial}} E_{g} \mathcal{P}_{\mathbf{x}}\left(\boldsymbol{\tau}^{\epsilon}\right) \cdot \mathbf{n} d s_{x}
\end{aligned}
$$

Hence, arguing as in [19, Proof of Theorem 3.1], Hölder inequality, the definitions of the norms (90) together with estimate (96) from [19, Lemma 4.2] with $s=0$ give

$\left|S_{1}\right| \leq\left\|\mathcal{P}_{\mathbf{x}}\left(\boldsymbol{\tau}^{\epsilon}\right)\right\|_{0, \infty, \mathcal{T}_{h_{x}}^{x}}\left(\left\|\widetilde{\mathbf{G}}_{z}-\widetilde{\mathbf{G}}_{z, h}\right\|_{L^{1}(\Omega)}+\left\|\tilde{g}_{z}-\tilde{g}_{z, h}\right\|_{c, 1, \Omega}\right) \leq C|\log (h)|^{\bar{r}}\left\|\boldsymbol{\tau}^{\epsilon}\right\|_{0, \infty, \mathcal{T}_{h_{x}}^{x}}$,

where in the last step we have also used the $L^{\infty}$-stability of the $L^{2}$-projection. In the above estimate, $\bar{r}=1$ for $r=1$ and $\bar{r}=0$ for $r>1$. We now estimate the second term in (102). From the definition of the standard $L^{2}$-projection, we have

$$
\begin{aligned}
& \sum_{T^{x} \in \mathcal{T}_{h_{x}}^{x}} \int_{T^{x}}\left(\nabla \tilde{g}_{z}-\nabla \tilde{g}_{z, h}\right) \cdot\left[\boldsymbol{\tau}_{T^{x}}^{\epsilon}-\mathcal{P}_{\mathbf{x}}\left(\boldsymbol{\tau}_{T^{x}}^{\epsilon}\right)\right] d \mathbf{x} \\
&=\sum_{T^{x} \in \mathcal{T}_{h_{x}}^{x}} \int_{T^{x}} \nabla\left(\tilde{g}_{z}-\mathcal{P}_{\mathbf{x}}\left(\tilde{g}_{z}\right)\right) \cdot\left[\boldsymbol{\tau}_{T^{x}}^{\epsilon}-\mathcal{P}_{\mathbf{x}}\left(\boldsymbol{\tau}_{T^{x}}^{\epsilon}\right)\right] d \mathbf{x} .
\end{aligned}
$$

Hence, Hölder inequality, estimate (95) from [19, Lemma 4.1] with $s=0$ and the $L^{\infty}$-stability of the $L^{2}$-projection yield to

$$
\left|S_{2}\right| \leq\left\|\nabla\left(\tilde{g}_{z}-\mathcal{P}_{\mathbf{x}}\left(\tilde{g}_{z}\right)\right)\right\|_{L^{1}\left(\mathcal{T}_{h}\right)}\left\|\boldsymbol{\tau}^{\epsilon}-\mathcal{P}_{\mathbf{x}}\left(\boldsymbol{\tau}^{\epsilon}\right)\right\|_{0, \infty, \mathcal{T}_{h_{x}}^{x}} \leq C|\log (h)|^{\bar{r}}\left\|\boldsymbol{\tau}^{\epsilon}\right\|_{0, \infty, \mathcal{T}_{h_{x}}^{x}},
$$

where as before $\bar{r}=1$ for $r=1$ and $\bar{r}=0$ for $r>1$. Thus, substituting the above estimate together with (103) in (102) we have,

$$
\sum_{T^{x} \in \mathcal{T}_{h_{x}}^{x}}\left\|\mathbf{T}_{\tilde{g}}\right\|_{L^{1}\left(T^{x}\right)}+\sum_{T^{x} \in \mathcal{T}_{h_{x}}^{x}} \epsilon \leq 2 C|\log (h)|^{\bar{r}}\left\|\boldsymbol{\tau}^{\epsilon}\right\|_{0, \infty, \mathcal{T}_{h_{x}}^{x}}=2 C|\log (h)|^{\bar{r}},
$$

and now letting $\epsilon \downarrow 0$ we finally get

$$
\sum_{T^{x} \in \mathcal{T}_{h_{x}}^{x}}\left\|\nabla\left(\tilde{g}_{z}-\tilde{g}_{z, h}\right)\right\|_{L^{1}\left(T^{x}\right)} \leq 2 C|\log (h)|^{\bar{r}} .
$$

Hence, to conclude we need to provide a bound for $\left\|\tilde{g}_{z}-\tilde{g}_{z, h}\right\|_{L^{1}\left(\Omega_{\mathbf{x}}\right)}$. Using the fact that $\Omega_{\mathrm{x}}$ is convex and both $\tilde{g}_{z}$ and $\tilde{g}_{z, h}$ are functions with zero average over $\Omega_{\mathbf{x}}$, triangle inequality together with the $L^{1}$-Poincaré-Friederichs inequality for 
$W^{1, p}\left(\Omega_{\mathbf{x}}\right)$ functions [1] and the $L^{1}$-Poincaré-Friederichs inequality for DG functions [17], we have

$$
\begin{aligned}
\left\|\tilde{g}_{z}-\tilde{g}_{z, h}\right\|_{L^{1}\left(\Omega_{\mathbf{x}}\right)} & \leq\left\|\tilde{g}_{z}\right\|_{L^{1}\left(\Omega_{\mathbf{x}}\right)}+\left\|\tilde{g}_{z, h}\right\|_{L^{1}\left(\Omega_{\mathbf{x}}\right)} \\
& \leq \frac{\operatorname{diam}\left(\Omega_{\mathbf{x}}\right)}{2}\left\|\nabla \tilde{g}_{z}\right\|_{L^{1}\left(\Omega_{\mathbf{x}}\right)}+C\left(\left\|\nabla \tilde{g}_{z, h}\right\|_{L^{1}\left(\Omega_{\mathbf{x}}\right)}+\left\|\tilde{g}_{z, h}\right\|_{c, 1, \Omega_{\mathbf{x}}}\right) \\
& \leq C|\log (h)|+C|\log (h)|^{\bar{r}} \leq C|\log (h)|,
\end{aligned}
$$

where in the last step we have also used the bounds (100) together with (104) and (96).

Therefore substituting the above estimate together with the bounds (104) and (96) into (101), we finally get

$$
\left\|\tilde{g}_{z}-\tilde{g}_{z, h}\right\|_{W_{h}^{1,1}\left(\mathcal{T}_{h}\right)} \leq C|\log (h)|,
$$

which together with (100), (99) and the definition of the functional $F$ concludes the proof of the lemma.

\section{Appendix B. Proofs of Lemmas 8 and 9}

In this appendix we provide the proofs of Lemmas 8 and Lemma 9 from $\S 4$. To ease the presentation (and simplify the notations), we give detailed proofs for the case $d=2$, since the differences and difficulties compared to $d=1$ are already present in that case. The case $d=3$ is treated analogously and therefore it is omitted.

We start by introducing some further notation that will be used in the proofs. Note that for any $R=T^{x} \times T^{v} \in \mathcal{T}_{h}$, both $T^{x}$ and $T^{v}$ are $d$-rectangles and so the outward unit normal $\mathbf{n}$ at $\partial T^{x} \times T^{v}$ (resp. at $\left.T^{x} \times \partial T^{v}\right)$ is a simple coordinate vector in the $\mathbf{x}$-plane (resp. in the $\mathbf{v}$-plane); $(1,0,0,0)$ or $(0,1,0,0)$ (resp. $(0,0,1,0)$ or $(0,0,0,1))$. Then, according to our notation we use $\mathbf{n}^{-}=\mathbf{n}_{\partial T^{x}}$ to denote the outward unit normal to $T^{x}$ and we denote the inner and outer traces with respect to $\partial T^{x} \times T^{v}$, by $\varphi^{-}$and $\varphi^{+}$, respectively. With a small abuse on the notation, we also designate by $\mathbf{n}^{-}=\mathbf{n}_{\partial T^{v}}$ and $\varphi^{-}$and $\varphi^{+}$will also designate the inner and outer traces with respect to $T^{x} \times \partial T^{v}$. Let $\partial T^{x}=e_{1}^{ \pm} \cup e_{2}^{ \pm}$with $e_{i}^{ \pm}$ denoting the edges of $\partial T^{x}$ in the $x_{i}$-direction and

$$
\text { (105) } e_{i}^{+}=\left\{e \subset \partial T^{x}: \mathbf{v} \cdot \mathbf{n}^{-}>0\right\} \quad e_{i}^{-}=\left\{e \subset \partial T^{x}: \mathbf{v} \cdot \mathbf{n}^{-}<0\right\} \quad i=1,2 .
$$

Similarly $\partial T^{v}=\gamma_{1}^{ \pm} \cup \gamma_{2}^{ \pm}$with $\gamma_{i}^{ \pm}$denoting the edges of $\partial T^{v}$ in the $v_{j}$-direction: (106)

$$
\gamma_{i}^{+}=\left\{\gamma \subset \partial T^{v}: \mathbf{E}_{h} \cdot \mathbf{n}^{-}>0\right\} \quad \gamma_{i}^{-}=\left\{\gamma \subset \partial T^{v}: \mathbf{E}_{h} \cdot \mathbf{n}^{-}<0\right\} \quad i=1,2 .
$$

Next lemma extends [33, Lemma 8] and [21, Lemma 3.6] to higher dimensions also with the more general projections defined in $\S 4.2$.

Lemma 12. Let $\mathcal{T}_{h}=\mathcal{T}_{h_{x}}^{x} \times \mathcal{T}_{h_{v}}^{v}$ be the tensor product of two cartesian meshes $\mathcal{T}_{h_{x}}^{x}$ and $\mathcal{T}_{h_{v}}^{v}$ of $\Omega_{\mathbf{x}}$ and $\Omega_{\mathbf{v}}$, respectively. Let $k \geq 1$ and let $f \in \mathcal{C}^{0}\left([0, T] ; W^{1, \infty}(\Omega) \times\right.$ 
$\left.H^{k+2}(\Omega)\right)$ be the distribution function solution of (1)-(3) and $f_{h} \in \mathcal{Z}_{h}^{k}$ its approximation satisfying $(16)$. For any $R \in \mathcal{T}_{h}$ let $\mathcal{K}_{R}^{1}$ and $\mathcal{K}_{R}^{2}$ be defined as in (46)-(47). Let $\mathbf{v}^{0}=\mathcal{P}_{v}^{0}(\mathbf{v})$ and $\mathbf{E}^{0}=\mathcal{P}_{x}^{0}(\mathbf{E})$ be the local $L^{2}$-projections onto the piecewiseconstants on $\mathcal{T}_{h}$ of $\mathbf{v}$ and $\mathbf{E}$ respectively. Then for any $R=T^{x} \times T^{v} \in \mathcal{T}_{h}$, the following estimate hold

$$
\left|\mathcal{K}_{R}^{1}\left(\mathbf{v}^{0}, f, \varphi\right)\right| \leq C\left|\mathbf{v}^{0}\right| h^{k+1}\|f\|_{k+2, R}\|\varphi\|_{0, R} .
$$

Moreover, if $\mathbf{E}$ does not vanish on $R$ it also holds

$$
\left|\mathcal{K}_{R}^{2}\left(\mathbf{E}^{0}, f, \varphi\right)\right| \leq C\|\mathbf{E}\|_{0, \infty, T^{x}} h^{k+1}\|f\|_{k+2, R}\|\varphi\|_{0, R} .
$$

Furthermore, if one of the components of $\mathbf{E}$ vanishes on $R$, but the other (others) do not i.e., $E_{i}\left(\mathbf{x}^{*}\right)=0$ for some $\mathbf{x}^{*} \in T^{x}$ but $E_{j} \neq 0$ on $R$ for $j \neq i$, then

$$
\left|\mathcal{K}_{R}^{v_{j}}\left(E_{j}^{0}, f, \varphi\right)\right| \leq C\|\mathbf{E}\|_{0, \infty, T^{x}} h^{k+1}\|f\|_{k+2, R}\|\varphi\|_{0, R}
$$

Proof. We prove the lemma for $d=2$. We start by noting that since both $\mathbf{v}^{0}$ and $\mathbf{E}^{0}$ are nonzero constant vectors, without loss of generality we can assume for both that all their components are positive; i.e. $\bar{v}_{1}, \bar{v}_{2}>0$ and respectively $E_{1}^{0}>0$ and $E_{2}^{0}>0$. Then we can further rewrite

$$
\begin{aligned}
\mathcal{K}_{R}^{1}(\overline{\mathbf{v}}, f, \varphi) & =\mathcal{K}_{R}^{x_{1}}(\overline{\mathbf{v}}, f, \varphi)+\mathcal{K}_{R}^{x_{2}}(\overline{\mathbf{v}}, f, \varphi) \\
\mathcal{K}_{R}^{2}\left(\mathbf{E}^{0}, f, \varphi\right) & =\mathcal{K}_{R}^{v_{1}}\left(\mathbf{E}^{0}, f, \varphi\right)+\mathcal{K}_{R}^{v_{2}}\left(\mathbf{E}^{0}, f, \varphi\right),
\end{aligned}
$$

where, using the notation given in (105) and (106) and taking into account the definitions of the numerical fluxes (17) and the projection operator $\widetilde{\Pi}$ the two terms above read

$$
\begin{aligned}
\mathcal{K}_{R}^{x_{i}}(\overline{\mathbf{v}}, f, \varphi)= & \int_{R}\left[f-\Pi_{h}(f)\right] v_{i} \partial_{x_{i}} \varphi d \mathbf{x} d \mathbf{v}-\int_{T^{v}} \int_{e_{j}^{+}}\left[f-\left(\pi_{x, j}^{-} \times \widetilde{\Pi}_{\mathbf{v}}\right)(f)\right]^{-} \varphi \bar{v}_{i} d x_{j} d \mathbf{v} \\
& +\int_{T^{v}} \int_{e_{j}^{-}}\left[f-\left(\pi_{x, j}^{-} \times \widetilde{\Pi}_{\mathbf{v}}\right)(f)\right]^{+} \omega^{h} \bar{v}_{i} d x_{j} d \mathbf{v} \quad i, j=1,2 j \neq i,
\end{aligned}
$$

and

$$
\begin{aligned}
\mathcal{K}_{R}^{v_{i}}\left(\mathbf{E}^{0}, f, \varphi\right)= & \int_{R}\left(f-\Pi_{h}(f)\right) E_{i}^{0} \frac{\partial \varphi}{\partial v_{i}} d \mathbf{x} d \mathbf{v}-\int_{T^{x}} \int_{\gamma_{j}^{+}} E_{i}^{0}\left[f-\left(\widetilde{\Pi}_{\mathbf{x}} \times \widetilde{\Pi}_{\mathbf{v}}\right)(f)\right]^{+} \varphi d v_{j} d \mathbf{x} \\
& +\int_{T^{x}} \int_{\gamma_{j}^{-}} E_{i}^{0}\left[f-\left(\widetilde{\Pi}_{\mathbf{x}} \times \widetilde{\Pi}_{\mathbf{v}}\right)(f)\right]^{-} \varphi d v_{j} d \mathbf{x} \quad i, j=1,2 j \neq i .
\end{aligned}
$$

We now start with the proof of (107). The proof of the approximation result for $\mathcal{K}_{R}^{x_{1}}$ and $\mathcal{K}_{R}^{x_{2}}$ are essentially the same, so we just carry out the proof for one of them, say $\mathcal{K}^{x_{1}}$ :

$$
\begin{aligned}
\mathcal{K}_{R}^{x_{1}}(\overline{\mathbf{v}}, f, \varphi)= & \int_{R}\left[f-\Pi_{h}(f)\right] \bar{v}_{1} \frac{\partial \varphi}{\partial x_{1}} d \mathbf{x} d \mathbf{v}-\int_{T^{v}} \int_{e_{2}^{+}}\left[f-\left(\pi_{x, 2}^{-} \times \widetilde{\Pi}_{\mathbf{v}}\right)(f)\right]^{-} \varphi \bar{v}_{1} d x_{2} d \mathbf{v} \\
& +\int_{T^{v}} \int_{e_{2}^{-}}\left[f-\left(\pi_{x, 2}^{-} \times \widetilde{\Pi}_{\mathbf{v}}\right)(f)\right]^{+} \omega^{h} \bar{v}_{1} d x_{2} d \mathbf{v} .
\end{aligned}
$$


We first consider $\mathcal{K}_{R}^{x_{1}}=\mathcal{K}_{R}^{x_{1}}\left(\mathbf{v}^{0}, f, \varphi\right)$ on the reference element, which with a small abuse on the notation we still denote by $R=T^{x} \times T^{v}=[-1,1]^{4} ; T^{x}=$ $[-1,1]^{2}=T^{v}$. Now, we claim that

$$
\mathcal{K}_{R}^{x_{1}}(\overline{\mathbf{v}}, f, \varphi)=0, \quad \forall f \in \mathbb{P}^{k+1}(R), \quad \varphi \in \mathbb{Q}^{k}(R) .
$$

Let $\varphi \in \mathbb{Q}^{k}(\mathcal{R})$ be fixed and notice that from the fact that $\widetilde{\Pi}$ and $\tilde{\pi}_{x, 2} \times \widetilde{\Pi}_{\mathbf{v}}$ are polynomial preserving operators it follows that (112) holds true for every $f \in$ $\mathbb{Q}^{k}(\mathcal{R})$. Therefore to show $(112)$ it is enough to consider $f \in \mathbb{P}^{k+1}(R) \backslash \mathbb{Q}^{k}(R)$; i.e., $f=x_{1}^{k+1}, x_{2}^{k+1}, v_{1}^{k+1}$ and $v_{2}^{k+1}$.

We first set $f=x_{1}^{k+1}$. Then $f-\Pi_{h}(f)=f-\pi_{x, 1}^{-} f$ on $R$ and so the volume term vanishes by means of (35). As for the boundary terms, note that on $e_{2}^{+}$, $x_{1}=1$ while on $e_{2}^{-}, x_{1}=-1$ and so $f-\Pi_{h}(f)=1-1$ on $e_{2}^{+}$and $f-\Pi_{h}(f)=$ $(-1)^{k+1}-(-1)^{k+1}$ on $e_{2}^{-}$. Hence, $f-\Pi_{h}(f) \equiv 0$ on both boundary integrals.

The other cases $f=x_{2}^{k+1}, v_{1}^{k+1}, v_{2}^{k+1}$ are all done arguing as follows. Integration by parts of the volume term gives:

$$
\begin{aligned}
& \int_{\mathcal{R}} \bar{v}_{1}\left(f-\Pi_{h}(f)\right) \frac{\partial \varphi}{\partial x_{1}} d \mathbf{x} d \mathbf{v}=-\int_{\mathcal{R}} \bar{v}_{1} \frac{\partial\left(f-\Pi_{h}(f)\right)}{\partial x_{1}} \varphi d \mathbf{x} d \mathbf{v} \\
+ & \int_{T^{v}} \int_{e_{2}^{+}} v_{1}\left[f-\left(\pi_{x, 2}^{-} \times \widetilde{\Pi}_{\mathbf{v}}\right)(f)\right]^{-} \varphi^{-} d x_{2} d \mathbf{v}-\int_{T^{v}} \int_{e_{2}^{-}} v_{1}\left[f-\left(\pi_{x, 2}^{+} \times \widetilde{\Pi}_{\mathbf{v}}\right)(f)\right]^{-} \varphi^{-} d x_{2} d \mathbf{v} .
\end{aligned}
$$

Now observe that if $f=x_{2}^{k+1}, v_{1}^{k+1}$ or $v_{2}^{k+1}$ the volume term on the right hand side vanishes (since both $f$ and $\Pi_{h} f$ are independent of $x_{1}$ ). Hence, substituting the above expression into the definition of $\mathcal{K}_{R}^{x_{1}}$ and we get

$$
\begin{aligned}
\mathcal{K}_{R}^{x_{1}}(\overline{\mathbf{v}}, f, \varphi)= & \int_{T^{v}} \int_{e_{2}^{-}}\left[f-\left(\pi_{x, 2}^{-} \times \widetilde{\Pi}_{\mathbf{v}}\right)(f)\right]^{+} \varphi \bar{v}_{1} d x_{2} d \mathbf{v} \\
& -\int_{T^{v}} \int_{e_{2}^{-}} \bar{v}_{1}\left[f-\left(\pi_{x, 2}^{+} \times \widetilde{\Pi}_{\mathbf{v}}\right)(f)\right]^{-} \varphi^{-} d x_{2} d \mathbf{v} \\
= & \int_{T^{v}} \int_{e_{2}^{-}} \bar{v}_{1} \llbracket f-\left(\pi_{x, 2}^{-} \times \widetilde{\Pi}_{\mathbf{v}}\right)(f) \rrbracket_{x_{1}} \varphi^{-} d x_{2} d \mathbf{v}
\end{aligned}
$$

where we have denoted the jump by $\llbracket \cdot \rrbracket_{x_{1}}$ to stress the fact that the jump is taken along the $x_{1}$ direction. If $f=x_{2}^{k+1}$, then $f-\left(\pi_{x, 2}^{-} \times \widetilde{\Pi}_{\mathbf{v}}\right)(f)=f-\pi_{x, 2}^{-}(f)$ which depends only on $x_{2}$ and therefore it is continuous as a function of $x_{1}$. Hence, $\llbracket f-\pi_{x, 2}^{-}(f) \rrbracket_{x_{1}} \equiv 0$.

If $f=v_{1}^{k+1}$ or $f=v_{2}^{k+1}$, the same reasoning ( $f$ independent of $x_{1}$ ) gives $\llbracket f-\widetilde{\Pi}_{v}(f) \rrbracket_{x_{1}} \equiv 0$. Moreover, notice that this does not depend on the precise definition of $\widetilde{\Pi}_{v}$. Even if $\mathbf{E}$ or one of its components happen to vanish inside $R$, one still have $\llbracket f-\mathcal{P}_{v, 1}(f) \rrbracket_{x_{1}}=0$ for $f=v_{1}^{k+1}$ and $\llbracket f-\mathcal{P}_{v, 2}(f) \rrbracket_{x_{1}}=0$ for $f=v_{2}^{k+1}$. Therefore, (112) holds also true for $f \in \mathbb{P}^{k+1}(R)$.

Now, for fixed $\varphi \in \mathbb{Q}^{k}(\mathcal{R})$, the linear functional $f \mapsto \mathcal{K}^{1}(\overline{\mathbf{v}}, f, \varphi)$ is continuous on $H^{m+2}(\mathcal{R})$ with norm bounded by $C \overline{\mathbf{v}}\|\varphi\|_{0, \mathcal{R}}$. Furthermore, due to (112), it 
vanishes over $\mathbb{P}^{m+1}(\mathcal{R})$ for $0 \leq m \leq k$. Thus, from Bramble-Hilbert Lemma, we get that for $f \in H^{m+2}(\mathcal{R})$ with $\mathcal{R}$ being the reference element

$$
\left|\mathcal{K}_{\mathcal{R}}^{x_{1}}(\overline{\mathbf{v}}, f, \varphi)\right| \leq C|\overline{\mathbf{v}}||f|_{m+2, \mathcal{R}}\|\varphi\|_{0, \mathcal{R}} \quad 0 \leq m \leq k
$$

Then, standard scaling arguments together with the $L^{\infty}$-stability of the $L^{2}$ projection yield to the estimate

$$
\left|\mathcal{K}_{\mathcal{R}}^{x_{1}}\left(\overline{\mathbf{v}}, f, \omega^{h}\right)\right| \leq C h^{k+1}\|\mathbf{v}\|_{0, \infty, T^{v}}\|f\|_{k+2, \mathcal{R}}\left\|\omega^{h}\right\|_{0, \mathcal{R}}
$$

The same bound can be shown for $\mathcal{K}_{\mathcal{R}}^{x_{2}}$, and so substituting in (110) we reach (107).

The proofs of estimates (108) and (109) for $\mathcal{K}_{R}^{2}$ are done analogously; if none of the components of $\mathbf{E}$ vanish inside $R$, the definition of the projection operator $\widetilde{\Pi}_{v}$ is given in terms of $\pi_{i, v}^{ \pm}$and so the proof (108) can be performed arguing as for $\mathcal{K}_{R}^{1}$. Hence it is omitted.

In the case one (but not all) of the components of $\mathbf{E}$ vanish we next show that the same argument used for showing (107) can be reproduced in this case.

Without loss of generality we can assume that $E_{1}>0$ (and so $E_{1}^{0}>0$ ) and $E_{2}=0$ (but observe that not necessarily $E_{2}^{0}$ ) in $R$ so that $\widetilde{\Pi}_{v}=\pi_{v, 1}^{+} \times \mathcal{P}_{v, 2}$. We consider $\mathcal{K}_{R}^{v_{1}}$ in the reference element $R=T^{x} \times T^{v}=[-1,1]^{2} \times[-1,1]^{2}$;

$$
\begin{aligned}
\mathcal{K}_{R}^{v_{1}}\left(\mathbf{E}^{0}, f, \varphi\right)= & \int_{R}\left[f-\Pi_{h}(f)\right] E_{1}^{0} \frac{\partial \varphi}{\partial v_{1}} d \mathbf{x} d \mathbf{v}-\int_{T^{x}} \int_{\gamma_{2}^{+}}\left[f-\left(\widetilde{\Pi}_{\mathbf{x}} \times \mathcal{P}_{v, 2}\right)(f)\right]^{+} \varphi E_{1}^{0} d v_{2} d \mathbf{x} \\
& +\int_{T^{x}} \int_{\gamma_{2}^{-}}\left[f-\left(\widetilde{\Pi}_{\mathbf{x}} \times \mathcal{P}_{v, 2}\right)(f)\right]^{-} \varphi E_{1}^{0} d v_{2} d \mathbf{x},
\end{aligned}
$$

and we claim that

$$
\mathcal{K}_{R}^{v_{1}}\left(\mathbf{E}^{0}, f, \varphi\right)=0 \quad \forall f \in \mathbb{P}^{k+1}(R) \quad \forall \varphi \in \mathbb{Q}^{k}(R) .
$$

As before, it is easy to see that (113) holds true for all $f \in \mathbb{Q}^{k}(R)$ using that $\Pi_{h}$ is a polynomial preserving operator. So we only need to check for $f=x_{1}^{k+1}, x_{2}^{k+1}$, $v_{1}^{k+1}, v_{2}^{k+1}$. We start by setting $f=v_{1}^{k+1}$. Then, $f-\Pi_{h} f=f-\widetilde{\Pi}_{x} \times \pi_{v, 1}^{+} \times \mathcal{P}_{v, 2} f$ depends only on $v_{1}$ and therefore taking into account (35), the volume term is zero. As for the boundary terms, since $v_{1}= \pm 1$ on $\gamma_{2}^{ \pm}$and $f$ is a function only of $v_{1}$, from the collocation property (36) of $\pi_{v, 1}^{+}$it can be easily seen that $\left(f-\Pi_{h}(f)\right)_{\left.\right|_{\gamma_{2}^{ \pm}}} \equiv 0$. 
If $f=v_{2}^{k+1}$ (or $f=x_{1}^{k+1}, x_{2}^{k+1}$ ), integration by parts of the volume term, together with the fact that $f-\Pi_{h}(f)$ is independent of $v_{1}$ gives

$$
\begin{aligned}
\mathcal{K}_{R}^{v_{1}}\left(\mathbf{E}^{0}, f, \varphi\right)= & -\int_{R} \frac{\partial\left[f-\Pi_{h}(f)\right]}{\partial v_{1}} E_{1} \varphi d \mathbf{x} d \mathbf{v} \\
& -\int_{T^{x}} \int_{\gamma_{2}^{+}} \llbracket f-\left(\widetilde{\Pi}_{\mathbf{x}} \times \mathcal{P}_{v, 2}\right)(f) \rrbracket_{v_{1}} \varphi E_{1}^{0} d v_{2} d \mathbf{x} \\
= & -\int_{T^{x}} \int_{\gamma_{2}^{+}} \llbracket f-\left(\widetilde{\Pi}_{\mathbf{x}} \times \mathcal{P}_{v, 2}\right)(f) \rrbracket_{v_{1}} \varphi E_{1}^{0} d v_{2} d \mathbf{x}=0
\end{aligned}
$$

where in the last step we have used that for $f=v_{2}^{k+1}$ (resp. $f=x_{i}^{k+1}$ ) the function $f-\mathcal{P}_{v, 2} f$ (resp. $f-\tilde{\pi}_{x, i} f$ ) does not depend on $v_{1}$, and so its jump along this direction is zero. Hence, (113) holds true and now arguing as we did for $\mathcal{K}^{1}$, using Bramble-Hilbert Lemma together with standard scaling arguments, estimate (109) follows and the proof is complete.

Proof of Lemma 8. We prove the Lemma for $d=2$. We show estimate (48) first in a single element $\mathcal{R}=T^{x} \times T^{v} \in \mathcal{T}_{h}$ and then we sum over all elements of the partition. Let $\overline{\mathbf{v}}:=\mathcal{P}_{\mathbf{v}}^{0}(\mathbf{v})$ be the $L^{2}$-projection onto the piecewise constants on $\mathcal{T}_{h}$ of $\mathbf{v}$ and we write

$$
\mathcal{K}_{\mathcal{R}}^{1}\left(\mathbf{v}, f, \omega^{h}\right)=\mathcal{K}_{\mathcal{R}}^{1}\left(\mathbf{v}-\overline{\mathbf{v}}, f, \omega^{h}\right)+\mathcal{K}_{\mathcal{R}}^{1}\left(\overline{\mathbf{v}}, f, \omega^{h}\right) .
$$

Last term above is estimated by means of (107) from Lemma 12. To bound the first term, let $\partial T^{x}=e_{1}^{ \pm} \cup e_{2}^{ \pm}$with $e_{i}^{ \pm}$denoting the edges of $\partial T^{x}$ in the $x_{i}$-direction defined in (105).

Then, Hölder inequality, trace inequality [3] and inverse inequality [20] together with with the error estimates (12) and (42), give

$$
\begin{aligned}
\left|\mathcal{K}_{\mathcal{R}}^{1}\left(\mathbf{v}-\overline{\mathbf{v}}, f, \omega^{h}\right)\right| \leq & \|\mathbf{v}-\overline{\mathbf{v}}\|_{0, \infty, T^{v}} \\
& \times\left(\left\|\omega^{e}\right\|_{0, R}\left\|\nabla_{x} \omega^{h}\right\|_{0, R}+\sum_{i=1,2}\left\|\omega^{e}\right\|_{0, e_{i}^{ \pm} \times T^{v}}\left\|\omega^{h}\right\|_{0, e_{i}^{ \pm} \times T^{v}}\right) \\
\leq & C h_{v} h^{k}\|f\|_{k+1, R}\left\|\omega^{h}\right\|_{0, R} .
\end{aligned}
$$

Substituting this estimate together with (107) in (114) we finally get

$$
\left|\mathcal{K}_{\mathcal{R}}^{1}\left(\mathbf{v}, f, \omega^{h}\right)\right| \leq C h^{k+1}\left(\|f\|_{k+1, \mathcal{R}}+\left\|\mathcal{P}^{0}(\mathbf{v})\right\|_{0, \infty, T^{x}}\|f\|_{k+2, R}\right)\left\|\omega^{h}\right\|_{0, \mathcal{R}},
$$

and so summation over all elements $\mathcal{R} \in \mathcal{T}_{h}$, concludes the proof.

Remark 4. It might seem at first sight that the assumption that the partition $\mathcal{T}_{h}$ is constructed so that $\mathbf{v}$ does not vanish inside any element $R$, has not been used. Notice however, that such condition is implicitly used in the proof, since we implicitly used that both the numerical fluxes and the projections are uniquely defined along each $e_{i} \in \partial T^{x} \times T^{v}$. 
Proof of Lemma 9. We consider first an arbitrary fix element $R=T^{x} \times T^{v} \in \mathcal{T}_{h}$ and prove the corresponding estimate there. Let $\partial T^{v}=\gamma_{1}^{ \pm} \cup \gamma_{2}^{ \pm}$with $\gamma_{i}^{ \pm}$denoting the edges of $\partial T^{v}$ in the $v_{i}$-direction defined as in (106). Adding and subtracting $\mathcal{P}_{v}^{0}(\mathbf{E})$ we can decompose $\mathcal{K}^{2}$ as

$$
\mathcal{K}_{R}^{2}\left(\mathbf{E}_{h}, f, \omega^{h}\right)=\mathcal{K}_{R}^{2}\left(\mathbf{E}_{h}-\mathcal{P}^{0}(\mathbf{E}), f, \omega^{h}\right)+\mathcal{K}_{R}^{2}\left(\mathcal{P}^{0}(\mathbf{E}), f, \omega^{h}\right) .
$$

For the first term, Hölder inequality together with trace and inverse inequalities and estimate (41) give,

$$
\begin{aligned}
\left|\mathcal{K}_{R}^{2}\left(\mathbf{E}_{h}-\mathcal{P}^{0}(\mathbf{E}), f, \omega^{h}\right)\right| & \leq\left\|\mathbf{E}_{h}-\mathcal{P}^{0}(\mathbf{E})\right\|_{0, \infty, T^{x}} \\
& \times\left(\left\|\omega^{e}\right\|_{0, R}\left\|\nabla_{v} \omega^{h}\right\|_{0, R}+\sum_{i=1,2}\left\|\omega^{e}\right\|_{0, T^{x} \times \gamma_{i}^{ \pm}}\left\|\omega^{h}\right\|_{0, T^{x} \times \gamma_{i}^{ \pm}}\right) \\
& \leq C\left\|\mathbf{E}_{h}-\mathcal{P}^{0}(\mathbf{E})\right\|_{0, \infty, T^{x}} h^{k}\|f\|_{k+1, R}\left\|\omega^{h}\right\|_{0, R} .
\end{aligned}
$$

Writing now $\mathbf{E}_{h}-\mathcal{P}^{0}(\mathbf{E})=\mathbf{E}_{h}-\mathbf{E}+\mathbf{E}-\mathcal{P}^{0}(\mathbf{E})$ and using triangle inequality together with the $L^{\infty}$ estimate (12) we finally get

$\left|\mathcal{K}_{R}^{2}\left(\mathbf{E}_{h}-\mathcal{P}^{0}(\mathbf{E}), f, \omega^{h}\right)\right| \leq\left(C h^{k}\left\|\mathbf{E}-\mathbf{E}_{h}\right\|_{0, \infty, T^{x}}+C h^{k+1}\|\mathbf{E}\|_{1, \infty, T^{x}}\right)\|f\|_{k+1, R}\left\|\omega^{h}\right\|_{0, R}$.

To conclude we need to estimate last term in (115). However, we need to distinguish several cases according to whether $\mathbf{E}$ or any of its components vanishes inside $R$ :

(i) : None of the components of $\mathbf{E}$ vanish inside $R$,

(ii) : both components $E_{1}$ and $E_{2}$ vanish at some point: $\exists \mathbf{x}^{*} \in T^{x}$ such that $\mathbf{E}\left(\mathbf{x}^{*}\right)=\mathbf{0}$,

(iii) : only one of the components vanishes, say $E_{1}$; i.e. $\exists \mathrm{x}^{*} \in T^{x}$ s.t. $E_{1}\left(\mathbf{x}^{*}\right)=0$ but $E_{2}(\mathbf{x}) \neq 0 \forall \mathbf{x} \in T^{x}$.

In the first case (i), estimate (108) from Lemma 12 provides the desired bound.

In the case (ii), $\Pi_{h}=\widetilde{\Pi}_{x} \otimes \mathcal{P}_{v, 1} \otimes \mathcal{P}_{v, 2}$. Then, Hölder inequality, estimates (41) together with inverse and trace inequalities and the stability in $L^{\infty}$ of the $L^{2}$ projection (11), give

$$
\begin{aligned}
\left|\mathcal{K}_{R}^{2}\left(\mathcal{P}^{0}(\mathbf{E}), f, \omega^{h}\right)\right| \leq & \left\|\mathcal{P}^{0}(\mathbf{E})\right\|_{0, \infty, T^{x}} \\
& \times\left(\left\|\omega^{e}\right\|_{0, R}\left\|\nabla_{v} \omega^{h}\right\|_{0, R}+\sum_{i=1,2}\left\|\omega^{e}\right\|_{0, T^{x} \times \gamma_{i}^{ \pm}}\left\|\omega^{h}\right\|_{0, T^{x} \times \gamma_{i}^{ \pm}}\right) \\
\leq & C\|\mathbf{E}\|_{0, \infty, T^{x}} h^{k}\|f\|_{k+1, R}\left\|\omega^{h}\right\|_{0, R} .
\end{aligned}
$$

Using now the fact that $\exists \mathbf{x}^{*} \in T^{x}$ such that $\mathbf{E}\left(\mathbf{x}^{*}\right)=\mathbf{0}$ together with the mean value theorem and the assumed regularity of $\mathbf{E}$, we find

$$
\|\mathbf{E}\|_{0, \infty, T^{x}}=\max _{\mathbf{x} \in T^{x}}\left|\mathbf{E}(\mathbf{x})-\mathbf{E}\left(\mathbf{x}^{*}\right)\right| \leq C \max _{\mathbf{x} \in T^{x}}\left|\mathbf{x}-\mathbf{x}^{*}\right||\mathbf{E}|_{1, \infty, T^{x}} \leq C h_{T^{x}}|\mathbf{E}|_{1, \infty, T^{x}} .
$$


Hence, in case (ii) we have

$$
\left|\mathcal{K}_{R}^{2}\left(\mathcal{P}^{0}(\mathbf{E}), f, \omega^{h}\right)\right| \leq C h^{k+1}|\mathbf{E}|_{1, \infty, T^{x}}\|f\|_{k+1, R}\left\|\omega^{h}\right\|_{0, R} .
$$

Finally, we deal with the last case (iii). Without loss of generality we assume that $E_{1}>0$ on $R$ and $E_{2}$ vanishes at some $\mathbf{x}^{*} \in T^{x}$. Arguing as for the splitting in (110) we can write

$$
\mathcal{K}_{R}^{2}\left(\mathcal{P}^{0}(\mathbf{E}), f, \varphi\right)=\mathcal{K}_{R}^{v_{1}}\left(\mathcal{P}^{0}(\mathbf{E}), f, \varphi\right)+\mathcal{K}_{R}^{v_{2}}\left(\mathcal{P}^{0}(\mathbf{E}), f, \varphi\right)
$$

where the terms $\mathcal{K}_{R}^{v_{1}}$ and $\mathcal{K}_{R}^{v_{2}}$ defined as in (111). Now, since $E_{2}$ vanishes inside $R$ but $E_{1}$ does not, estimate (109) from Lemma 12 gives the bound for $\mathcal{K}_{R}^{v_{1}}$. Hence to conclude we only need to estimate the term $\mathcal{K}_{R}^{v_{2}}\left(\mathcal{P}^{0}(\mathbf{E}), f, \varphi\right)$. Reasoning as for the case (i); the fact that $\exists \mathbf{x}^{*} \in T^{x}$ such that $E_{2}\left(\mathbf{x}^{*}\right)=0$ together with the mean value theorem and the assumed regularity of $\mathbf{E}$, gives

$$
\left\|E_{2}\right\|_{0, \infty, T^{x}}=\max _{\mathbf{x} \in T^{x}}\left|E_{2}(\mathbf{x})-E_{2}\left(\mathbf{x}^{*}\right)\right| \leq C \max _{\mathbf{x} \in T^{x}}\left|\mathbf{x}-\mathbf{x}^{*}\right|\left|E_{2}\right|_{1, \infty, T^{x}} \leq C h_{T^{x}}|\mathbf{E}|_{1, \infty, T^{x}}
$$

and so using this bound together with the $L^{\infty}$ of the $L^{2}$-projection (11), Hölder inequality, estimates (41) and trace and inverse inequalities, we finally get

$$
\begin{aligned}
\left|\mathcal{K}_{R}^{v_{2}}\left(\mathcal{P}^{0}(\mathbf{E}), f, \omega^{h}\right)\right| \leq & \left\|\mathcal{P}_{v, 2}^{0}\left(E_{2}\right)\right\|_{0, \infty, T^{x}} \\
& \times\left(\left\|\omega^{e}\right\|_{0, R} h^{-1}\left\|\omega^{h}\right\|_{0, R}+\sum_{i=1,2}\left\|\omega^{e}\right\|_{0, T^{x} \times \gamma_{i}^{ \pm}}\left\|\omega^{h}\right\|_{0, T^{x} \times \gamma_{i}^{ \pm}}\right) \\
\leq & C\left\|E_{2}\right\|_{0, \infty, T^{x}} h^{k}\|f\|_{k+1, R}\left\|\omega^{h}\right\|_{0, R} \\
\leq & C h^{k+1}|\mathbf{E}|_{1, \infty}\|f\|_{k+1, R}\left\|\omega^{h}\right\|_{0, R},
\end{aligned}
$$

which together with estimate (109) give the desired estimate also in the case (iii). Summing over all elements of the partition, the above estimate together with estimates (116), (117) and (108) from Lemma 12 we reach (49) and conclude the proof of the lemma.

Acknowledgements. BA is grateful to Daniele Boffi from Università degli Studi di Pavia and to Jesús García-Azorero from Universidad Autónoma of Madrid, for helpful and fruitful discussions while carrying out this work. She also thanks Jonnhy Guzmán from Brown University for his careful reading of parts of Appendix A.4. BA also acknowledge the he kind hospitality from the Division of Applied Mathematics at Brown while she was visiting in Spring 2010. BA has been partially supported by Ministerio de Ciencia e Innovación under projects MTM2008-03541and HI2008-0173. JAC was supported by the projects Ministerio de Ciencia e Innovación MTM2008-06349-C03-03 and 2009-SGR-345 from Agència de Gestió d'Ajuts Universitaris i de Recerca-Generalitat de Catalunya. CWS is partially supported by National Science Fundation grant DMS-0809086 and Department of Energy grant DE-FG02-08ER25863 


\section{REFERENCES}

[1] G. Acosta And R. G. Durán, An optimal Poincaré inequality in $L^{1}$ for convex domains, Proc. Amer. Math. Soc., 132(1) (2004), 195-202 (electronic).

[2] R. A. Adams, Sobolev Spaces, Pure and Applied Mathematics, vol. 65, Academic Press [A subsidiary of Harcourt Brace Jovanovich, Publishers], New York-London, 1975.

[3] S. Agmon, Lectures on Elliptic Boundary Value Problems, Prepared for publication by B. Frank Jones, Jr. with the assistance of George W. Batten, Jr., Van Nostrand Mathematical Studies, no. 2, D. Van Nostrand Co., Inc., Princeton, N.J.-Toronto-London, 1965.

[4] D. N. ARnold, An interior penalty finite element method with discontinuous elements, SIAM J. Numer. Anal., 19(4) (1982), 742-760.

[5] D. N. Arnold, F. Brezzi, B. Cockburn, And L. D. Marini, Unified analysis of discontinuous Galerkin methods for elliptic problems, SIAM J. Numer. Anal., 39(5) (2001/02), 1749-1779 (electronic).

[6] B. Ayuso, J. A. Carrillo, And C.-W. Shu, Discontinuous Galerkin methods for the One-Dimensional Vlasov-Poisson system, submitted, 2009.

[7] B. Ayuso, J. A. Carrillo, And C.-W. Shu, Discontinuous Galerkin methods for the One-Dimensional Vlasov-Poisson system, Technical report, Brown University, 2009. http://www. dam. brown.edu/scicomp/reports/2009-41/

[8] B. Ayuso And L. D. MARInI, Discontinuous Galerkin methods for advection-diffusionreaction problems, SIAM J. Numer. Anal., 47(2) (2009), 1391-1420.

[9] J. BAtt AND G. REIn, Global classical solutions of the periodic Vlasov-Poisson system in three dimensions, C. R. Acad. Sci. Paris Sér. I Math., 313(6) (1991), 411-416.

[10] F. Bouchut, F. Golse, And M. Pulvirenti, Kinetic Equations and Asymptotic Theory, B. Perthame and L. Desvillettes, eds., Series in Applied Mathematics (Paris), vol. 4, Gauthier-Villars, Éditions Scientifiques et Médicales Elsevier, Paris, 2000.

[11] S. C. Brenner, Poincaré-Friedrichs inequalities for piecewise $H^{1}$ functions, SIAM J. Numer. Anal., 41(1) (2003), 306-324 (electronic).

[12] F. Brezzi, J. Douglas, JR., M. Fortin, And L. D. Marini, Efficient rectangular mixed finite elements in two and three space variables, RAIRO Modél. Math. Anal. Numér., 21(4) (1987), 581-604.

[13] F. Brezzi, J. Douglas, JR., And L. D. Marini, Two families of mixed finite elements for second order elliptic problems, Numer. Math., 47(2) (195), 217-235.

[14] F. Brezzi, J. Douglas, JR., And L. D. Marini., Variable degree mixed methods for second order elliptic problems, Mat. Apl. Comput., 4(1) (1985), 19-34.

[15] F. Brezzi And M. Fortin, Mixed and Hybrid Finite Element Methods, Springer Series in Computational Mathematics, vol. 15, Springer-Verlag, New York, 1991.

[16] F. Brezzi, L. D. MARInI, AND E. SüLI, Discontinuous Galerkin methods for first-order hyperbolic problems, Math. Models Methods Appl. Sci., 14(12) (2004), 1893-1903.

[17] A. Buffa And C. ORTner, Compact embeddings of broken Sobolev spaces and applications, IMA J. Numer. Anal., 29(4) (2009), 827-855.

[18] P. Castillo, B. Cockburn, I. Perugia, and D. Schötzau, An a priori error analysis of the local discontinuous Galerkin method for elliptic problems, SIAM J. Numer. Anal., 38(5) (2000), 1676-1706 (electronic).

[19] H. CHEn, Pointwise error estimates of the local discontinuous Galerkin method for a second order elliptic problem, Math. Comp., 74(251) (2005), 1097-1116 (electronic).

[20] P. G. Ciarlet, Basic error estimates for elliptic problems, in Handbook of Numerical Analysis, Vol. II,, North-Holland, Amsterdam, 1991, 17-351. 
[21] B. Cockburn, G. Kanschat, I. Perugia, and D. Schötzau, Superconvergence of the local discontinuous Galerkin method for elliptic problems on Cartesian grids, SIAM J. Numer. Anal., 39(1) (2001), 264-285 (electronic).

[22] M. Crouzeix and V. ThoméE, The stability in $L_{p}$ and $W_{p}^{1}$ of the $L_{2}$-projection onto finite element function spaces, Math. Comp., 48(178) (1987), 521-532.

[23] E. Dari, R. G. Durán, And C. PAdra, Maximum norm error estimators for threedimensional elliptic problems, SIAM J. Numer. Anal., 37(2) (2000), 683-700 (electronic).

[24] M. Dauge, Elliptic Boundary Value Problems on Corner Domains, Smoothness and asymptotics of solutions, Lecture Notes in Mathematics, vol 1341, Springer-Verlag, Berlin, 1988.

[25] M. Dauge, Problèmes de Neumann et de Dirichlet sur un polyèdre dans $\mathbb{R}^{3}$ : régularité dans des espaces de Sobolev $L_{p}$, C. R. Acad. Sci. Paris Sér. I Math., 307(1) (1988), 27-32.

[26] J. Dolbeault, An introduction to kinetic equations: the Vlasov-Poisson system and the Boltzmann equation, Current developments in partial differential equations (Temuco, 1999), Discrete Contin. Dyn. Syst., 8(2) (2002), 361-380.

[27] L. Gastaldi and R. Nochetto, Optimal $L^{\infty}$-error estimates for nonconforming and mixed finite element methods of lowest order, Numer. Math., 50(5) (1987), 587-611.

[28] L. Gastaldi And R. H. Nochetto, Sharp maximum norm error estimates for general mixed finite element approximations to second order elliptic equations, RAIRO Modél. Math. Anal. Numér., 23(1) (1989), pp. 103-128.

[29] D. Gilbarg and N. S. Trudinger, Elliptic Partial Differential Equations of Second Order, Reprint of the 1998 edition, Classics in Mathematics, Springer-Verlag, Berlin, 2001.

[30] R. T. Glassey, The Cauchy Problem in Kinetic Theory, Society for Industrial and Applied Mathematics (SIAM), Philadelphia, PA, 1996.

[31] J. Guzmán, D. Leykekhman, J. Rossmann, and A. H. Schatz, Hölder estimates for Green's functions on convex polyhedral domains and their applications to finite element methods, Numer. Math., 112(2) (2009), 221-243.

[32] D. Henry, Geometric Theory of Semilinear Parabolic Equations, Lecture Notes in Mathematics, vol. 840, Springer-Verlag, Berlin, 1981.

[33] P. Lasaint And P.-A. Raviart, On a finite element method for solving the neutron transport equation, in Mathematical Aspects of Finite Elements in Partial Differential Equations (Proc. Sympos., Math. Res. Center, Univ. Wisconsin, Madison, Wis., 1974), publication no. 33, Math. Res. Center, Univ. of Wisconsin-Madison, Academic Press, New York, 1974, 89-123.

[34] F. NATtERER, Über die punktweise Konvergenz finiter Elemente, Numer. Math., 25(1) $(1975 / 76), 67-77$.

[35] J. Nitsche, $L_{\infty}$-convergence of finite element approximations, in Mathematical Aspects of Finite Element Methods (Proc. Conf., Consiglio Naz. delle Ricerche (C.N.R.), Rome, 1975), Lecture Notes in Math., vol. 606. Springer, Berlin, 1977, 261-274.

[36] J. A. Nitsche, $L_{\infty}$-convergence of finite element approximation, in Journées "Éléments Finis" (Rennes, 1975), Univ. Rennes, Rennes, 1975, 18.

[37] A. H. Schatz, Pointwise error estimates and asymptotic error expansion inequalities for the finite element method on irregular grids. I. Global estimates, Math. Comp., 67(223) (1998), 877-899.

[38] A. H. Schatz AND L. B. WahlBin, Interior maximum-norm estimates for finite element methods. II, Math. Comp., 64(211) (1995), 907-928. 
[39] D. Schötzau And C. Schwab, Time discretization of parabolic problems by the hpversion of the discontinuous Galerkin finite element method, SIAM J. Numer. Anal., 38(3) (2000), 837-875 (electronic).

[40] Q. ZHANG AND C.-W. SHU, Error estimates to smooth solutions of Runge-Kutta discontinuous Galerkin methods for scalar conservation laws, SIAM J. Numer. Anal., $42(2$ (2004), 641-666 (electronic).

Blanca Ayuso de Dios

Centre de Recerca Matemàtica (CRM)

08193 Bellaterra, Barcelona, Spain

E-mail address: bayuso@crm.cat

José A. CARrillo

ICREA AND DEPARTAMENT DE MATEMÀtiqueS

Universitat Autònoma de Barcelona

E-08193 BellaterRA, SpAin

E-mail address: carrillo@mat.uab.es

Chi-Wang Shu

Division of Applied Mathematics, Brown

University, Providence RI 16802, USA

E-mail address: shu@dam.brown.edu 